\title{
2010
}

Nevada National Security Site Annual IIIness and Injury Surveillance Report

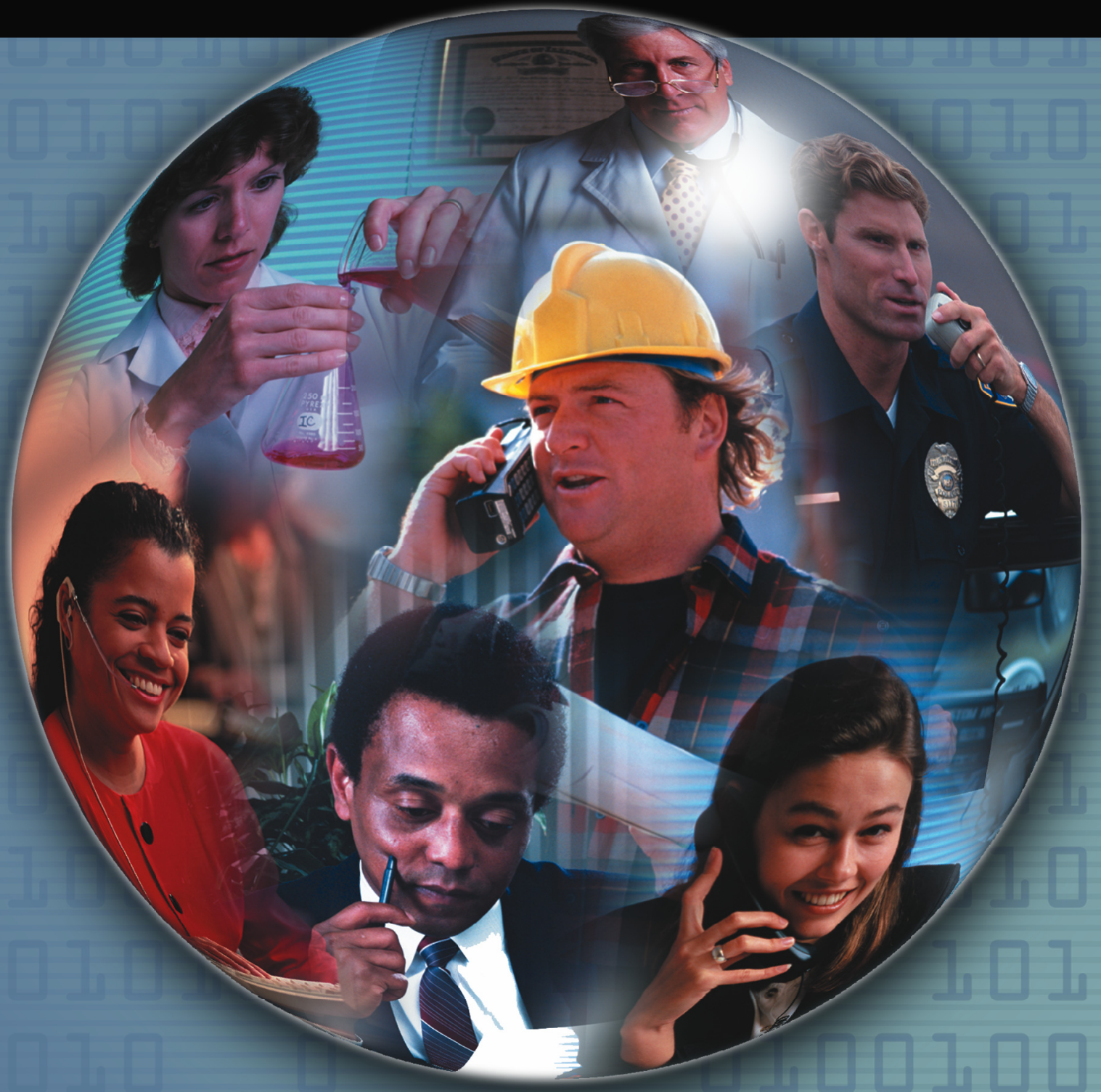




\section{Nevada National Security Site 2010 Illness and Injury Surveillance Report}

Questions or comments about this report or the Illness and Injury Surveillance Program (IISP) may be directed to:

E-mail:

Dr. Cliff Strader at cliff.strader@hq.doe.gov or Dr. Bonnie Richter at bonnie.richter@hq.doe.gov

or direct letters to:

Mail Stop HS-13 / GTN Building

U.S. Department of Energy

1000 Independence Avenue, S.W.

Washington, DC 20585-0270

Additional information about the Department of Energy's Office of Illness and Injury Prevention Programs, the IISP, and annual reports for DOE sites participating in this program can be found at:

http://www.hss.energy.gov/healthsafety/WSHP/epi/surv/

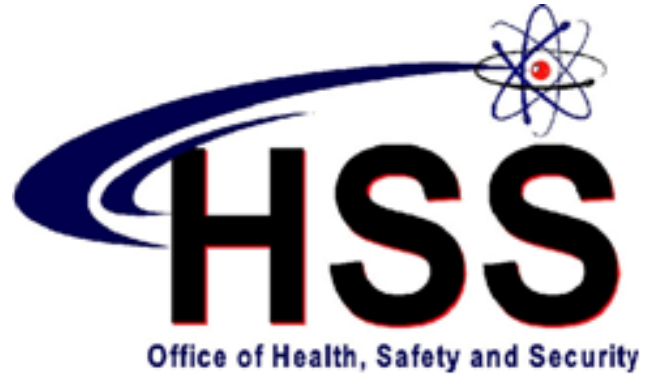

ACKNOWLEDGEMENT

LifeART images copyright 2000 Lippincott Williams \& Wilkins. All rights reserved.

This document was produced under contract number DE-AC05-06OR23100 between the

U.S. Department of Energy and Oak Ridge Associated Universities. 


\section{Nevada National Security Site \\ 2010 Illness and Injury Surveillance Report \\ At A Glance}

NOTE: In previous years, the number of days absent or lost and the age of the work force were reported as averages. Beginning in 2010, they are being reported as medians. A median is the value in a population that half the values exceed and half fall short of it. The median is less affected than the average when the distribution contains a few very large or very small values.

A total of 3,935 NNSS employees were included in illness and injury surveillance in 2010, a decrease of 14 percent since 2009. There were 1,016 (26 percent) women and 2,919 (74 percent) men in the work force. The median age was 50 years for women and 51 years for men, unchanged from 2009.

The 135 absences reported by 110 women resulted in an absence rate of 13 absences per 100 workers $(135 / 1,016)$. Among the 2,919 men, 223 absences resulted in an absence rate of 8 absences per 100 workers $(223 / 2,919)$. The median length of absence among women was 13 days, compared with 8 days for men. The median length of absence for men has steadily decreased from 11 days in 2006 to 8 days in 2010. Among women, the median length of absence has varied without consistent change since 2006.

Security and Fire workers had the highest absence rate among both men and women, 15 absences per 100 men (47/304) and 28 absences per 100 women $(5 / 18)$.

Women lost 2,892 calendar days due to illness and injury in 2010.

Musculoskeletal disorders (17 percent), unspecified symptoms (predominantly symptoms of digestion, fatigue, and chest pain) (16 percent), and injuries (12 percent) accounted for 45 percent of all reported diagnoses among women. 
Men lost 5,076 calendar days due to illness and injury. Fifty-eight percent of all reported diagnoses among men were due to injuries ( 21 percent), unspecified symptoms (primarily symptoms of chest pain, digestion, dizziness, and headache) (21 percent), and musculoskeletal disorders (16 percent).

The age-adjusted rates of musculoskeletal diagnoses showed a general decline among men and women from 2006 to 2010. This decline resulted from a decrease in all types of musculoskeletal conditions.

One definite and eight possible sentinel health events for occupational (SHEO) diagnoses were reported in 2010. Five diagnoses were for carpal tunnel syndrome. The SHEOs resulted in a total of 183 days absent.

Men reported 38 and women reported 13 OSHA-recordable events in 2010. The rate of OSHA events was approximately 1 event per 100 workers among both men and women. For women, there has been no consistent change in the rate of OSHA events since 2006; for men, the rate has decreased from 2 events per 100 workers in 2006 to 1 event per 100 workers for the remaining years.

Occupational illnesses and injuries reported at NNSS in 2010 resulted in 985 lost or restricted workdays, a 35 percent increase since 2009. Technical Support workers had the highest rate of OSHA events among men and women (slightly less than 4 events per 100 workers). Men in the Technical Support group showed an increase in musculoskeletal conditions and injuries and women reported an increase in injuries compared with 2009.

Among Security and Fire workers, the rate of OSHA-recordable diagnoses declined from 77 diagnoses per 1,000 workers among women and 121 diagnoses per 1,000 workers among men in 2006 to 0 diagnoses in 2010; during this time period, the majority of diagnoses were musculoskeletal disorders and injuries among both men and women.

Injuries accounted for 64 percent of the OSHA diagnoses for women and 67 percent of the diagnoses among men in 2010. The most common injuries reported were sprains and strains for men and unspecified injuries for women. Since 2006, injuries have accounted for at least 50 percent of the OSHA 
diagnoses for both women and men, with sprains and strains being among the most common diagnoses each year.

The type of accident most commonly reported for OSHA events among men was overexertion and strenuous movements and falls among women. 


\section{The Nevada National Security Site Work Force - 2010}

The Work Force by Gender and Age 1

The Work Force by Gender and Job

Category 1

Number and Length of Absences

Absence Rate by Gender and Age 2

Number of Days Absent by

Gender and Age

Absence Rate by Job Category

and Gender 3

Median Duration of Absence by

Job Category and Gender. ... 3

\section{Diagnostic Categories}

Number of Diagnoses and Lost Calendar Days by Diagnostic Category

(Categorized by ICD-9-CM) and Gender

Common Diagnoses Among Female

Workers in 2010

Common Diagnoses Among Male

Workers in 2010 .. 6

Number of Most Frequently Reported Diagnoses by Job Category and Gender 7

\section{Rates of Disease Occurrence}

Rates for All Illnesses and Injuries Combined by Job Category, Gender, and Age

Rates for Selected Diagnostic Categories by Job Category, Gender, and Age .8

\section{Time Trends}

Age-Adjusted Rates for All Diagnoses Combined Among Women and Men from 2006 to 2010
Age-Adjusted Rates for Selected Diagnostic Categories Among Women and Men from 2006 to 2010

Age-Adjusted Rates for All Diagnoses

Combined Among Women and Men by Job

Category from 2006 to $2010 \ldots \ldots \ldots \ldots \ldots \ldots \ldots \ldots . \ldots 12$

\section{Sentinel Health Events for Occupations (SHEOs)}

Characteristics of SHEOs by Gender 13

SHEO Diagnoses by Gender 13

Occupational Safety and Health Administration (OSHA)-Recordable Events

OSHA-Recordable Events by Gender and Age. 14

OSHA-Recordable Events by Job

Category and Gender 14

\section{Diagnostic and Accident Categories for OSHA-Recordable Events}

OSHA-Recordable Diagnoses by

Diagnostic Category and Gender

OSHA-Recordable Accidents by Type

and Gender

Rates of OSHA-Recordable Events

OSHA-Recordable Rates by Age and Job

Categories Among Women, All Diagnoses

Combined

OSHA-Recordable Rates by Age and Job

Categories Among Men, All Diagnoses

Combined

Time Trends for OSHA-Recordable Events

Age-Adjusted Rates for All OSHA-Recordable Diagnoses Combined Among Women and Men by Job Category from 2006 to 2010 .

\section{Appendices}


The Nevada National Security Site Work Force - 2010

Figure 1. The Work Force by Gender and Age

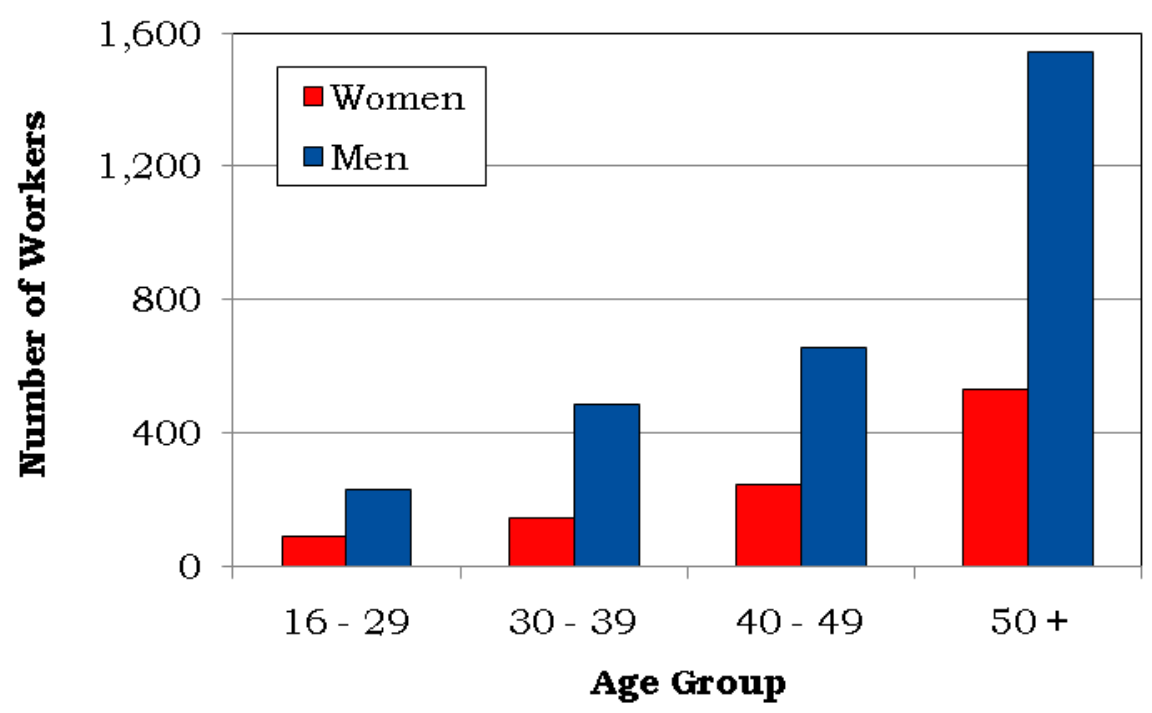

Figure 2. The Work Force by Gender and Job Category

\begin{tabular}{|l|c|c|}
\hline \multicolumn{1}{|c|}{ Job Category } & W omen & M en \\
\hline \multirow{2}{*}{ Professional } & 568 & 1,351 \\
& $56 \%$ & $46 \%$ \\
\hline \multirow{2}{*}{ Administrative Support } & 212 & 42 \\
& $21 \%$ & $1 \%$ \\
\hline \multirow{2}{*}{ Technical Support } & 135 & 426 \\
& $13 \%$ & $15 \%$ \\
\hline \multirow{2}{*}{ Service } & 25 & 80 \\
& $2 \%$ & $3 \%$ \\
\hline \multirow{2}{*}{ Crafts } & 18 & 304 \\
& $2 \%$ & $10 \%$ \\
\hline \multirow{2}{*}{ Line Operators } & 58 & 715 \\
\hline \multirow{2}{*}{ Total } & $6 \%$ & $24 \%$ \\
\hline
\end{tabular}




\section{Number and Length of Absences}

Figure 3. Absence Rate by Gender and Age

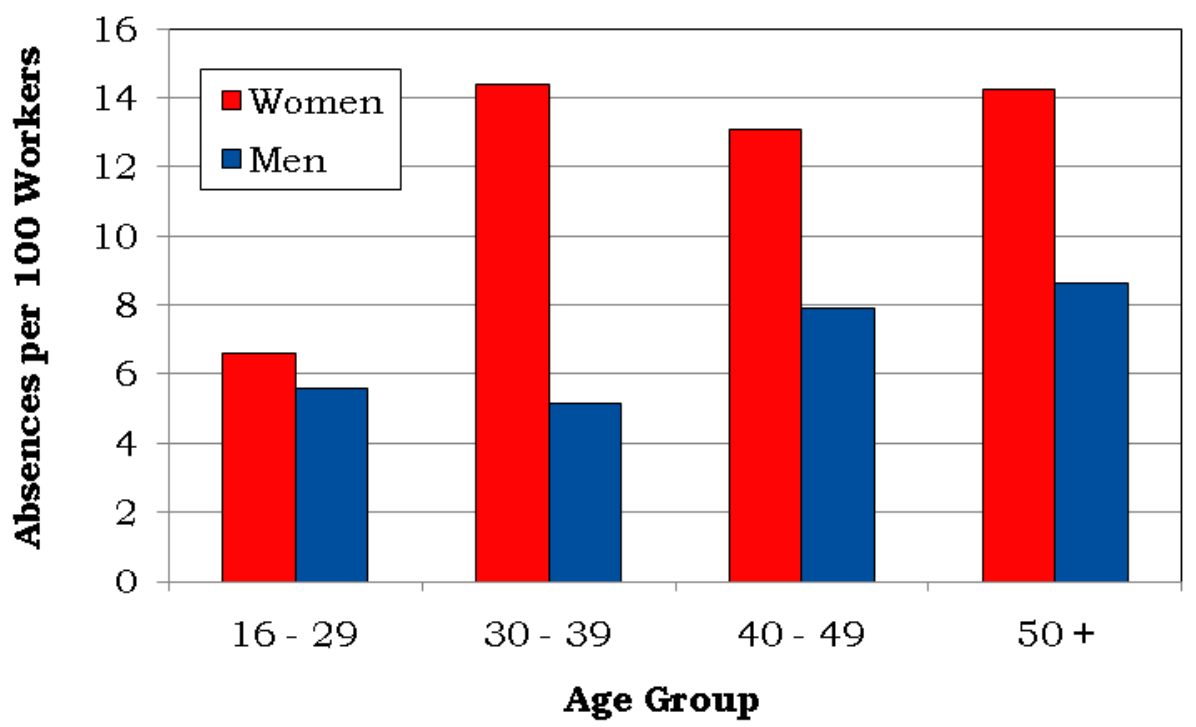

Figure 4. Number of Days Absent by Gender and Age

\begin{tabular}{|c|c|c|c|c|}
\hline \multirow{3}{*}{ Gender } & \multirow{2}{*}{ Age } & \multirow{2}{*}{$\begin{array}{c}\text { Number of } \\
\text { Absences }\end{array}$} & \multicolumn{2}{|c|}{ Number of Days Absent } \\
\cline { 3 - 5 } & & 6 & Total & M edian \\
\hline \multirow{4}{*}{ Women* } & $16-29$ & 62 & 32 & 3 \\
\cline { 2 - 5 } & $30-39$ & 21 & 244 & 7 \\
\cline { 2 - 5 } & $40-49$ & 32 & 641 & 13 \\
\cline { 2 - 5 } & $50+$ & 76 & 1,975 & 15 \\
\cline { 2 - 5 } & Total & 135 & 2,892 & 13 \\
\hline \multirow{4}{*}{ Men } & $16-29$ & 13 & 129 & 5 \\
\cline { 2 - 5 } & $30-39$ & 25 & 530 & 11 \\
\cline { 2 - 5 } & $40-49$ & 52 & 1,599 & 7 \\
\cline { 2 - 5 } & $50+$ & 133 & 2,818 & 12 \\
\cline { 2 - 5 } & Total & 223 & 5,076 & 8 \\
\hline
\end{tabular}

*Normal pregnancies were excluded from absences for women. 
Figure 5. Absence Rate by Job Category and Gender

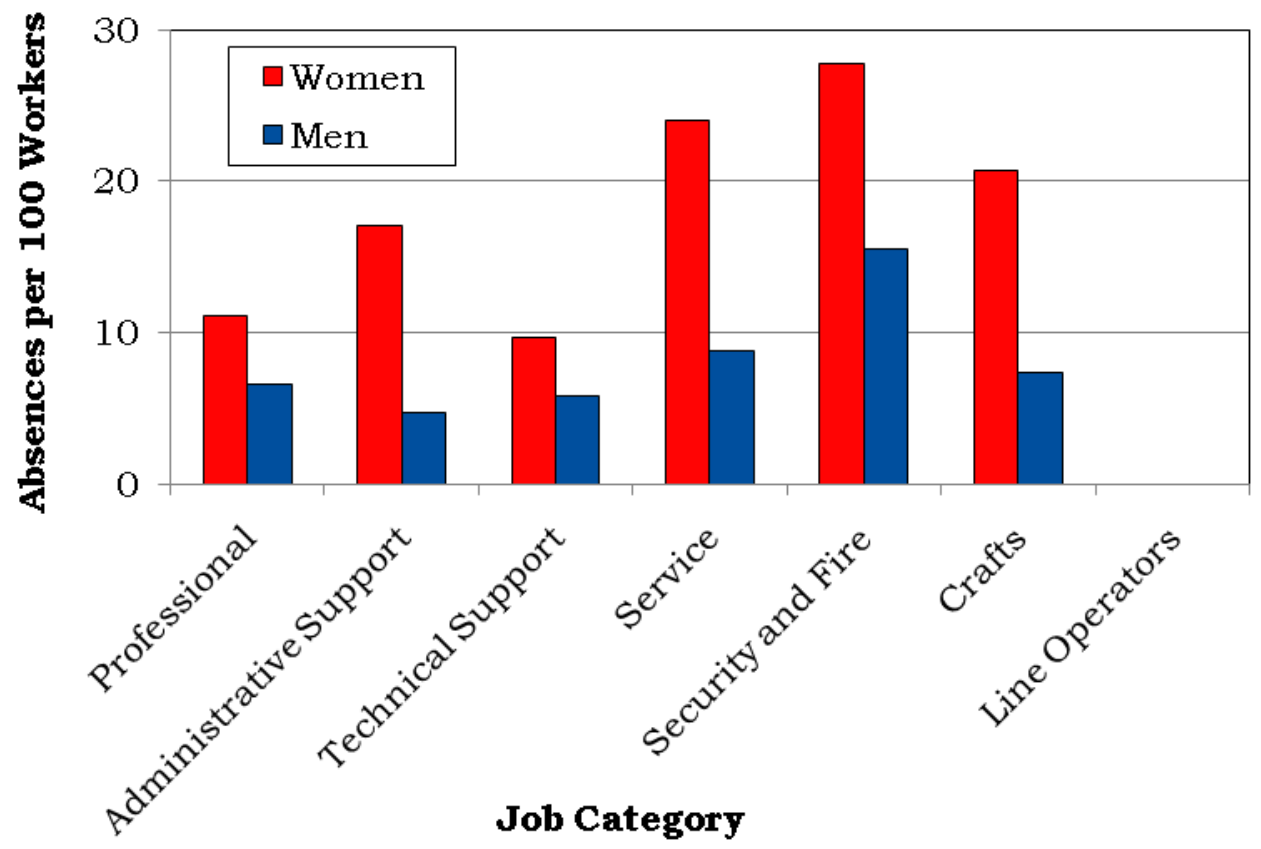

Figure 6. Median Duration of Absence by Job Category and Gender

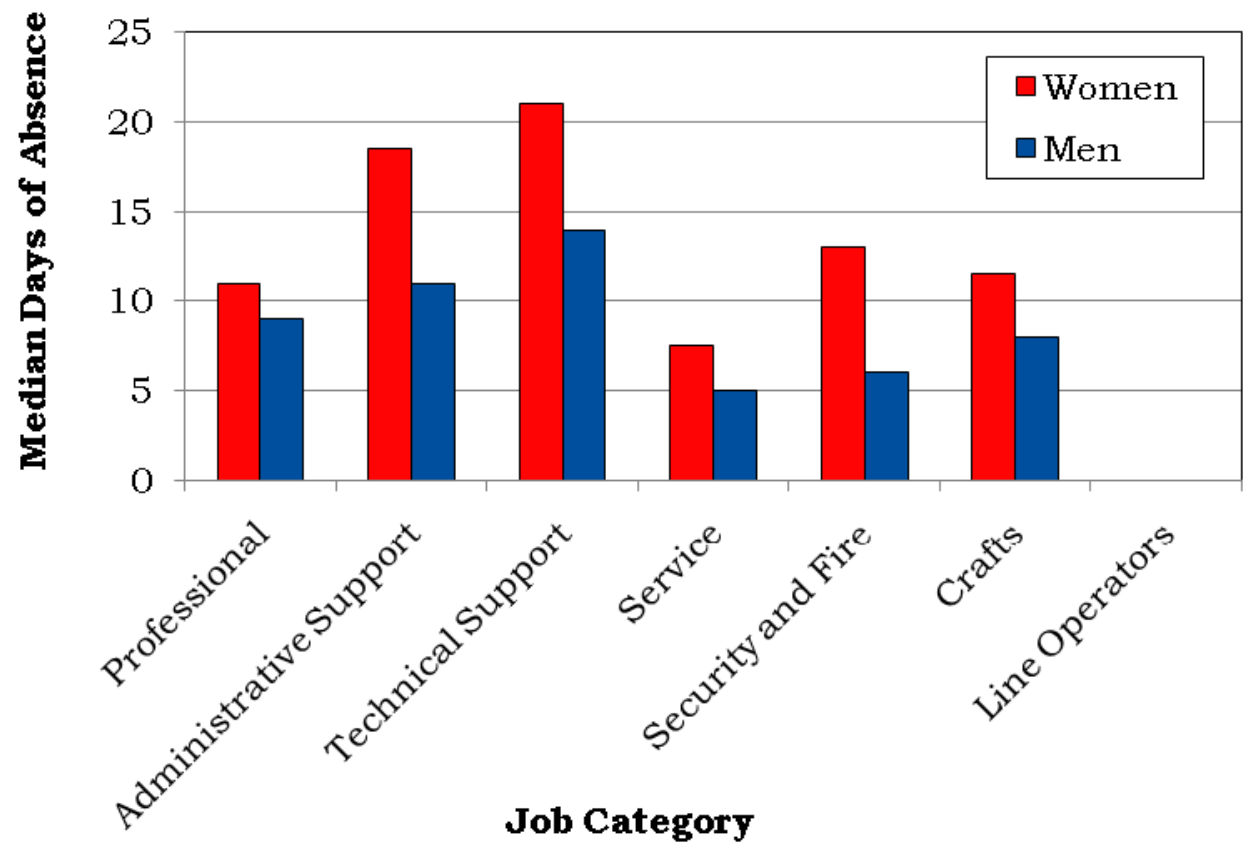




\section{Diagnostic Categories}

Figure 7. Number of Diagnoses and Lost Calendar Days by Diagnostic Category (Categorized by ICD-9-CM) and Gender

\begin{tabular}{|c|c|c|c|c|}
\hline \multirow[b]{2}{*}{ Diagnostic Category } & \multicolumn{2}{|c|}{ Women } & \multicolumn{2}{|c|}{ Men } \\
\hline & $\begin{array}{l}\text { Number of } \\
\text { Diagnoses }\end{array}$ & $\begin{array}{c}\text { Number } \\
\text { of Lost } \\
\text { Calendar } \\
\text { Days }\end{array}$ & $\begin{array}{l}\text { Number of } \\
\text { Diagnoses }\end{array}$ & $\begin{array}{c}\text { Number } \\
\text { of Lost } \\
\text { Calendar } \\
\text { Days }\end{array}$ \\
\hline Benign Growths & 5 & 181 & 2 & 76 \\
\hline Blood & $\mathbf{0}$ & 0 & 6 & 67 \\
\hline Cancer & 4 & 116 & 7 & 279 \\
\hline Digestive & 25 & 239 & 32 & 422 \\
\hline Endocrine / Me tabolic & 13 & 147 & 12 & 285 \\
\hline Existing Birth Condition & 0 & 0 & 2 & 138 \\
\hline Genitourinary & 24 & 319 & 12 & 80 \\
\hline Heart/Circulatory & 14 & 445 & 42 & 723 \\
\hline Infections/Parasites & 10 & 128 & 12 & 109 \\
\hline Injury & 32 & 688 & 91 & 2,122 \\
\hline Miscarriage & 1 & 21 & NA & NA \\
\hline Musculoskeletal & 44 & 1,034 & 71 & 1,660 \\
\hline Nervous System & 14 & 263 & 13 & 266 \\
\hline Psychological & 5 & 37 & 2 & 9 \\
\hline Respiratory & 29 & 186 & 36 & 184 \\
\hline Skin & 2 & 16 & 9 & 113 \\
\hline Unspecified Symptoms & 41 & 518 & 91 & 630 \\
\hline
\end{tabular}

Note: Lost calendar days for each absence are counted more than once when multiple diagnoses occur in different diagnostic categories for the same absence. 


\section{Figure 8. Common Diagnoses Among Female Workers in 2010}

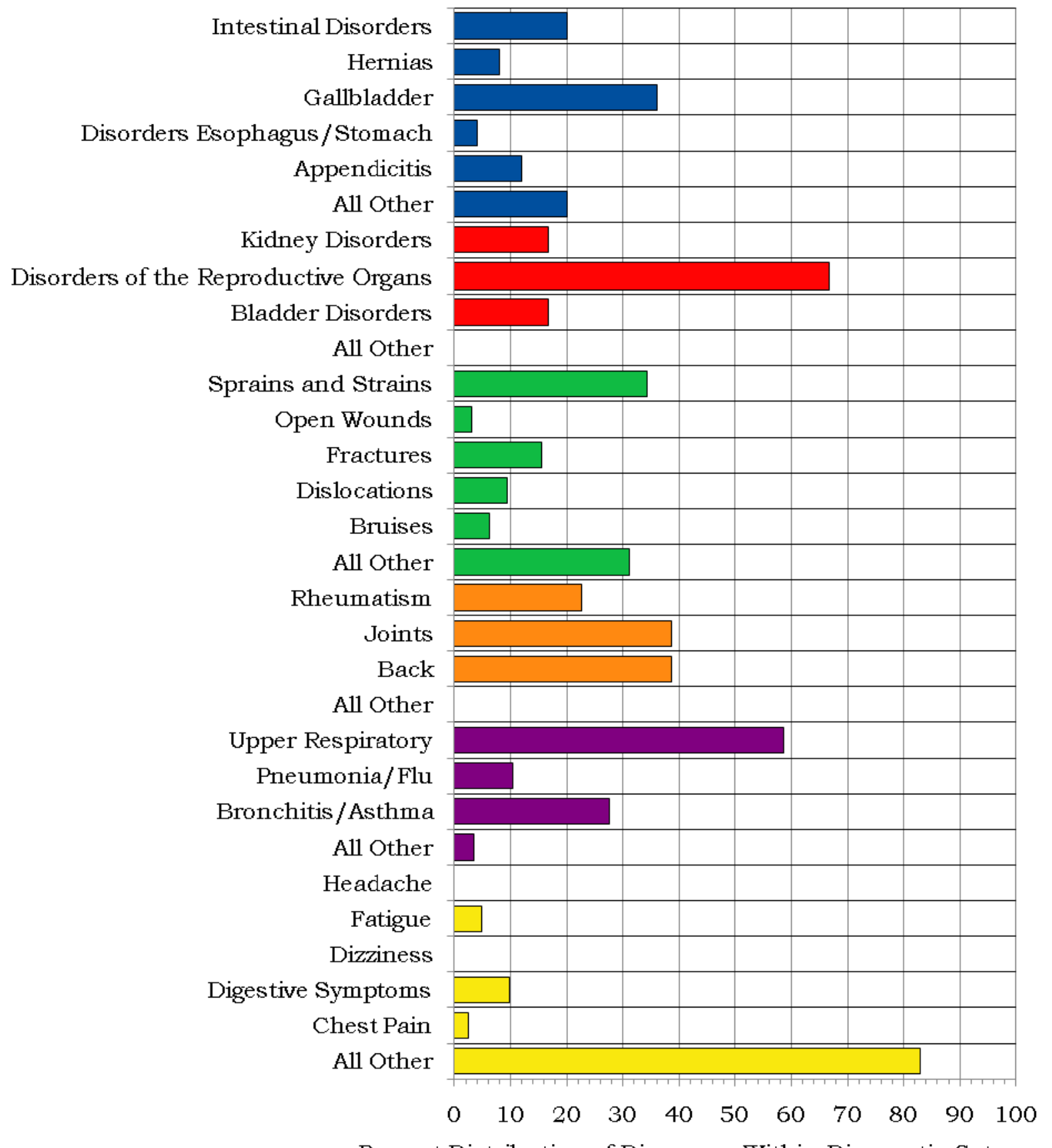

Percent Distribution of Diagnoses Within Diagnostic Category

Digestive, 25 Diagnoses

Genitourinary, 24 Diagnoses

Injury, 32 Diagnoses
Musculoskeletal, 44 Diagnoses

Respiratory, 29 Diagnoses

Unspecified Symptoms, 41 Diagnoses 


\section{Figure 9. Common Diagnoses Among Male Workers in 2010}

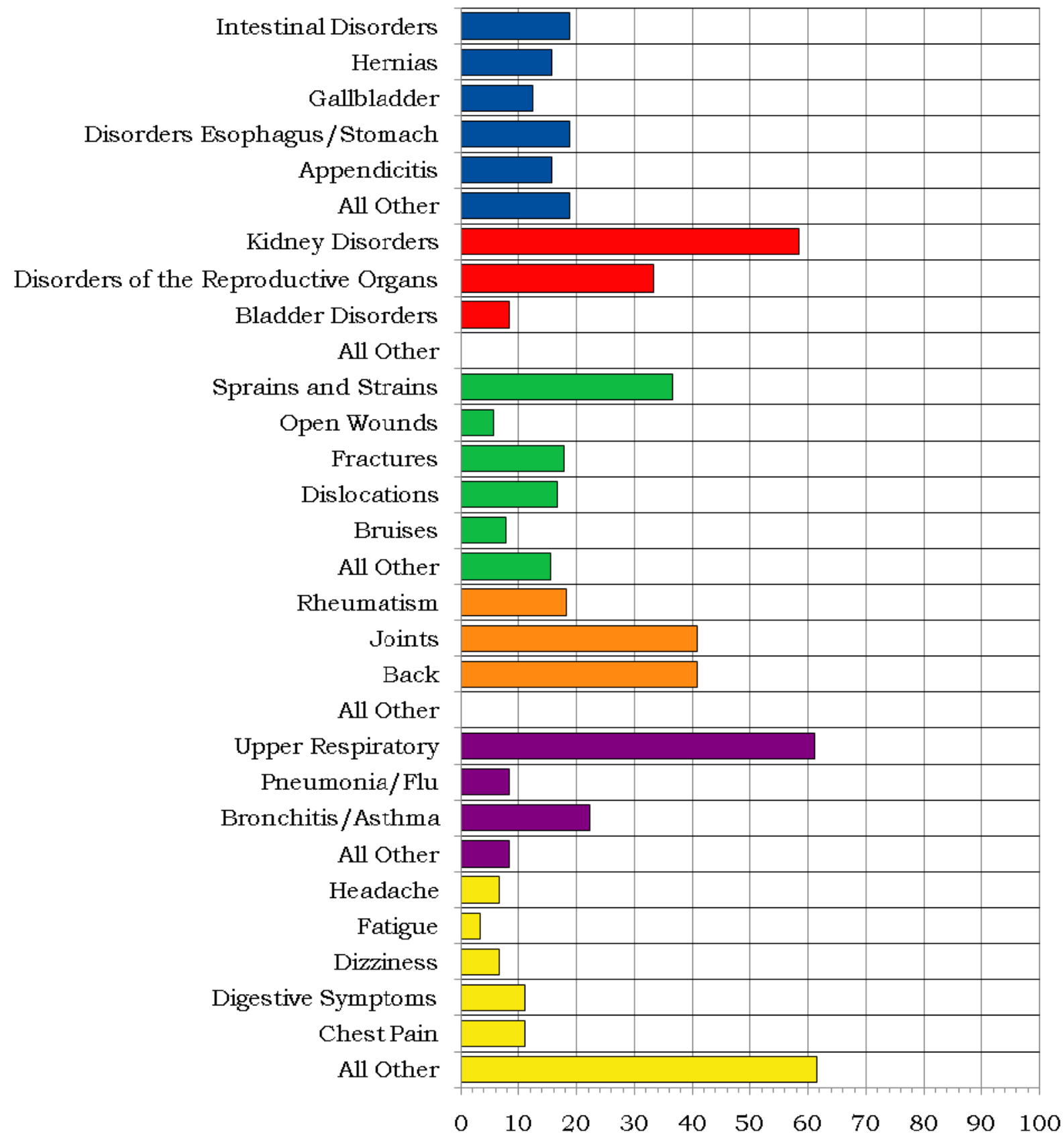

Percent Distribution of Diagnoses Within Diagnostic Category

Digestive, 32 Diagnoses

Genitourinary, 12 Diagnoses

Injury, 90 Diagnoses
Musculoskeletal, 71 Diagnoses

Respiratory, 36 Diagnoses

Unspecified Symptoms, 91 Diagnoses 


\section{Figure 10. Number of Most Frequently Reported Diagnoses by Job Category and Gender}

\begin{tabular}{|c|c|c|c|c|}
\hline Job Category & Men & & Women & \\
\hline \multirow{3}{*}{ Professional } & Unspecified Symptoms & 39 & M usculoskeletal & 21 \\
\hline & Injury & 30 & Injury & 17 \\
\hline & M usculoskeletal & 25 & Unspecified Symptoms & 15 \\
\hline \multirow{3}{*}{ Administrative Support } & Unspecified Symptoms & 3 & Unspecified Symptoms & 12 \\
\hline & Respiratory & 1 & Digestive & 11 \\
\hline & Digestive & 1 & M usculoskeletal & 10 \\
\hline \multirow{3}{*}{ Technical Support } & Injury & 13 & Genitourinary & 7 \\
\hline & Digestive & 10 & M usculoskeletal & 6 \\
\hline & Heart/Circulatory & 6 & Injury & 5 \\
\hline \multirow{5}{*}{ Service } & Digestive & 4 & Unspecified Symptoms & 5 \\
\hline & Injury & 3 & M usculoskeletal & 4 \\
\hline & Unspecified Symptoms & 2 & Respiratory & 4 \\
\hline & Respiratory & 2 & Genitourinary & 4 \\
\hline & Heart/Circulatory & 2 & & \\
\hline \multirow{7}{*}{ Security and Fire } & Injury & 21 & Unspecified Symptoms & 4 \\
\hline & Unspecified Symptoms & 20 & Respiratory & 3 \\
\hline & M usculoskeletal & 17 & M usculoskeletal & 1 \\
\hline & & & Injury & 1 \\
\hline & & & Psychological & 1 \\
\hline & & & Infections/Parasites & 1 \\
\hline & & & Benign Growths & 1 \\
\hline \multirow{3}{*}{ Crafts } & Unspecified Symptoms & 25 & Respiratory & 7 \\
\hline & M usculoskeletal & 25 & Nervous System & 4 \\
\hline & Injury & 24 & Injury & 3 \\
\hline Line Operators & & $\mathbf{0}$ & & 0 \\
\hline
\end{tabular}




\section{Rates of Disease Occurrence}

Figure 11. Rates for All Illnesses and Injuries Combined by Job Category, Gender, and Age

\begin{tabular}{|c|c|c|c|c|}
\hline \multirow{2}{*}{$\begin{array}{c}\text { All Illnesses \& } \\
\text { Injuries Combined }\end{array}$} & \multicolumn{4}{|c|}{ Rate per 1,000} \\
\hline & Job Category & Age & Men & Women \\
\hline & \multirow{2}{*}{ Professional } & $<50$ & 42 & 170 \\
\hline & & $50+$ & 176 & 227 \\
\hline & \multirow{2}{*}{ Administrative Support } & $<50$ & 136 & 333 \\
\hline & & $50+$ & 100 & 299 \\
\hline & \multirow{2}{*}{ Technical Support } & $<50$ & 68 & 214 \\
\hline & & $50+$ & 157 & 241 \\
\hline & \multirow{2}{*}{ Service } & $<50$ & 310 & 1,545 \\
\hline & & $50+$ & 118 & 143 \\
\hline & \multirow{2}{*}{ Security and Fire } & $<50$ & 325 & 727 \\
\hline & & $50+$ & 207 & 571 \\
\hline & \multirow{2}{*}{ Crafts } & $<50$ & 135 & 227 \\
\hline & & $50+$ & 173 & 444 \\
\hline & \multirow{2}{*}{ Line Operators } & $<50$ & 0 & 0 \\
\hline & & $50+$ & 0 & 0 \\
\hline
\end{tabular}

Figure 12. Rates for Selected Diagnostic Categories by Job Category, Gender, and Age

\begin{tabular}{|c|c|c|c|c|}
\hline \multirow{2}{*}{ Cancer } & \multicolumn{4}{|c|}{ Rate per 1,000} \\
\hline & Job Category & Age & Men & Women \\
\hline & Profescional & $<50$ & 0 & 0 \\
\hline & FIOIESSIOHAl & $50+$ & 5 & 0 \\
\hline & Adminictrotive Sunnort & $<50$ & 0 & 10 \\
\hline & Aaministrative support & $50+$ & 0 & 9 \\
\hline & Tonhnipol $\mathrm{C}$ unnomet & $<50$ & 0 & 0 \\
\hline & 1 echnical support & $50+$ & 0 & 0 \\
\hline & & $<50$ & 0 & 0 \\
\hline & Service & $50+$ & 20 & 0 \\
\hline & Security and Fire & $<50$ & 0 & 0 \\
\hline & & $50+$ & 0 & $\mathbf{0}$ \\
\hline & Crafts & $<50$ & 0 & 0 \\
\hline & & $50+$ & 6 & 56 \\
\hline & Line Onerators & $<50$ & 0 & 0 \\
\hline & & $50+$ & 0 & 0 \\
\hline
\end{tabular}


Figure 12. Rates for Selected Diagnostic Categories by Job Category, Gender, and Age (Continued)

\begin{tabular}{|c|c|c|c|c|}
\hline \multirow{2}{*}{ Heart/Circulatory } & \multicolumn{4}{|c|}{ Rate per 1,000} \\
\hline & Job Category & Age & Men & Women \\
\hline & \multirow{2}{*}{ Professional } & $<50$ & 2 & 4 \\
\hline & & $50+$ & 27 & 14 \\
\hline & \multirow{2}{*}{ Administrative Support } & $<50$ & 0 & 19 \\
\hline & & $50+$ & 0 & 47 \\
\hline & \multirow{2}{*}{ Technical Support } & $<50$ & 0 & 0 \\
\hline & & $50+$ & 25 & 0 \\
\hline & \multirow{2}{*}{ Service } & $<50$ & 0 & 0 \\
\hline & & $50+$ & 39 & 0 \\
\hline & \multirow{2}{*}{ Security and Fire } & $<50$ & 4 & 0 \\
\hline & & $50+$ & 0 & 0 \\
\hline & \multirow{2}{*}{ Crafts } & $<50$ & 3 & 0 \\
\hline & & $50+$ & 25 & 56 \\
\hline & \multirow{2}{*}{ Line Operators } & $<50$ & 0 & 0 \\
\hline & & $50+$ & 0 & 0 \\
\hline
\end{tabular}

\begin{tabular}{|c|c|c|c|c|}
\hline \multirow{2}{*}{ Respiratory } & \multicolumn{4}{|c|}{ Rate per 1,000} \\
\hline & Job Category & Age & Men & Women \\
\hline & \multirow{2}{*}{ Professional } & $<50$ & 0 & 14 \\
\hline & & $50+$ & 8 & 27 \\
\hline & \multirow{2}{*}{ Administrative Support } & $<50$ & 45 & 0 \\
\hline & & $50+$ & 0 & 28 \\
\hline & \multirow{2}{*}{ Technical Support } & $<50$ & 11 & 0 \\
\hline & & $50+$ & 13 & 0 \\
\hline & \multirow{2}{*}{ Service } & $<50$ & 69 & 364 \\
\hline & & $50+$ & 0 & 0 \\
\hline & \multirow{2}{*}{ Security and Fire } & $<50$ & 49 & 182 \\
\hline & & $50+$ & 34 & 143 \\
\hline & \multirow{2}{*}{ Crafts } & $<50$ & 6 & 91 \\
\hline & & $50+$ & 14 & 139 \\
\hline & \multirow{2}{*}{ Line Operators } & $<50$ & 0 & 0 \\
\hline & & $50+$ & 0 & 0 \\
\hline
\end{tabular}


Figure 12. Rates for Selected Diagnostic Categories by Job Category, Gender, and Age (Continued)

\begin{tabular}{|c|c|c|c|c|}
\hline \multirow{2}{*}{ Injury } & \multicolumn{4}{|c|}{ Rate per 1,000} \\
\hline & Job Category & Age & Men & Women \\
\hline & \multirow{2}{*}{ Professional } & $<50$ & 6 & 40 \\
\hline & & $50+$ & 33 & 21 \\
\hline & \multirow{2}{*}{ Administrative Support } & $<50$ & 0 & 29 \\
\hline & & $50+$ & 0 & 28 \\
\hline & \multirow{2}{*}{ Technical Support } & $<50$ & 32 & 54 \\
\hline & & $50+$ & 30 & 25 \\
\hline & \multirow{2}{*}{ Service } & $<50$ & 103 & 0 \\
\hline & & $50+$ & 0 & 0 \\
\hline & \multirow{2}{*}{ Security and Fire } & $<50$ & 81 & 0 \\
\hline & & $50+$ & 17 & 143 \\
\hline & \multirow{2}{*}{ Crafts } & $<50$ & 47 & 45 \\
\hline & & $50+$ & 17 & 56 \\
\hline & \multirow{2}{*}{ Line Operators } & $<50$ & 0 & 0 \\
\hline & & $50+$ & 0 & 0 \\
\hline
\end{tabular}

\section{Time Trends}

Figure 13. Age-Adjusted Rates for All Diagnoses Combined Among Women and Men from 2006 to 2010*

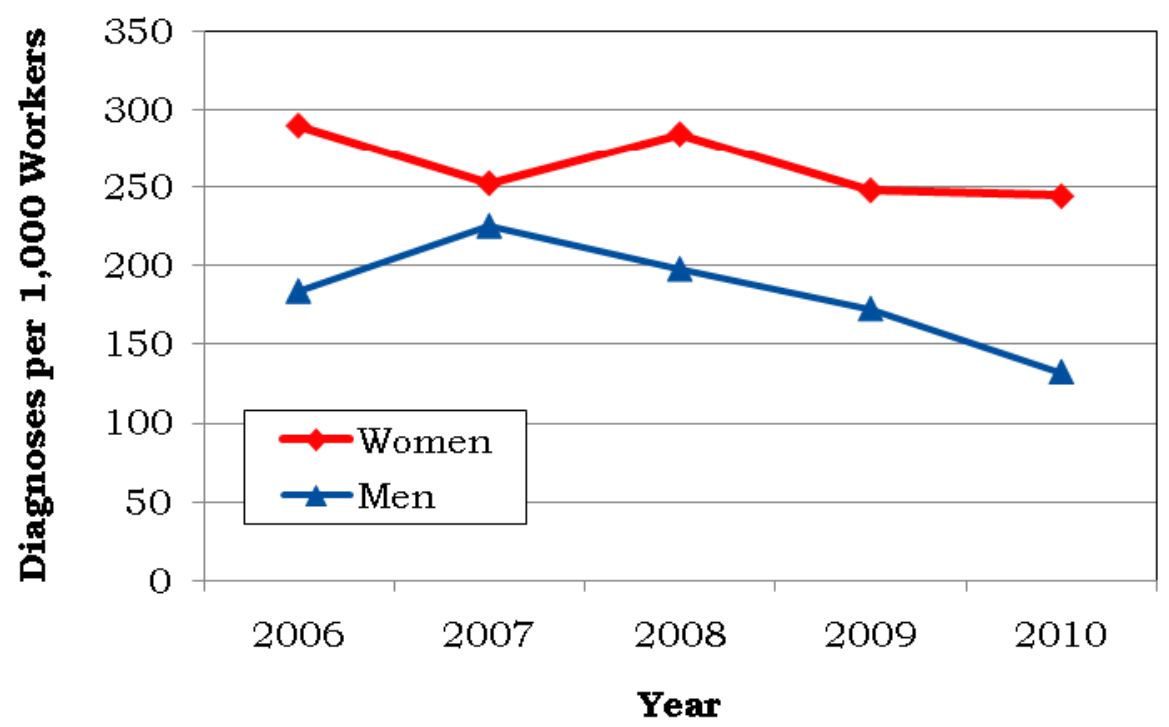

*Standardized to age distribution of 2000 U.S. population. 
Figure 14. Age-Adjusted Rates for Selected Diagnostic Categories Among Women and Men from 2006 to 2010*

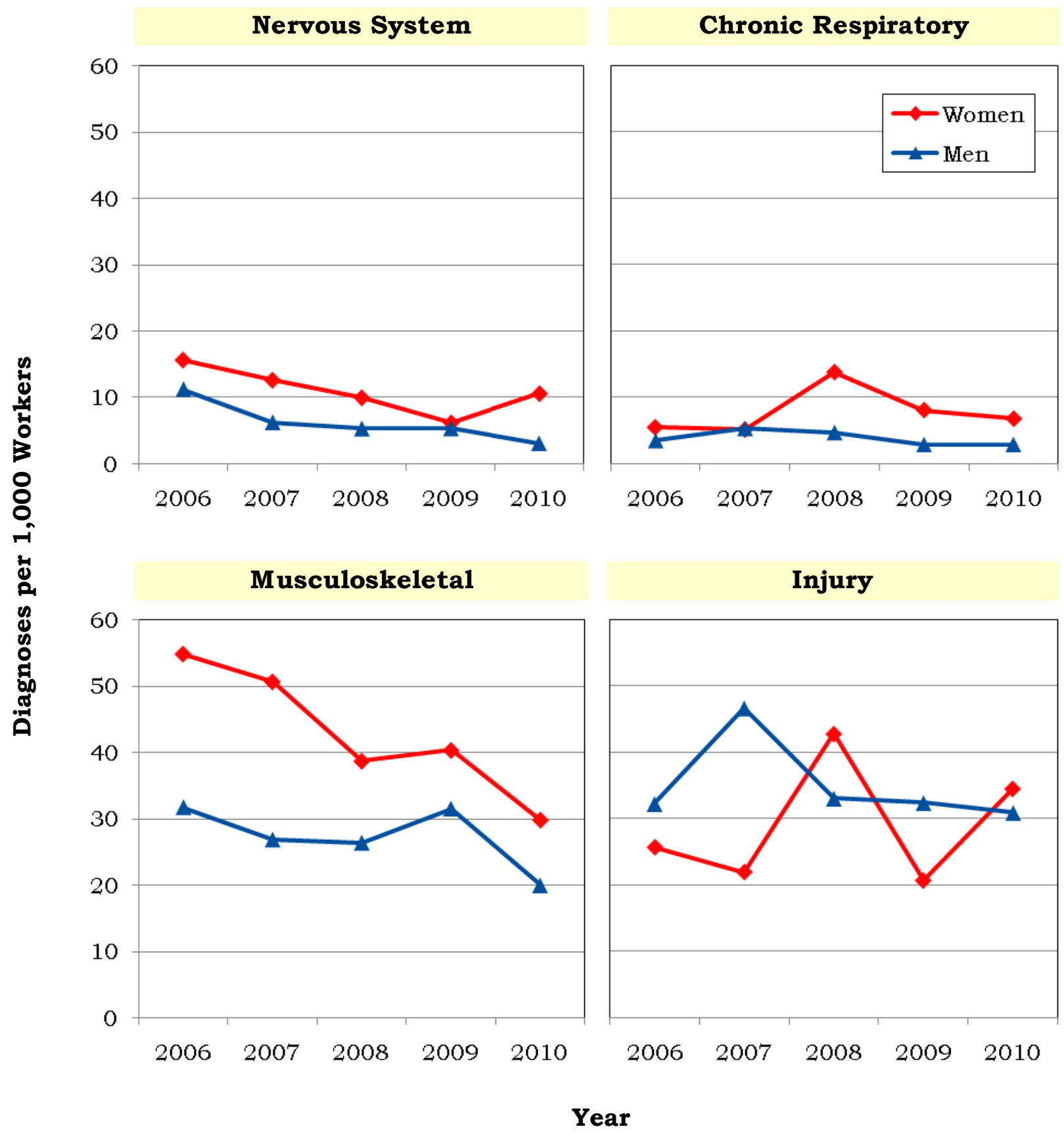

*Standardized to age distribution of 2000 U.S. population. 
Figure 15. Age-Adjusted Rates for All Diagnoses Combined Among Women and Men by Job Category from 2006 to 2010*

Professional

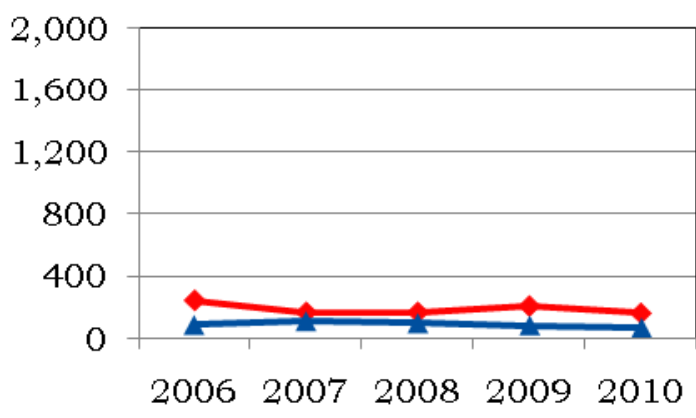

Administrative Support

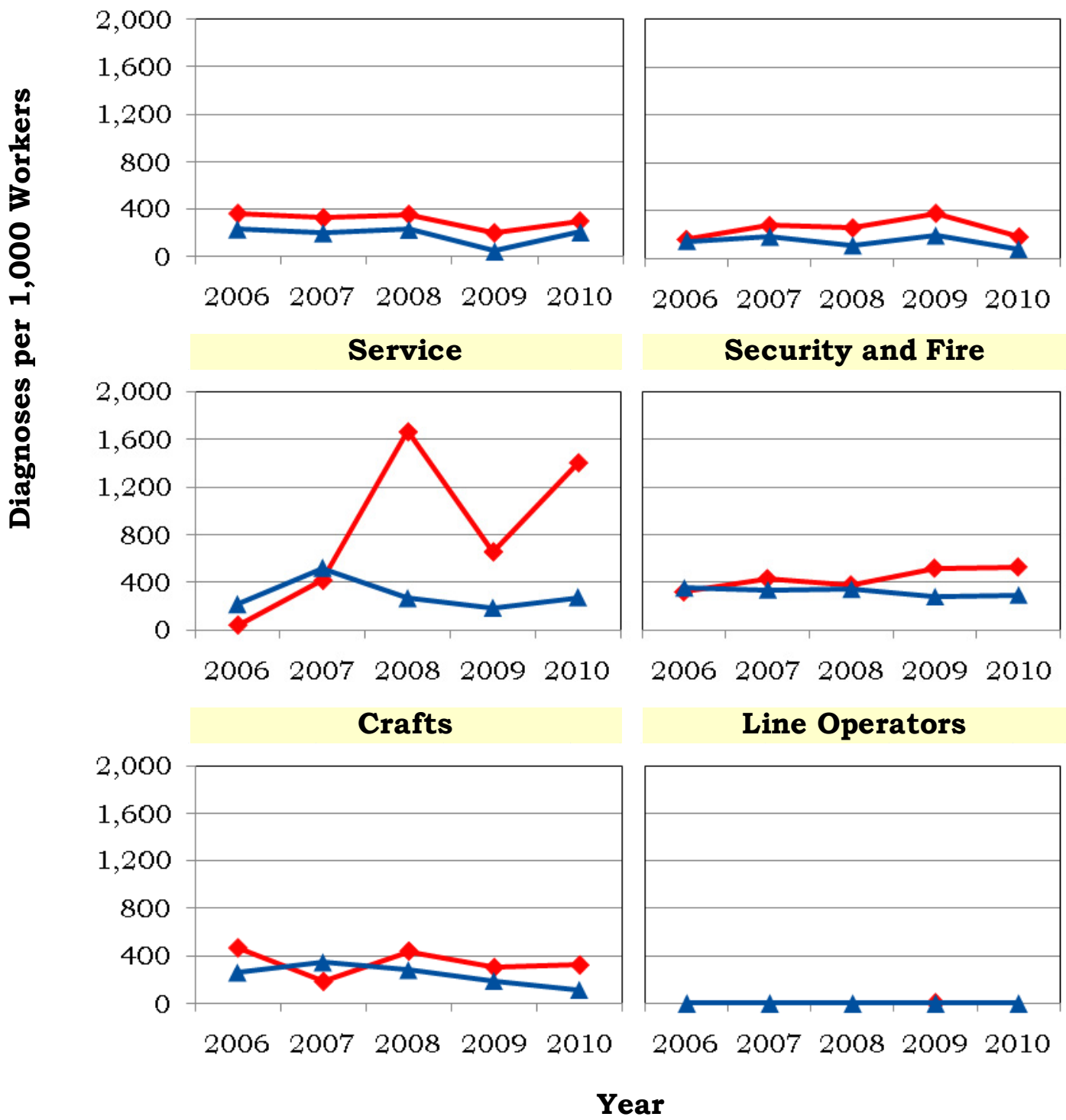

*Standardized to age distribution of 2000 U.S. population. 


\section{Sentinel Health Events for Occupations (SHEOs)}

An occupational sentinel health event (SHEO) is a disease, disability, or death that is likely to be occupationally related. Although sentinel health events may indicate an occupational exposure, many may result from nonoccupational exposures. Sentinel health events are therefore assessed in two categories:

Definite Sentinel Health Events: Diseases that are unlikely to occur in the absence of an occupational exposure (e.g., asbestosis).

Possible Sentinel Health Events: Diseases that may be occupational but can also occur in the absence of an occupational exposure (e.g., lung cancer or carpal tunnel syndrome).

Figure 16. Characteristics of SHEOs by Gender

\begin{tabular}{|l|c|c|c|c|}
\hline \multirow{2}{*}{} & \multicolumn{2}{|c|}{$\begin{array}{c}\text { Total Number of } \\
\text { SHEO Diagnoses }\end{array}$} & \multicolumn{2}{c|}{$\begin{array}{c}\text { Total Number of } \\
\text { Days Absent }\end{array}$} \\
\cline { 2 - 5 } & M en & W omen & M en & W omen \\
\hline Definite & 1 & 0 & 56 & 0 \\
\hline Possible & 2 & 6 & 13 & 114 \\
\hline Total & 3 & 6 & 69 & 114 \\
\hline
\end{tabular}

Figure 17. SHEO Diagnoses by Gender

\begin{tabular}{|l|c|c|}
\hline \multirow{2}{*}{ Diagnoses } & \multicolumn{2}{c|}{ Gender } \\
\cline { 2 - 3 } & W omen & Men \\
\hline Carpal Tunnel Syndrome & 4 & 1 \\
\hline Injuries & 0 & 1 \\
\hline Other Conditions & 2 & 1 \\
\hline
\end{tabular}


Occupational Safety and Health Administration (OSHA)-Recordable Events

Figure 18. OSHA-Recordable Events by Gender and Age

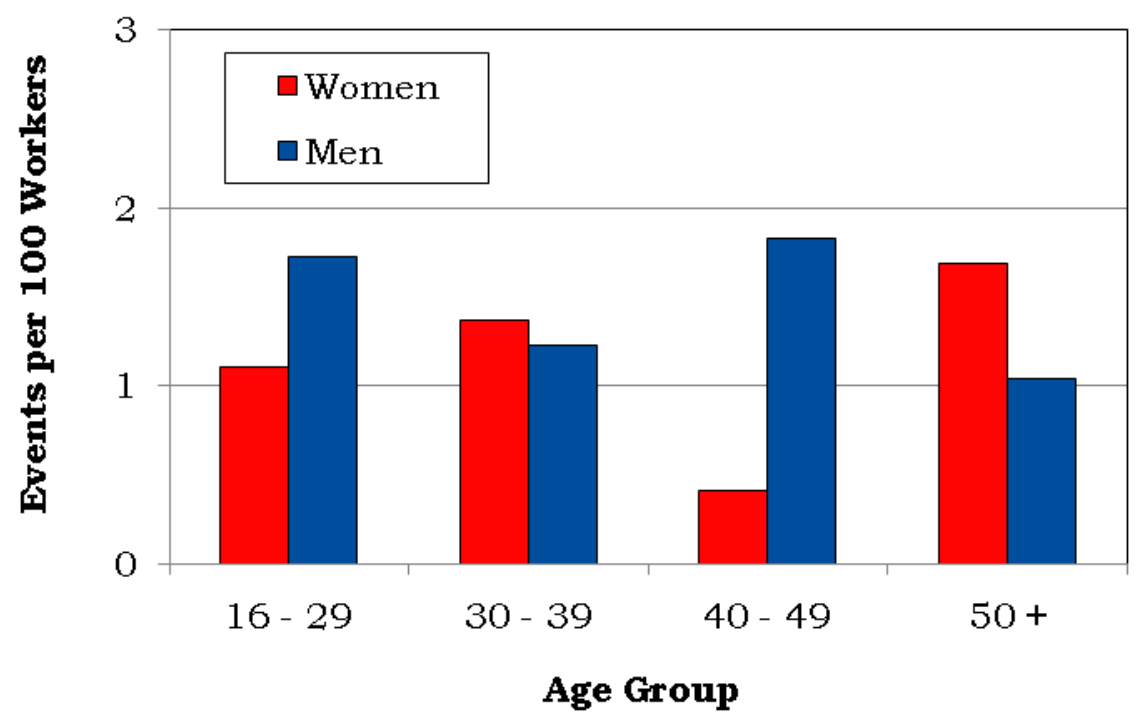

Figure 19. OSHA-Recordable Events by Job Category and Gender

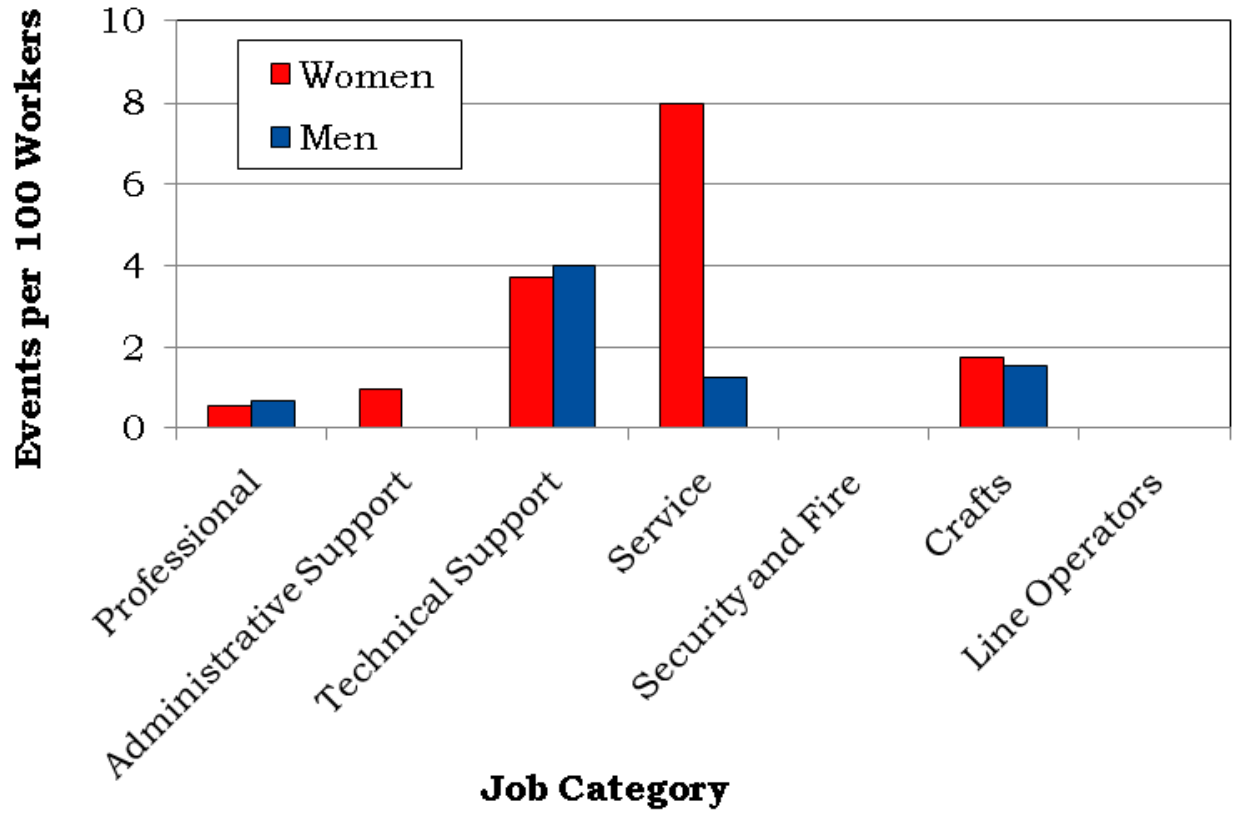




\section{Diagnostic and Accident Categories for OSHA-Recordable Events}

Figure 20. OSHA-Recordable Diagnoses by Diagnostic Category and Gender

\begin{tabular}{|l|c|c|}
\hline \multirow{2}{*}{ Diagnostic Category } & \multicolumn{2}{c|}{ Gender } \\
\cline { 2 - 3 } & Women & M en \\
\hline Musculoskeletal & $\mathbf{9}$ & $\mathbf{1 6}$ \\
\hline Nervous System & $\mathbf{0}$ & $\mathbf{1}$ \\
\hline Respiratory & $\mathbf{0}$ & $\mathbf{1}$ \\
\hline Unspecified Symptoms & $\mathbf{0}$ & $\mathbf{1}$ \\
\hline Injury & $\mathbf{1 6}$ & $\mathbf{3 9}$ \\
\hline Fractures - Upper Limb & $\mathbf{0}$ & $\mathbf{2}$ \\
\hline Fractures - Lower Limb & $\mathbf{2}$ & $\mathbf{0}$ \\
\hline Dislocations & $\mathbf{1}$ & $\mathbf{0}$ \\
\hline Back Sprains \& Strains & $\mathbf{2}$ & $\mathbf{9}$ \\
\hline Other Sprains \& Strains & $\mathbf{3}$ & $\mathbf{1 0}$ \\
\hline Intracranial Injuries & $\mathbf{0}$ & $\mathbf{1}$ \\
\hline Open Wounds - Head, Neck, Trunk & $\mathbf{0}$ & $\mathbf{4}$ \\
\hline Open Wounds - Upper Limb & $\mathbf{0}$ & $\mathbf{3}$ \\
\hline Open Wounds - Lower Limb & $\mathbf{1}$ & $\mathbf{0}$ \\
\hline Superficial Injuries & $\mathbf{1}$ & $\mathbf{2}$ \\
\hline Bruises & $\mathbf{0}$ & $\mathbf{2}$ \\
\hline Burns & $\mathbf{0}$ & $\mathbf{1}$ \\
\hline Unspecified Injuries & $\mathbf{6}$ & $\mathbf{5}$ \\
\hline
\end{tabular}


Figure 21. OSHA-Recordable Accidents by Type and Gender

\begin{tabular}{|l|c|c|}
\hline \multirow{2}{*}{\multicolumn{1}{c|}{ Accident Category }} & \multicolumn{2}{c|}{ Gender } \\
\cline { 2 - 3 } & Women & M en \\
\cline { 2 - 3 } & Number of & Number of \\
Accidents & Accidents \\
\hline Motor Vehicle Traffic & $\mathbf{2}$ & $\mathbf{3}$ \\
\hline Motor Vehicle Nontraffic & $\mathbf{0}$ & $\mathbf{1}$ \\
\hline Falls & $\mathbf{5}$ & $\mathbf{5}$ \\
\hline Fire & $\mathbf{0}$ & $\mathbf{1}$ \\
\hline Natural/Environmental Factors & $\mathbf{0}$ & $\mathbf{1}$ \\
\hline Not Self-Inflicted Injury & $\mathbf{0}$ & $\mathbf{2}$ \\
\hline Other Accidents & $\mathbf{6}$ & $\mathbf{2 3}$ \\
\hline Struck by an Object & $\mathbf{1}$ & $\mathbf{6}$ \\
\hline Caught Between Objects & $\mathbf{0}$ & $\mathbf{2}$ \\
\hline Cutting/Piercing Instrument/Object & $\mathbf{0}$ & $\mathbf{2}$ \\
\hline Overexertion/Strenuous Movements & $\mathbf{2}$ & $\mathbf{1 3}$ \\
\hline Repetitive Trauma & $\mathbf{3}$ & $\mathbf{0}$ \\
\hline Total & $\mathbf{1 3}$ & $\mathbf{3 6}$ \\
\hline
\end{tabular}




\section{Rates of OSHA-Recordable Events}

Figure 22. OSHA-Recordable Rates by Age and Job Categories Among Women, All Diagnoses Combined

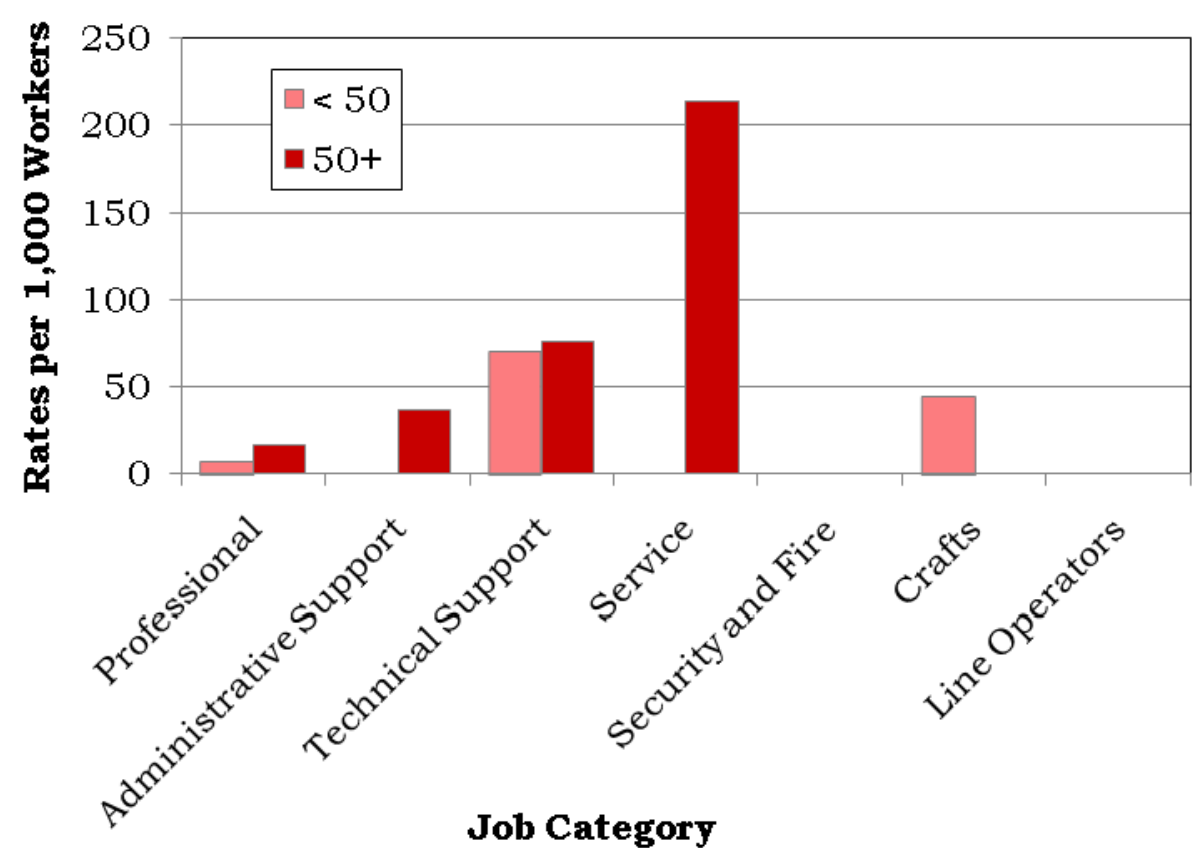

Figure 23. OSHA-Recordable Rates by Age and Job Categories Among Men, All Diagnoses Combined

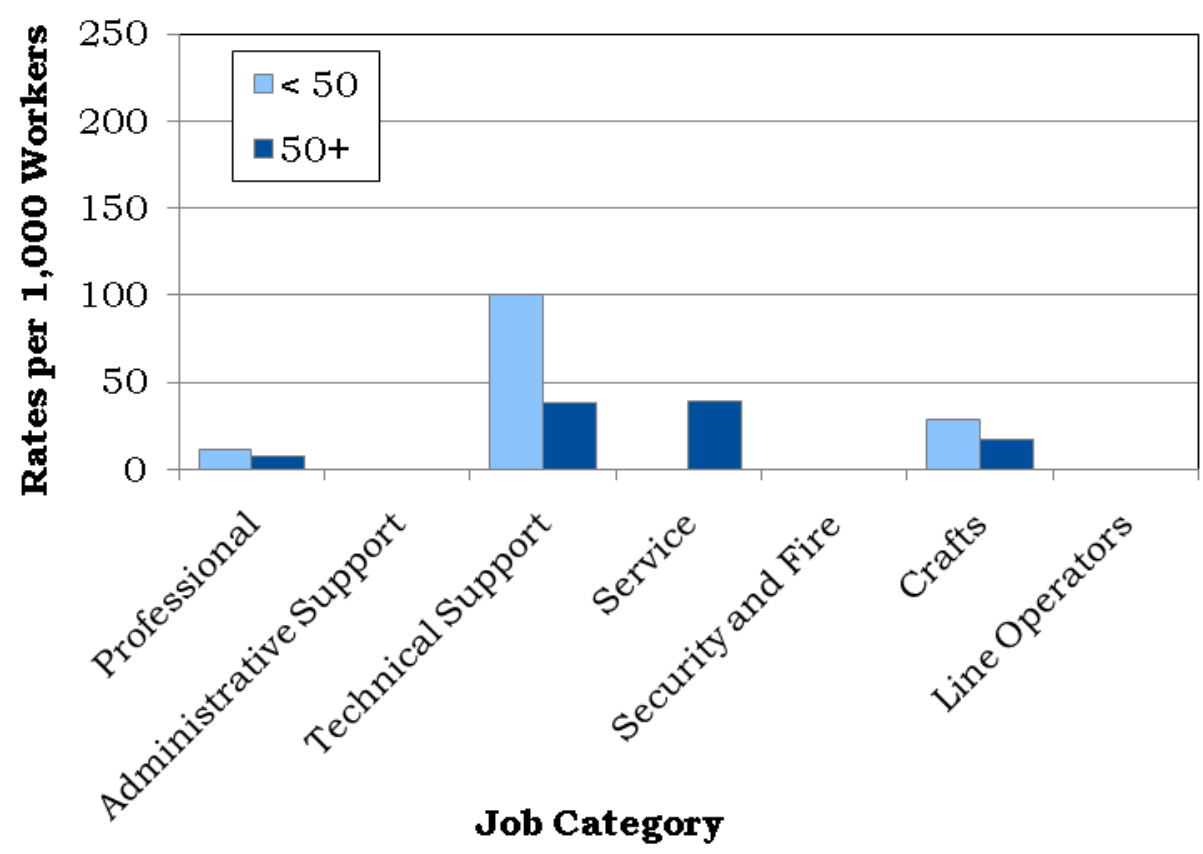




\section{Time Trends for OSHA-Recordable Events}

Figure 24. Age-Adjusted Rates for All OSHA-Recordable Diagnoses Combined Among Women and Men by Job Category from 2006 to 2010*

Professional

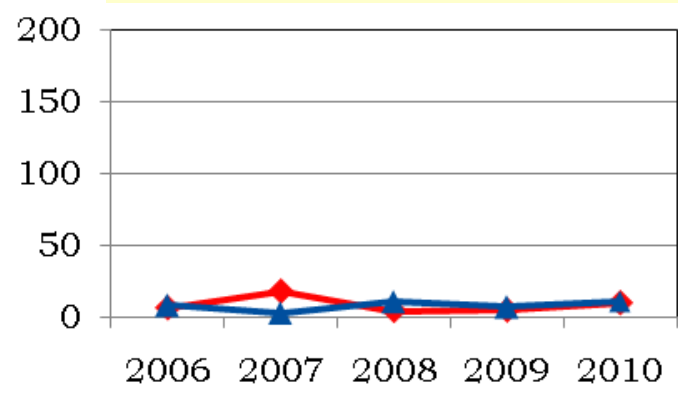

Administrative Supp ort

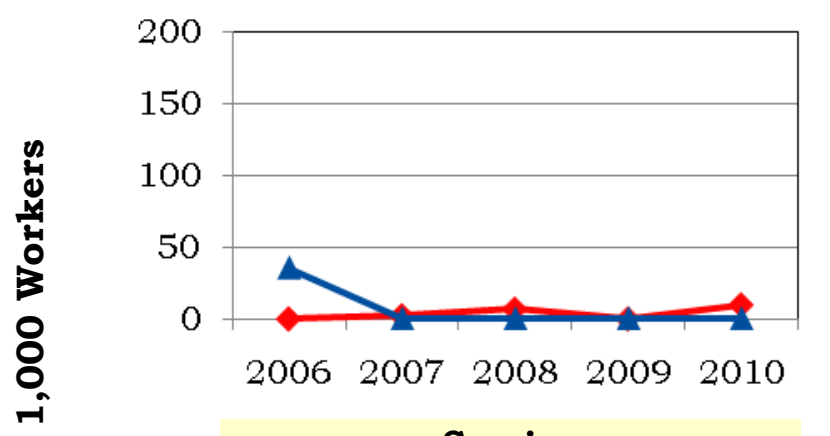

Service
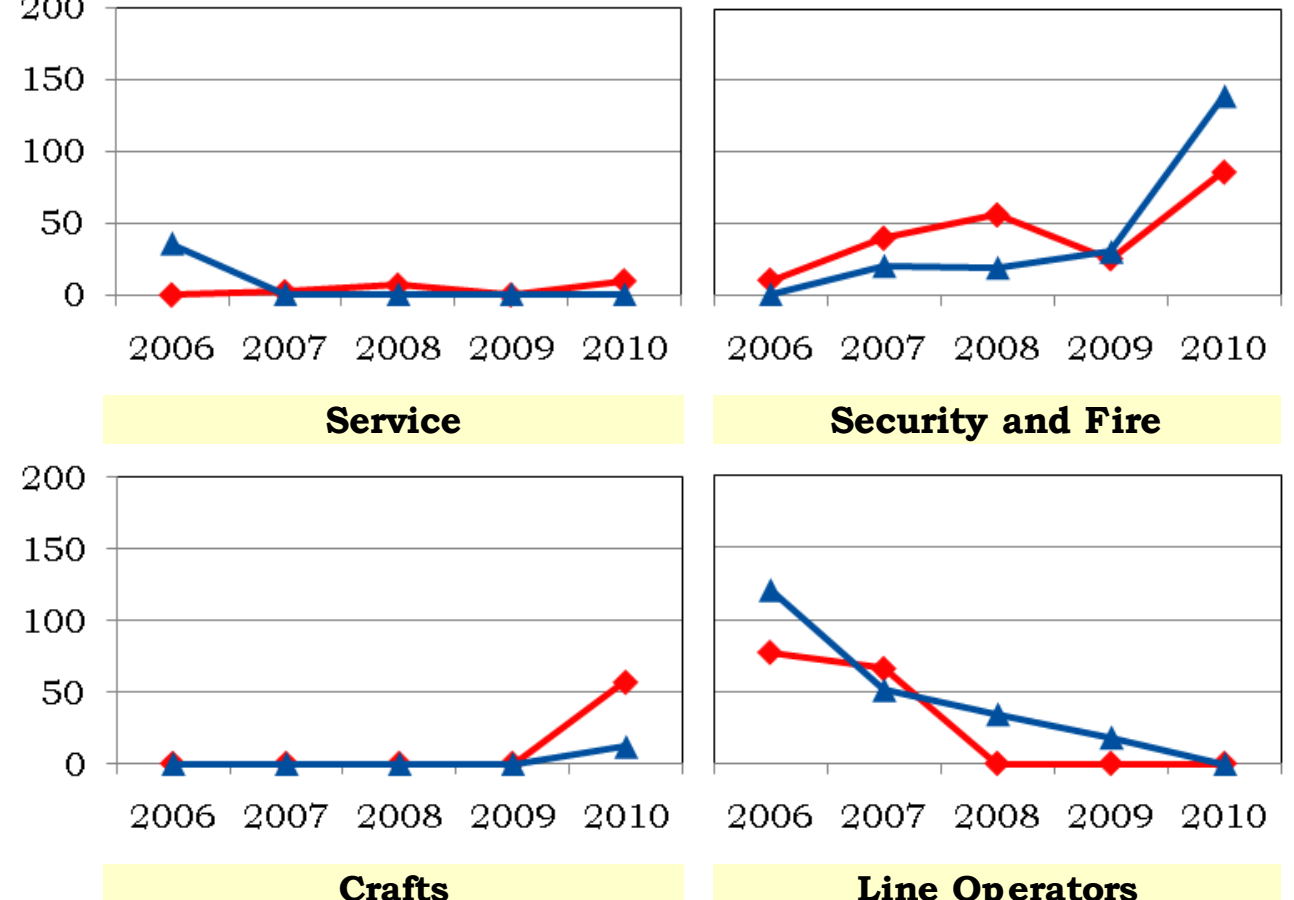

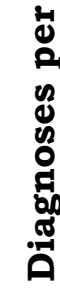

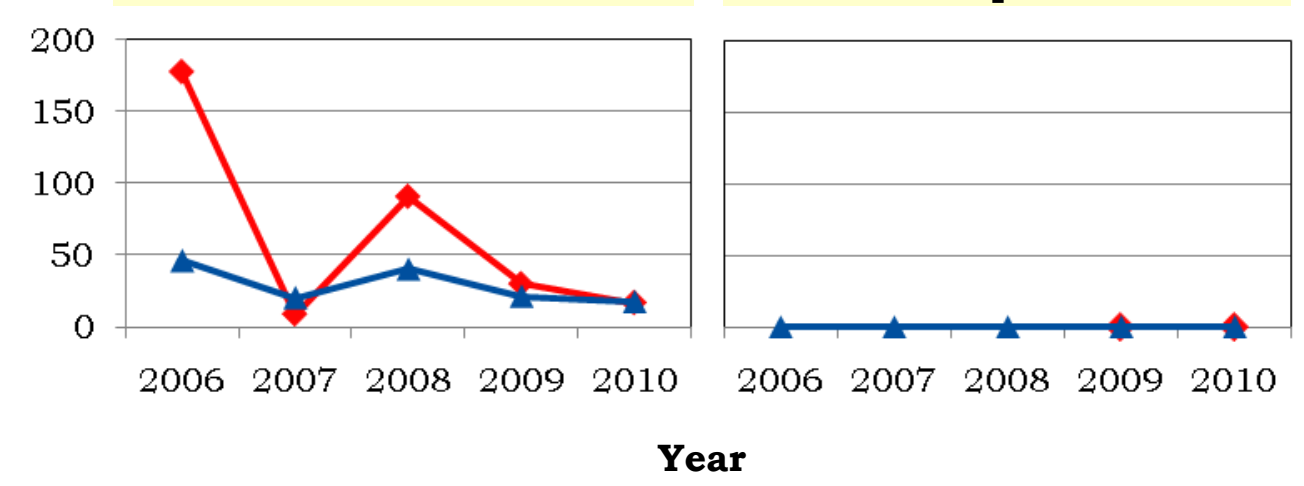

Security and Fire

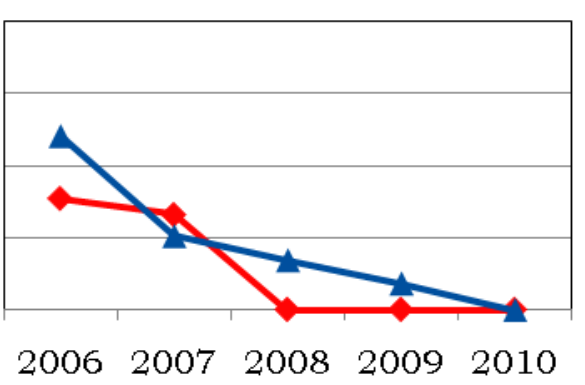

Line Operators

*Standardized to age distribution of 2000 U.S. population. 
Appendices 
Nevada National Security Site 2010

Absence Data

Appendix A. Work Force by Gender, Age, and Job Category

\begin{tabular}{|c|c|c|c|c|c|c|c|c|c|c|c|}
\hline \multirow{3}{*}{ Job Category } & \multicolumn{5}{|c|}{ Women } & \multicolumn{5}{|c|}{ Men } & \multirow[b]{3}{*}{ TOTAL } \\
\hline & \multicolumn{4}{|c|}{ Age Group } & \multirow[b]{2}{*}{ TOTAL } & \multicolumn{4}{|c|}{ Age Group } & \multirow[b]{2}{*}{ TOTAL } & \\
\hline & $16-29$ & 30 - 39 & $40-49$ & $50+$ & & $16-29$ & $30-39$ & $40-49$ & $\mathbf{5 0 +}$ & & \\
\hline Professional & 43 & 90 & 144 & 291 & 568 & 89 & 187 & 251 & 824 & 1,351 & 1,919 \\
\hline Administrative Support & 31 & 27 & 47 & 107 & 212 & 6 & 4 & 12 & 20 & 42 & 254 \\
\hline Technical Support & 9 & 19 & 28 & 79 & 135 & 19 & 66 & 105 & 236 & 426 & 561 \\
\hline Service & 1 & 2 & 8 & 14 & 25 & 4 & 4 & 21 & 51 & 80 & 105 \\
\hline Security and Fire & 5 & 2 & 4 & 7 & 18 & 65 & 106 & 75 & 58 & 304 & 322 \\
\hline Crafts & 2 & 6 & 14 & 36 & 58 & 50 & 120 & 192 & 353 & 715 & 773 \\
\hline Line Operators & 0 & 0 & 0 & 0 & 0 & 0 & 0 & 0 & 1 & 1 & 1 \\
\hline TOTAL & 91 & 146 & 245 & 534 & 1,016 & 233 & 487 & 656 & 1,543 & 2,919 & 3,935 \\
\hline
\end{tabular}

Appendix B. Age Distribution of the Work Force by Gender

\begin{tabular}{|l|r|r|r|r|r|r|r|r|}
\hline \multirow{3}{*}{ Year } & \multicolumn{4}{|c|}{ Women } & \multicolumn{4}{c|}{ Men } \\
\cline { 2 - 9 } & \multicolumn{2}{|c|}{ Percent Distribution by Age Group } & \multicolumn{1}{|c|}{ Percent Distribution by Age Group } \\
\cline { 2 - 9 } & $\mathbf{1 6}-\mathbf{2 9}$ & $\mathbf{3 0}-\mathbf{3 9}$ & $\mathbf{4 0}-\mathbf{4 9}$ & $\mathbf{5 0}+$ & $\mathbf{1 6}-\mathbf{2 9}$ & $\mathbf{3 0}-\mathbf{3 9}$ & $\mathbf{4 0}-\mathbf{4 9}$ & $\mathbf{5 0}+$ \\
\hline $\mathbf{2 0 0 2}$ & 12.28 & 21.63 & 31.98 & 34.11 & 8.38 & 15.54 & 29.99 & 46.09 \\
\hline $\mathbf{2 0 0 3}$ & 10.17 & 20.71 & 33.53 & 35.59 & 8.64 & 15.36 & 29.47 & 46.53 \\
\hline $\mathbf{2 0 0 4}$ & 11.39 & 19.32 & 31.41 & 37.88 & 10.98 & 14.32 & 28.90 & 45.80 \\
\hline $\mathbf{2 0 0 5}$ & 11.03 & 17.20 & 33.09 & 38.68 & 11.30 & 14.06 & 29.01 & 45.62 \\
\hline $\mathbf{2 0 0 6}$ & 9.77 & 16.16 & 32.15 & 41.92 & 12.99 & 13.76 & 27.70 & 45.55 \\
\hline $\mathbf{2 0 0 7}$ & 10.55 & 13.92 & 30.24 & 45.28 & 12.51 & 14.13 & 25.23 & 48.14 \\
\hline $\mathbf{2 0 0 8}$ & 10.38 & 14.01 & 27.93 & 47.68 & 10.99 & 14.99 & 23.54 & 50.47 \\
\hline $\mathbf{2 0 0 9}$ & 8.33 & 14.01 & 24.98 & 52.68 & 8.88 & 15.41 & 22.19 & 53.52 \\
\hline $\mathbf{2 0 1 0}$ & 8.96 & 14.37 & 24.11 & 52.56 & 7.98 & 16.68 & 22.47 & 52.86 \\
\hline
\end{tabular}


Nevada National Security Site 2010

Absence Data

Appendix C. Total Number of Workers Who Reported at Least One Absence by Gender, Age, and Job Category*

\begin{tabular}{|c|c|c|c|c|c|c|c|c|c|c|c|}
\hline \multirow{3}{*}{ Job Category } & \multicolumn{5}{|c|}{ Women } & \multicolumn{5}{|c|}{ Men } & \multirow[b]{3}{*}{ TOTAL } \\
\hline & \multicolumn{4}{|c|}{ Age Group } & \multirow[b]{2}{*}{ TOTAL } & \multicolumn{4}{|c|}{ Age Group } & \multirow[b]{2}{*}{ TOTAL } & \\
\hline & $16-29$ & 30 - 39 & $40-49$ & $50+$ & & $16-29$ & 30 - 39 & $40-49$ & $50+$ & & \\
\hline Professional & 0 & 11 & 11 & 30 & 52 & 0 & 3 & 10 & 64 & 77 & 129 \\
\hline Administrative Support & 3 & 4 & 7 & 16 & 30 & 0 & 1 & 0 & 1 & 2 & 32 \\
\hline Technical Support & 0 & 1 & 5 & 6 & 12 & 0 & 2 & 5 & 15 & 22 & 34 \\
\hline Service & 0 & 1 & 2 & 1 & 4 & 0 & 1 & 2 & 4 & 7 & 11 \\
\hline Security and Fire & 2 & 0 & 0 & 2 & 4 & 11 & 12 & 12 & 6 & 41 & 45 \\
\hline Crafts & 1 & 0 & 1 & 6 & 8 & 0 & 4 & 13 & 27 & 44 & 52 \\
\hline TOTAL & 6 & 17 & 26 & 61 & 110 & 11 & 23 & 42 & 117 & 193 & 303 \\
\hline
\end{tabular}

*Only those job categories and gender/age combinations with at least one absence appear in this table.

Appendix D. Total Number of Absences by Gender, Age, and Job Category*

\begin{tabular}{|c|c|c|c|c|c|c|c|c|c|c|c|}
\hline \multirow{3}{*}{ Job Category } & \multicolumn{5}{|c|}{ Women } & \multicolumn{5}{|c|}{ Men } & \multirow[b]{3}{*}{ TOTAL } \\
\hline & \multicolumn{4}{|c|}{ Age Group } & \multirow[b]{2}{*}{ TOTAL } & \multicolumn{4}{|c|}{ Age Group } & \multirow[b]{2}{*}{ TOTAL } & \\
\hline & $16-29$ & 30 - 39 & $40-49$ & $50+$ & & $16-29$ & $30-39$ & $40-49$ & $50+$ & & \\
\hline Professional & 0 & 14 & 11 & 38 & 63 & 0 & 3 & 12 & 74 & 89 & 152 \\
\hline Administrative Support & 3 & 4 & 11 & 18 & 36 & 0 & 1 & 0 & 1 & 2 & 38 \\
\hline Technical Support & 0 & 1 & 6 & 6 & 13 & 0 & 2 & 5 & 18 & 25 & 38 \\
\hline Service & 0 & 2 & 3 & 1 & 6 & 0 & 1 & 2 & 4 & 7 & 13 \\
\hline Security and Fire & 2 & 0 & 0 & 3 & 5 & 13 & 14 & 14 & 6 & 47 & 52 \\
\hline Crafts & 1 & 0 & 1 & 10 & 12 & 0 & 4 & 19 & 30 & 53 & 65 \\
\hline TOTAL & 6 & 21 & 32 & 76 & 135 & 13 & 25 & 52 & 133 & 223 & 358 \\
\hline
\end{tabular}

*Only those job categories and gender/age combinations with at least one absence appear in this table. 
Nevada National Security Site 2010

Absence Data

Appendix E. Distribution of the Number of Calendar Days Missed per Absence by Gender and Age*

\begin{tabular}{|c|c|c|c|c|c|c|c|c|c|c|c|}
\hline \multirow{3}{*}{$\begin{array}{c}\text { Number of } \\
\text { Calendar Days }\end{array}$} & \multicolumn{5}{|c|}{ Women } & \multicolumn{5}{|c|}{ Men } & \multirow[b]{3}{*}{ TOTAL } \\
\hline & \multicolumn{4}{|c|}{ Age Group } & \multirow[b]{2}{*}{ TOTAL } & \multicolumn{4}{|c|}{ Age Group } & \multirow[b]{2}{*}{ TOTAL } & \\
\hline & $16-29$ & $30-39$ & $40-49$ & $50+$ & & $16-29$ & $30-39$ & $40-49$ & $50+$ & & \\
\hline$<15$ & 5 & 16 & 17 & 38 & 76 & 10 & 16 & 36 & 85 & 147 & 223 \\
\hline $15-28$ & 1 & 4 & 9 & 18 & 32 & 2 & 5 & 5 & 24 & 36 & 68 \\
\hline $29-42$ & 0 & 0 & 2 & 6 & 8 & 0 & 1 & 1 & 4 & 6 & 14 \\
\hline $43-56$ & 0 & 0 & 2 & 3 & 5 & 1 & 0 & 4 & 7 & 12 & 17 \\
\hline $57-91$ & 0 & 1 & 2 & 10 & 13 & 0 & 2 & 2 & 6 & 10 & 23 \\
\hline $92-182$ & 0 & 0 & 0 & 0 & 0 & 0 & 1 & 2 & 7 & 10 & 10 \\
\hline $183+$ & 0 & 0 & 0 & 1 & 1 & 0 & 0 & 2 & 0 & 2 & 3 \\
\hline TOTAL & 6 & 21 & 32 & 76 & 135 & 13 & 25 & 52 & 133 & 223 & 358 \\
\hline
\end{tabular}

*Only those gender/age combinations with at least one absence appear in this table. 
Nevada National Security Site 2010

Absence Data

Appendix F. Distribution of the Number of Calendar Days Missed per Absence by Gender and Job Category*

Women

\begin{tabular}{|c|c|c|c|c|c|c|c|}
\hline \multirow{2}{*}{$\begin{array}{c}\text { Number of } \\
\text { Calendar Days }\end{array}$} & \multicolumn{6}{|c|}{ Job Category } & \multirow[b]{2}{*}{ TOTAL } \\
\hline & Professional & $\begin{array}{c}\text { Administrative } \\
\text { Support }\end{array}$ & Technical Support & Service & Security and Fire & Crafts & \\
\hline$<15$ & 39 & 16 & 5 & 5 & 3 & 8 & 76 \\
\hline $15-28$ & 15 & 12 & 3 & 1 & 0 & 1 & 32 \\
\hline $29-42$ & 2 & 3 & 2 & 0 & 1 & 0 & 8 \\
\hline $43-56$ & 3 & 0 & 2 & 0 & 0 & 0 & 5 \\
\hline $57-91$ & 4 & 5 & 1 & 0 & 1 & 2 & 13 \\
\hline $92-182$ & 0 & 0 & 0 & 0 & 0 & 0 & 0 \\
\hline $183+$ & 0 & 0 & 0 & 0 & 0 & 1 & 1 \\
\hline TOTAL & 63 & 36 & 13 & 6 & 5 & 12 & 135 \\
\hline
\end{tabular}

Men

\begin{tabular}{|c|c|c|c|c|c|c|c|}
\hline \multirow{2}{*}{$\begin{array}{c}\text { Number of } \\
\text { Calendar Days }\end{array}$} & \multicolumn{6}{|c|}{ Job Category } & \multirow[b]{2}{*}{ TOTAL } \\
\hline & Professional & $\begin{array}{c}\text { Administrative } \\
\text { Support }\end{array}$ & Technical Support & Service & Security and Fire & Crafts & \\
\hline$<15$ & 61 & 1 & 14 & 5 & 35 & 31 & 147 \\
\hline $15-28$ & 16 & 1 & 5 & 1 & 5 & 8 & 36 \\
\hline $29-42$ & 3 & 0 & 0 & 0 & 2 & 1 & 6 \\
\hline $43-56$ & 5 & 0 & 1 & 0 & 1 & 5 & 12 \\
\hline $57-91$ & 3 & 0 & 2 & 1 & 2 & 2 & 10 \\
\hline $92-182$ & 1 & 0 & 3 & 0 & 1 & 5 & 10 \\
\hline $183+$ & 0 & 0 & 0 & 0 & 1 & 1 & 2 \\
\hline TOTAL & 89 & 2 & 25 & 7 & 47 & 53 & 223 \\
\hline
\end{tabular}

*Only those gender/job category combinations with at least one absence appear in this table. 
Nevada National Security Site 2010

Absence Data

Appendix G. Number of Diagnoses in Each Diagnostic Category by Gender and Age*

\begin{tabular}{|c|c|c|c|c|c|c|}
\hline & & \multicolumn{5}{|c|}{ Women } \\
\hline & & \multicolumn{4}{|c|}{ Age Group } & \multirow[b]{2}{*}{ TOTAL } \\
\hline & & $16-29$ & $30-39$ & $40-49$ & $50+$ & \\
\hline Diagnostic Category & ICD-9-CM Code & \multirow[b]{2}{*}{1} & \multirow[b]{2}{*}{3} & \multirow[b]{2}{*}{3} & \multirow[b]{2}{*}{3} & \multirow[b]{2}{*}{10} \\
\hline INFECTIOUS \& PARASITIC DISEASES (DIS) & 001-139 & & & & & \\
\hline -Intestinal Infectious Dis & 001-009 & 0 & 1 & 1 & 0 & 2 \\
\hline -Other Bacterial Dis & 030-041 & 0 & 0 & 2 & 0 & 2 \\
\hline -Viral Dis with Exanthem & 050-059 & 0 & 0 & 0 & 0 & 0 \\
\hline -Other Viral Dis \& Chlamydiae & 070-079 & 1 & 1 & 0 & 3 & 5 \\
\hline -Mycoses & $110-118$ & 0 & 1 & 0 & 0 & 1 \\
\hline -Other Infections \& Parasitic Dis & $130-136$ & 0 & 0 & 0 & 0 & 0 \\
\hline MALIGNANT NEOPLASMS & $\begin{array}{l}140-208, \\
209.0-209.3 \\
230-234\end{array}$ & 0 & 0 & 1 & 3 & 4 \\
\hline -Lip, Oral Cavity, Pharynx & $140-149$ & 0 & 0 & 0 & 0 & 0 \\
\hline -Digestive \& Peritoneal & $150-159$ & 0 & 0 & 0 & 0 & 0 \\
\hline -Respiratory \& Intrathoracic & $160-165$ & 0 & 0 & 0 & 1 & 1 \\
\hline -Breast & 174-175 & 0 & 0 & 1 & 2 & 3 \\
\hline -Genitourinary & 179-189 & 0 & 0 & 0 & 0 & 0 \\
\hline -Other \& Unspecified Sites & $195-199,209.7$ & 0 & 0 & 0 & 0 & 0 \\
\hline -Lymphatic \& Hematopoietic & 200-208 & 0 & 0 & 0 & 0 & 0 \\
\hline BENIGN \& UNCERTAIN NEOPLASMS & $\begin{array}{l}209.4-209.6 \\
210-229,235-239\end{array}$ & 0 & 0 & 1 & 4 & 5 \\
\hline ENDOCRINE/METABOLIC/IMMUNITY & $240-279$ & 1 & 3 & 0 & 9 & 13 \\
\hline -Thyroid Gland Disorders & $240-246$ & 0 & 0 & 0 & 1 & 1 \\
\hline -Other Endocrine Gland Dis & 249-259 & 0 & 0 & 0 & 3 & 3 \\
\hline -Other Metabolic \& Immunity Disorders & $270-279$ & 1 & 3 & 0 & 5 & 9 \\
\hline BLOOD \& BLOOD-FORMING ORGANS & $280-289$ & 0 & 0 & 0 & 0 & 0 \\
\hline MENTAL DISORDERS & $290-319$ & 1 & 4 & 0 & 0 & 5 \\
\hline -Psychoses & $290-299$ & 0 & 1 & 0 & 0 & 1 \\
\hline -Non-Psychotic Disorders & $300-302,306-316$ & 1 & 2 & 0 & 0 & 3 \\
\hline -Drug Dependence & 304-305 & 0 & 1 & 0 & 0 & 1 \\
\hline NERVOUS SYSTEM (NS) \& SENSE ORGANS & $320-389$ & 0 & 1 & 4 & 9 & 14 \\
\hline -Inflammatory Dis of Central NS & $320-326$ & 0 & 0 & 0 & 0 & 0 \\
\hline -Other Disorders of Central NS & $340-349$ & 0 & 0 & 0 & 0 & 0 \\
\hline -Disorders of Peripheral NS & $350-359$ & 0 & 0 & 2 & 5 & 7 \\
\hline -Disorders of Eye & $360-379$ & 0 & 0 & 1 & 3 & 4 \\
\hline -Diseases of Ear \& Mastoid & $380-389$ & 0 & 1 & 1 & 1 & 3 \\
\hline CIRCULATORY SYSTEM & $390-459$ & 0 & 0 & 3 & 11 & 14 \\
\hline -Hypertensive Dis & $401-405$ & 0 & 0 & 2 & 3 & 5 \\
\hline -Ischemic Heart Dis & $410-414$ & 0 & 0 & 0 & 2 & 2 \\
\hline -Other Heart Dis & $420-429$ & 0 & 0 & 0 & 4 & 4 \\
\hline
\end{tabular}

(Continued)

*Only those diagnostic categories and gender/age combinations with at least one occurrence appear in this table. 
Nevada National Security Site 2010

Absence Data

Appendix G. Number of Diagnoses in Each Diagnostic Category by Gender and Age*

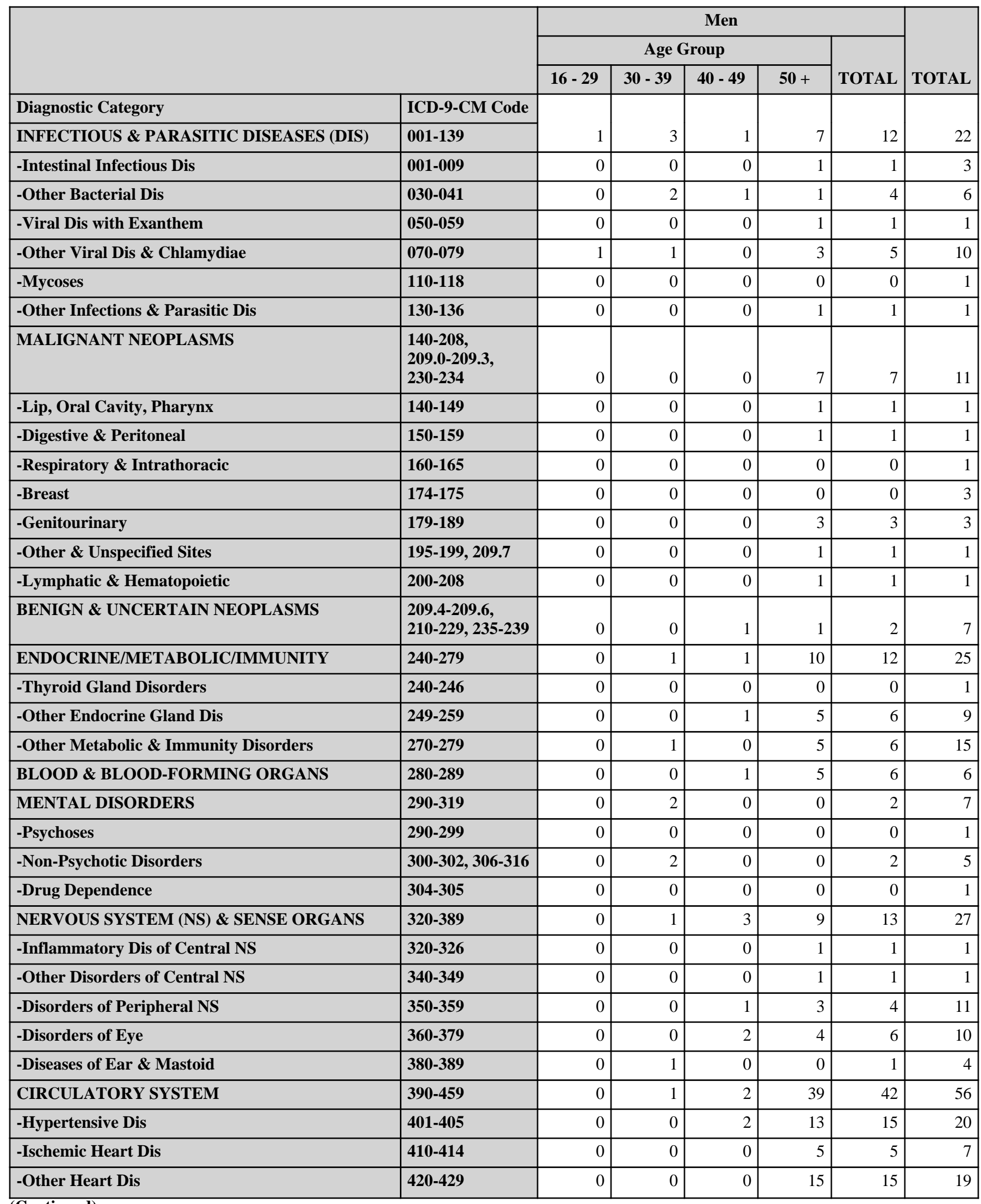

(Continued)

*Only those diagnostic categories and gender/age combinations with at least one occurrence appear in this table. 
Nevada National Security Site 2010

Absence Data

Appendix G. Number of Diagnoses in Each Diagnostic Category by Gender and Age*

\begin{tabular}{|c|c|c|c|c|c|c|}
\hline & & \multicolumn{5}{|c|}{ Women } \\
\hline & & \multicolumn{4}{|c|}{ Age Group } & \multirow[b]{2}{*}{ TOTAL } \\
\hline & & $16-29$ & $30-39$ & $40-49$ & $50+$ & \\
\hline Diagnostic Category & ICD-9-CM Code & \multirow[b]{2}{*}{0} & \multirow[b]{2}{*}{0} & \multirow[b]{2}{*}{0} & \multirow[b]{2}{*}{1} & \multirow[b]{2}{*}{1} \\
\hline -Cerebrovascular Dis & $430-438$ & & & & & \\
\hline -Dis of Arteries \& Capillaries & $440-449$ & 0 & 0 & 0 & 1 & 1 \\
\hline -Dis of Veins, Lymphatics, Other & $451-459$ & 0 & 0 & 1 & 0 & 1 \\
\hline RESPIRATORY SYSTEM & $460-519$ & 2 & 5 & 5 & 17 & 29 \\
\hline -Acute Respiratory Infections & $460-466$ & 2 & 2 & 1 & 1 & 6 \\
\hline -Other Dis Upper Respiratory Tract & $470-478$ & 0 & 0 & 3 & 8 & 11 \\
\hline -Pneumonia \& Influenza & $480-488$ & 0 & 1 & 0 & 2 & 3 \\
\hline -Chronic Obstructive Dis & $490-496$ & 0 & 2 & 1 & 5 & 8 \\
\hline -Other Respiratory Dis & $510-519$ & 0 & 0 & 0 & 1 & 1 \\
\hline DIGESTIVE SYSTEM & $520-579$ & 0 & 1 & 11 & 13 & 25 \\
\hline -Oral Cavity, Saliva Glands, Jaw & $520-529$ & 0 & 0 & 3 & 0 & 3 \\
\hline -Esophagus, Stomach, Duodenum & $530-538$ & 0 & 0 & 1 & 0 & 1 \\
\hline -Appendicitis & $540-543$ & 0 & 1 & 0 & 2 & 3 \\
\hline -Hernias & $550-553$ & 0 & 0 & 1 & 1 & 2 \\
\hline -Enteritis, Colitis & $555-558$ & 0 & 0 & 1 & 2 & 3 \\
\hline -Other Intestinal Dis & $560-569$ & 0 & 0 & 1 & 2 & 3 \\
\hline -Other Digestive Dis & $570-579$ & 0 & 0 & 4 & 6 & 10 \\
\hline GENITOURINARY SYSTEM & $580-629$ & 0 & 5 & 16 & 3 & 24 \\
\hline -Other Urinary Dis & $590-599$ & 0 & 1 & 7 & 0 & 8 \\
\hline -Male Genital Organ Dis & $600-608$ & 0 & 0 & 0 & 0 & 0 \\
\hline -Breast Disorders & $610-612$ & 0 & 0 & 0 & 0 & 0 \\
\hline -Other Female Disorders & $617-629$ & 0 & 4 & 9 & 3 & 16 \\
\hline PREGNANCY \& CHILDBIRTH & 630-679 & 0 & 1 & 0 & 0 & 1 \\
\hline -Ectopic \& Molar Pregnancy & $630-633$ & 0 & 1 & 0 & 0 & 1 \\
\hline SKIN AND SUBCUTANEOUS TISSUE & $680-709$ & 0 & 0 & 1 & 1 & 2 \\
\hline -Infections & $680-686$ & 0 & 0 & 0 & 0 & 0 \\
\hline -Other Inflammatory Conditions & 690-698 & 0 & 0 & 0 & 1 & 1 \\
\hline -Other & 700-709 & 0 & 0 & 1 & 0 & 1 \\
\hline $\begin{array}{l}\text { MUSCULOSKELETAL \& CONNECTIVE } \\
\text { TISSUE }\end{array}$ & 710-739 & 0 & 4 & 7 & 33 & 44 \\
\hline -Arthropathies & 710-719 & 0 & 2 & 1 & 14 & 17 \\
\hline -Dorsopathies & $720-724$ & 0 & 2 & 5 & 10 & 17 \\
\hline -Rheumatism, Excluding Back & 725-729 & 0 & 0 & 1 & 9 & 10 \\
\hline CONGENITAL ANOMALIES & $740-759$ & 0 & 0 & 0 & 0 & 0 \\
\hline $\begin{array}{l}\text { SYMPTOMS, SIGNS, \& ILL-DEFINED } \\
\text { CONDITIONS }\end{array}$ & 780-799 & 6 & 10 & 6 & 19 & 41 \\
\hline -Symptoms & 780-789 & 6 & 9 & 6 & 16 & 37 \\
\hline
\end{tabular}

(Continued)

*Only those diagnostic categories and gender/age combinations with at least one occurrence appear in this table. 
Nevada National Security Site 2010

Absence Data

Appendix G. Number of Diagnoses in Each Diagnostic Category by Gender and Age*

\begin{tabular}{|c|c|c|c|c|c|c|c|}
\hline & & \multicolumn{5}{|c|}{ Men } & \multirow[b]{3}{*}{ TOTAL } \\
\hline & & \multicolumn{4}{|c|}{ Age Group } & \multirow[b]{2}{*}{ TOTAL } & \\
\hline & & $16-29$ & $30-39$ & $40-49$ & $50+$ & & \\
\hline -Cerebrovascular Dis & $430-438$ & 0 & 0 & 0 & 4 & 4 & 5 \\
\hline -Dis of Arteries \& Capillaries & $440-449$ & 0 & 0 & 0 & 1 & 1 & 2 \\
\hline RESPIRATORY SYSTEM & $460-519$ & 3 & 7 & 9 & 17 & 36 & 65 \\
\hline -Acute Respiratory Infections & $460-466$ & 0 & 4 & 2 & 2 & 8 & 14 \\
\hline -Other Dis Upper Respiratory Tract & $470-478$ & 2 & 2 & 3 & 7 & 14 & 25 \\
\hline -Pneumonia \& Influenza & $480-488$ & 0 & 0 & 1 & 2 & 3 & 6 \\
\hline -Oral Cavity, Saliva Glands, Jaw & $520-529$ & 1 & 1 & 0 & 2 & 4 & 7 \\
\hline -Esophagus, Stomach, Duodenum & $530-538$ & 1 & 1 & 3 & 1 & 6 & 7 \\
\hline -Appendicitis & $540-543$ & 0 & 3 & 0 & 2 & 5 & 8 \\
\hline -Hernias & $550-553$ & 0 & 0 & 1 & 4 & 5 & 7 \\
\hline -Enteritis, Colitis & $555-558$ & 0 & 0 & 0 & 0 & 0 & 3 \\
\hline -Other Intestinal Dis & $560-569$ & 0 & 0 & 3 & 3 & 6 & 9 \\
\hline -Other Digestive Dis & $570-579$ & 0 & 1 & 1 & 4 & 6 & 16 \\
\hline GENITOURINARY SYSTEM & $580-629$ & 1 & 0 & 0 & 11 & 12 & 36 \\
\hline -Ectopic \& Molar Pregnancy & $630-633$ & 0 & 0 & 0 & 0 & 0 & 1 \\
\hline SKIN AND SUBCUTANEOUS TISSUE & $680-709$ & 1 & 0 & 3 & 5 & 9 & 11 \\
\hline -Infections & $680-686$ & 1 & 0 & 2 & 3 & 6 & 6 \\
\hline -Other Inflammatory Conditions & $690-698$ & 0 & 0 & 1 & 0 & 1 & 2 \\
\hline -Other & $700-709$ & 0 & 0 & 0 & 2 & 2 & 3 \\
\hline $\begin{array}{l}\text { MUSCULOSKELETAL \& CONNECTIVE } \\
\text { TISSUE }\end{array}$ & $710-739$ & 2 & 8 & 18 & 43 & 71 & 115 \\
\hline -Arthropathies & $710-719$ & 2 & 2 & 7 & 18 & 29 & 46 \\
\hline -Dorsopathies & $720-724$ & 0 & 3 & 8 & 18 & 29 & 46 \\
\hline -Rheumatism, Excluding Back & $725-729$ & 0 & 3 & 3 & 7 & 13 & 23 \\
\hline CONGENITAL ANOMALIES & $740-759$ & 0 & 0 & 2 & 0 & 2 & 2 \\
\hline $\begin{array}{l}\text { SYMPTOMS, SIGNS, \& ILL-DEFINED } \\
\text { CONDITIONS }\end{array}$ & $780-799$ & 5 & 16 & 18 & 52 & 91 & 132 \\
\hline -Symptoms & $780-789$ & 5 & 14 & 17 & 45 & 81 & 118 \\
\hline
\end{tabular}

(Continued)

*Only those diagnostic categories and gender/age combinations with at least one occurrence appear in this table. 
Nevada National Security Site 2010

Absence Data

Appendix G. Number of Diagnoses in Each Diagnostic Category by Gender and Age*

\begin{tabular}{|c|c|c|c|c|c|c|}
\hline & & \multicolumn{5}{|c|}{ Women } \\
\hline & & \multicolumn{4}{|c|}{ Age Group } & \multirow[b]{2}{*}{ TOTAL } \\
\hline & & $16-29$ & 30 - 39 & $40-49$ & $50+$ & \\
\hline Diagnostic Category & ICD-9-CM Code & \multirow[b]{2}{*}{0} & \multirow[b]{2}{*}{1} & \multirow[b]{2}{*}{0} & \multirow[b]{2}{*}{3} & \multirow[b]{2}{*}{4} \\
\hline -Non-Specific Abnormal Findings & $790-796$ & & & & & \\
\hline INJURY \& POISONING & $800-999$ & 2 & 10 & 6 & 14 & 32 \\
\hline -Fracture - Neck, Trunk & 805-809 & 0 & 0 & 0 & 0 & 0 \\
\hline -Fracture - Upper Limb & 810-819 & 1 & 1 & 0 & 0 & 2 \\
\hline -Fracture - Lower Limb & $820-829$ & 0 & 1 & 0 & 2 & 3 \\
\hline -Dislocation & $830-839$ & 0 & 0 & 1 & 2 & 3 \\
\hline -Sprains \& Strains - Back & 846-847 & 0 & 2 & 0 & 0 & 2 \\
\hline -Sprains \& Strains - Other & $840-845,848$ & 0 & 2 & 3 & 4 & 9 \\
\hline -Internal Injury - Thorax, Abdomen, Pelvis & $860-869$ & 0 & 0 & 0 & 0 & 0 \\
\hline -Open Wound - Head, Neck, Trunk & $870-879$ & 0 & 0 & 0 & 0 & 0 \\
\hline -Open Wound - Upper Limb & 880-887 & 0 & 0 & 0 & 0 & 0 \\
\hline -Open Wound - Lower Limb & 890-897 & 0 & 0 & 0 & 1 & 1 \\
\hline -Late Effects of Accident & 905-909 & 0 & 0 & 0 & 3 & 3 \\
\hline -Superficial Injury & $910-919$ & 1 & 0 & 0 & 0 & 1 \\
\hline -Contusion & $920-924$ & 0 & 1 & 1 & 0 & 2 \\
\hline -Complications \& Unspecified Injuries & 958-959 & 0 & 0 & 0 & 0 & 0 \\
\hline -Unspecified Effects - External Causes & $990-995$ & 0 & 0 & 0 & 0 & 0 \\
\hline -Complications of Surgical/Medical Care & 996-999 & 0 & 3 & 1 & 2 & 6 \\
\hline $\begin{array}{l}\text { HEALTH STATUS/HEALTH SERVICE } \\
\text { CONTACT }\end{array}$ & V01-V89 & 0 & 2 & 0 & 2 & 4 \\
\hline -Personal \& Family History & V10-V19 & 0 & 0 & 0 & 1 & 1 \\
\hline -Health Services Reproduction/Development & V20-V29 & 0 & 0 & 0 & 0 & 0 \\
\hline -Specific Procedures/Aftercare & V50-V59 & 0 & 2 & 0 & 1 & 3 \\
\hline -Examination \& Investigation & V70-V82 & 0 & 0 & 0 & 0 & 0 \\
\hline
\end{tabular}

*Only those diagnostic categories and gender/age combinations with at least one occurrence appear in this table. 
Nevada National Security Site 2010

Absence Data

Appendix G. Number of Diagnoses in Each Diagnostic Category by Gender and Age*

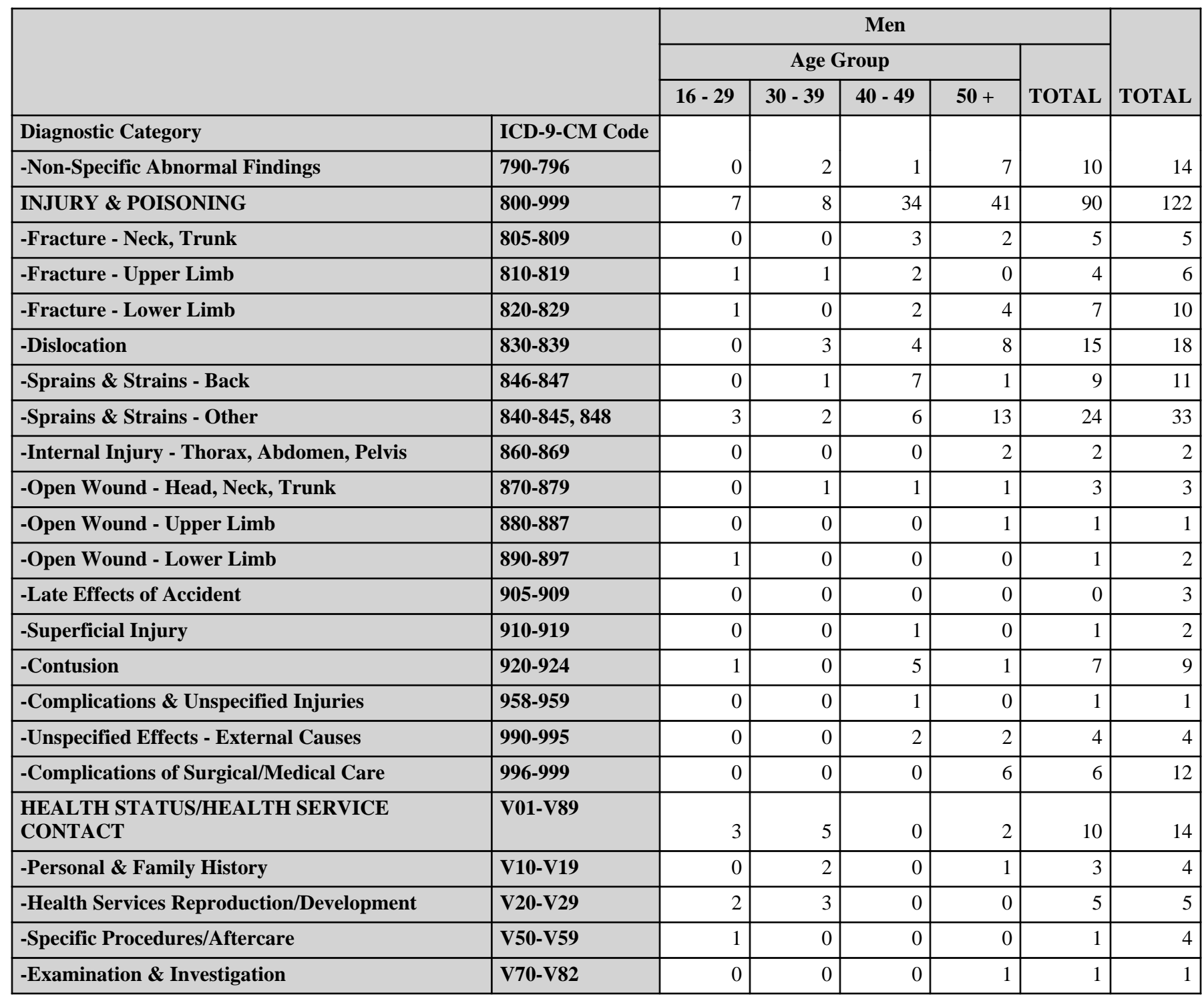

\begin{tabular}{|c|c|c|c|c|c|c|c|c|c|c|c|}
\hline & \multicolumn{5}{|c|}{ Women } & \multicolumn{5}{|c|}{ Men } & \multirow[b]{3}{*}{ TOTAL } \\
\hline & \multicolumn{4}{|c|}{ Age Group } & \multirow[b]{2}{*}{ TOTAL } & \multicolumn{4}{|c|}{ Age Group } & \multirow[b]{2}{*}{ TOTAL } & \\
\hline & $16-29$ & 30 - 39 & $40-49$ & $50+$ & & $16-29$ & 30 - 39 & $40-49$ & $50+$ & & \\
\hline Diagnostic Category & \multirow[b]{2}{*}{13} & \multirow[b]{2}{*}{49} & \multirow[b]{2}{*}{64} & \multirow[b]{2}{*}{141} & \multirow[b]{2}{*}{267} & \multirow[b]{2}{*}{25} & \multirow[b]{2}{*}{58} & \multirow[b]{2}{*}{101} & \multirow[b]{2}{*}{265} & \multirow[b]{2}{*}{449} & \multirow[b]{2}{*}{716} \\
\hline Total & & & & & & & & & & & \\
\hline
\end{tabular}

*Only those diagnostic categories and gender/age combinations with at least one occurrence appear in this table. 
Nevada National Security Site 2010

Absence Data

Appendix H. Total Number of Calendar Days Absent in Each Diagnostic Category by Gender and Age*

\begin{tabular}{|c|c|c|c|c|c|c|}
\hline & & \multicolumn{5}{|c|}{ Women } \\
\hline & & \multicolumn{4}{|c|}{ Age Group } & \multirow[b]{2}{*}{ TOTAL } \\
\hline & & $16-29$ & 30 - 39 & $40-49$ & $50+$ & \\
\hline Diagnostic Category & ICD-9-CM Code & \multirow[b]{2}{*}{2} & \multirow[b]{2}{*}{72} & \multirow[b]{2}{*}{44} & \multirow[b]{2}{*}{10} & \multirow[b]{2}{*}{128} \\
\hline INFECTIOUS \& PARASITIC DISEASES (DIS) & 001-139 & & & & & \\
\hline MALIGNANT NEOPLASMS & $\begin{array}{l}140-208, \\
209.0-209.3 \\
230-234\end{array}$ & 0 & 0 & 26 & 90 & 116 \\
\hline BENIGN \& UNCERTAIN NEOPLASMS & $\begin{array}{l}209.4-209.6 \\
210-229,235-239\end{array}$ & 0 & 0 & 27 & 154 & 181 \\
\hline ENDOCRINE/METABOLIC/IMMUNITY & $240-279$ & 19 & 38 & 0 & 90 & 147 \\
\hline BLOOD \& BLOOD-FORMING ORGANS & $280-289$ & 0 & 0 & 0 & 0 & 0 \\
\hline MENTAL DISORDERS & 290-319 & 1 & 36 & 0 & 0 & 37 \\
\hline NERVOUS SYSTEM (NS) \& SENSE ORGANS & $320-389$ & 0 & 7 & 44 & 212 & 263 \\
\hline CIRCULATORY SYSTEM & $390-459$ & 0 & 0 & 56 & 389 & 445 \\
\hline RESPIRATORY SYSTEM & $460-519$ & 2 & 13 & 30 & 141 & 186 \\
\hline DIGESTIVE SYSTEM & $520-579$ & 0 & 11 & 100 & 128 & 239 \\
\hline GENITOURINARY SYSTEM & $580-629$ & 0 & 38 & 211 & 70 & 319 \\
\hline PREGNANCY \& CHILDBIRTH & $630-679$ & 0 & 21 & 0 & 0 & 21 \\
\hline SKIN AND SUBCUTANEOUS TISSUE & 680-709 & 0 & 0 & 12 & 4 & 16 \\
\hline $\begin{array}{l}\text { MUSCULOSKELETAL \& CONNECTIVE } \\
\text { TISSUE }\end{array}$ & $710-739$ & 0 & 7 & 197 & 830 & 1,034 \\
\hline CONGENITAL ANOMALIES & $740-759$ & 0 & 0 & 0 & 0 & 0 \\
\hline $\begin{array}{l}\text { SYMPTOMS, SIGNS, \& ILL-DEFINED } \\
\text { CONDITIONS }\end{array}$ & $780-799$ & 4 & 34 & 67 & 413 & 518 \\
\hline INJURY \& POISONING & $800-999$ & 9 & 76 & 41 & 562 & 688 \\
\hline
\end{tabular}

\footnotetext{
*Absences with >1 ICD-9-CM code in the same diagnostic category were counted only once. Only those diagnostic categories and gender/age combinations with at least one occurrence appear in this table.
} 
Nevada National Security Site 2010

Absence Data

Appendix H. Total Number of Calendar Days Absent in Each Diagnostic Category by Gender and Age*

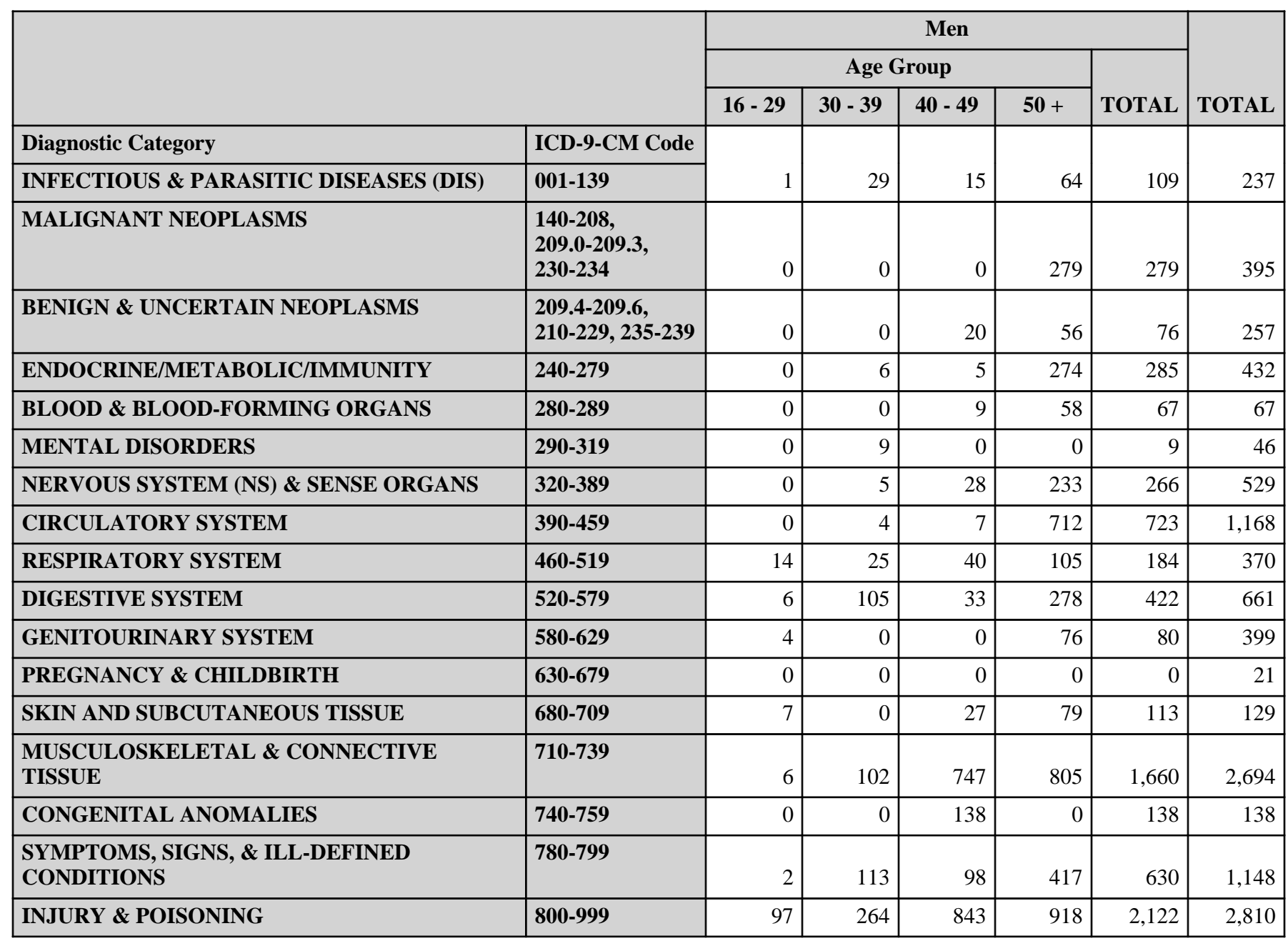

\footnotetext{
*Absences with >1 ICD-9-CM code in the same diagnostic category were counted only once. Only those diagnostic categories and gender/age combinations with at least one occurrence appear in this table.
} 
Nevada National Security Site 2010

Absence Data

Appendix I. Number of Diagnoses in Each Diagnostic Category by Gender and Job Category*

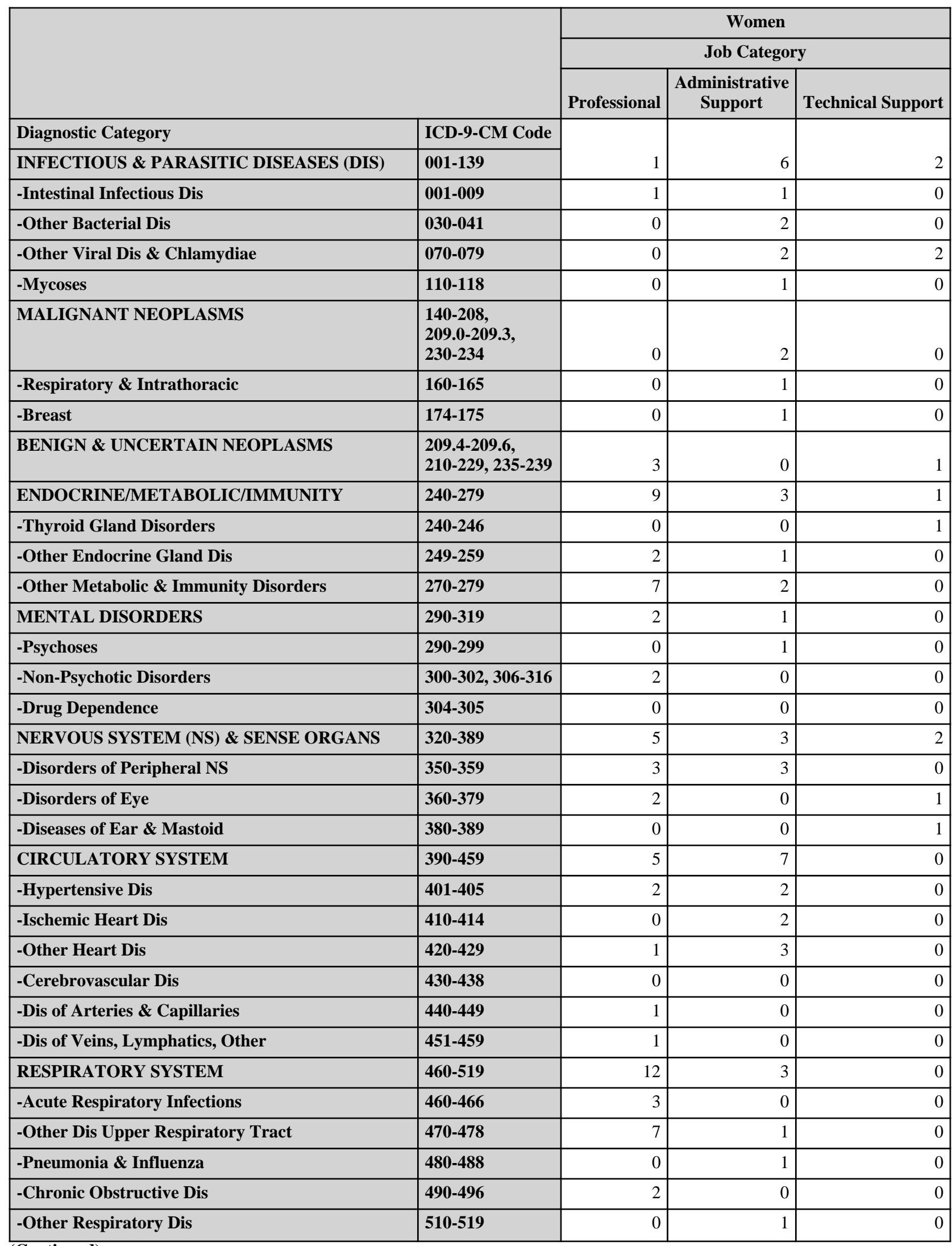

(Continued)

*Only those diagnostic categories and gender/job category combinations with at least one occurrence appear in this table. 
Nevada National Security Site 2010

Absence Data

Appendix I. Number of Diagnoses in Each Diagnostic Category by Gender and Job Category*

\begin{tabular}{|c|c|c|c|c|c|}
\hline & & \multicolumn{4}{|c|}{ Women } \\
\hline & & \multicolumn{3}{|c|}{ Job Category } & \multirow[b]{2}{*}{ TOTAL } \\
\hline & & Service & Security and Fire & Crafts & \\
\hline Diagnostic Category & ICD-9-CM Code & \multirow[b]{2}{*}{0} & \multirow[b]{2}{*}{1} & \multirow[b]{2}{*}{0} & \multirow[b]{2}{*}{10} \\
\hline INFECTIOUS \& PARASITIC DISEASES (DIS) & 001-139 & & & & \\
\hline -Intestinal Infectious Dis & 001-009 & 0 & 0 & 0 & 2 \\
\hline -Other Bacterial Dis & 030-041 & 0 & 0 & 0 & 2 \\
\hline -Other Viral Dis \& Chlamydiae & 070-079 & 0 & 1 & 0 & 5 \\
\hline -Mycoses & 110-118 & 0 & 0 & 0 & 1 \\
\hline MALIGNANT NEOPLASMS & $\begin{array}{l}140-208, \\
209.0-209.3 \\
230-234\end{array}$ & 0 & 0 & 2 & 4 \\
\hline -Respiratory \& Intrathoracic & $160-165$ & 0 & 0 & 0 & 1 \\
\hline -Breast & 174-175 & 0 & 0 & 2 & 3 \\
\hline BENIGN \& UNCERTAIN NEOPLASMS & $\begin{array}{l}209.4-209.6, \\
210-229,235-239\end{array}$ & 0 & 1 & 0 & 5 \\
\hline ENDOCRINE/METABOLIC/IMMUNITY & $240-279$ & 0 & 0 & 0 & 13 \\
\hline -Thyroid Gland Disorders & $240-246$ & 0 & 0 & 0 & 1 \\
\hline -Other Endocrine Gland Dis & 249-259 & 0 & 0 & 0 & 3 \\
\hline -Other Metabolic \& Immunity Disorders & $270-279$ & 0 & 0 & 0 & 9 \\
\hline MENTAL DISORDERS & $290-319$ & 1 & 1 & 0 & 5 \\
\hline -Psychoses & $290-299$ & 0 & 0 & 0 & 1 \\
\hline -Non-Psychotic Disorders & $300-302,306-316$ & 0 & 1 & 0 & 3 \\
\hline -Drug Dependence & $304-305$ & 1 & 0 & 0 & 1 \\
\hline NERVOUS SYSTEM (NS) \& SENSE ORGANS & $320-389$ & 0 & 0 & 4 & 14 \\
\hline -Disorders of Peripheral NS & 350-359 & 0 & 0 & 1 & 7 \\
\hline -Disorders of Eye & $360-379$ & 0 & 0 & 1 & 4 \\
\hline -Diseases of Ear \& Mastoid & 380-389 & 0 & 0 & 2 & 3 \\
\hline CIRCULATORY SYSTEM & $390-459$ & 0 & 0 & 2 & 14 \\
\hline -Hypertensive Dis & 401-405 & 0 & 0 & 1 & 5 \\
\hline -Ischemic Heart Dis & $410-414$ & 0 & 0 & 0 & 2 \\
\hline -Other Heart Dis & $420-429$ & 0 & 0 & 0 & 4 \\
\hline -Cerebrovascular Dis & $430-438$ & 0 & 0 & 1 & 1 \\
\hline -Dis of Arteries \& Capillaries & $440-449$ & 0 & 0 & 0 & 1 \\
\hline -Dis of Veins, Lymphatics, Other & 451-459 & 0 & 0 & 0 & 1 \\
\hline RESPIRATORY SYSTEM & $460-519$ & 4 & 3 & 7 & 29 \\
\hline -Acute Respiratory Infections & $460-466$ & 1 & 2 & 0 & 6 \\
\hline -Other Dis Upper Respiratory Tract & $470-478$ & 0 & 0 & 3 & 11 \\
\hline -Pneumonia \& Influenza & $480-488$ & 1 & 0 & 1 & 3 \\
\hline -Chronic Obstructive Dis & $490-496$ & 2 & 1 & 3 & 8 \\
\hline -Other Respiratory Dis & $510-519$ & 0 & 0 & 0 & 1 \\
\hline
\end{tabular}

(Continued)

*Only those diagnostic categories and gender/job category combinations with at least one occurrence appear in this table. 
Nevada National Security Site 2010

Absence Data

Appendix I. Number of Diagnoses in Each Diagnostic Category by Gender and Job Category*

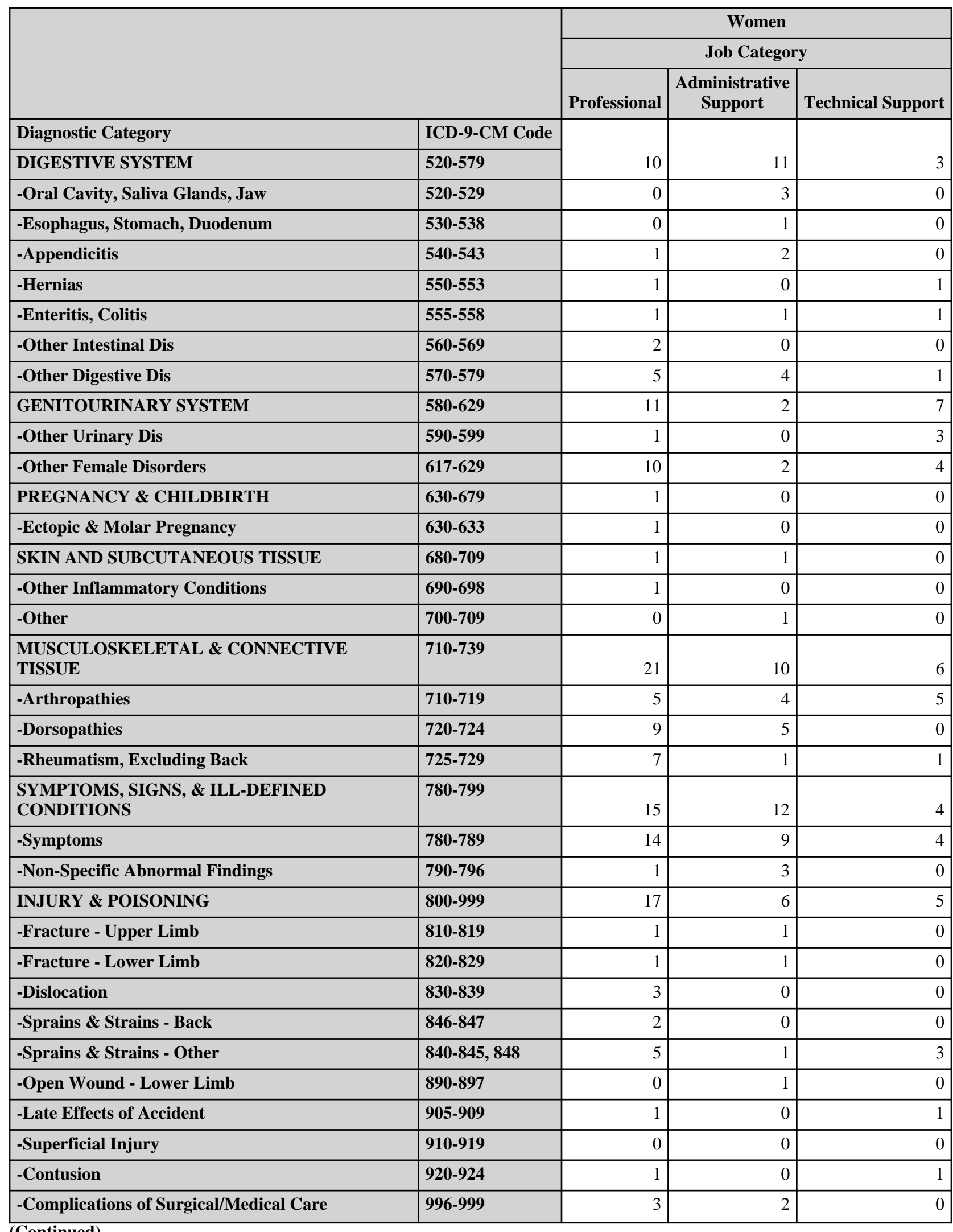

(Continued)

*Only those diagnostic categories and gender/job category combinations with at least one occurrence appear in this table. 
Nevada National Security Site 2010

Absence Data

Appendix I. Number of Diagnoses in Each Diagnostic Category by Gender and Job Category*

\begin{tabular}{|c|c|c|c|c|c|}
\hline & & \multicolumn{4}{|c|}{ Women } \\
\hline & & \multicolumn{3}{|c|}{ Job Category } & \multirow[b]{2}{*}{ TOTAL } \\
\hline & & Service & Security and Fire & Crafts & \\
\hline Diagnostic Category & ICD-9-CM Code & \multirow[b]{2}{*}{1} & \multirow[b]{2}{*}{0} & \multirow[b]{2}{*}{0} & \multirow[b]{2}{*}{25} \\
\hline DIGESTIVE SYSTEM & $520-579$ & & & & \\
\hline -Oral Cavity, Saliva Glands, Jaw & $520-529$ & 0 & 0 & 0 & 3 \\
\hline -Esophagus, Stomach, Duodenum & \begin{tabular}{|l|}
$530-538$ \\
\end{tabular} & 0 & 0 & 0 & 1 \\
\hline -Appendicitis & $540-543$ & 0 & 0 & 0 & 3 \\
\hline -Hernias & $550-553$ & 0 & 0 & 0 & 2 \\
\hline -Enteritis, Colitis & $555-558$ & 0 & 0 & 0 & 3 \\
\hline -Other Intestinal Dis & $560-569$ & 1 & 0 & 0 & 3 \\
\hline -Other Digestive Dis & $570-579$ & 0 & 0 & 0 & 10 \\
\hline GENITOURINARY SYSTEM & $580-629$ & 4 & 0 & 0 & 24 \\
\hline -Other Urinary Dis & $590-599$ & 4 & 0 & 0 & 8 \\
\hline -Other Female Disorders & 617-629 & 0 & 0 & 0 & 16 \\
\hline PREGNANCY \& CHILDBIRTH & $630-679$ & 0 & 0 & 0 & 1 \\
\hline -Ectopic \& Molar Pregnancy & $630-633$ & 0 & 0 & 0 & 1 \\
\hline SKIN AND SUBCUTANEOUS TISSUE & 680-709 & 0 & 0 & 0 & 2 \\
\hline -Other Inflammatory Conditions & 690-698 & 0 & 0 & 0 & 1 \\
\hline -Other & 700-709 & 0 & 0 & 0 & 1 \\
\hline $\begin{array}{l}\text { MUSCULOSKELETAL \& CONNECTIVE } \\
\text { TISSUE }\end{array}$ & 710-739 & 4 & 1 & 2 & 44 \\
\hline -Arthropathies & 710-719 & 3 & 0 & 0 & 17 \\
\hline -Dorsopathies & $720-724$ & 1 & 0 & 2 & 17 \\
\hline -Rheumatism, Excluding Back & 725-729 & 0 & 1 & 0 & 10 \\
\hline $\begin{array}{l}\text { SYMPTOMS, SIGNS, \& ILL-DEFINED } \\
\text { CONDITIONS }\end{array}$ & 780-799 & 5 & 4 & 1 & 41 \\
\hline- Symptoms & 780-789 & 5 & 4 & 1 & 37 \\
\hline -Non-Specific Abnormal Findings & $790-796$ & 0 & 0 & 0 & 4 \\
\hline INJURY \& POISONING & $800-999$ & 0 & 1 & 3 & 32 \\
\hline -Fracture - Upper Limb & 810-819 & 0 & 0 & 0 & 2 \\
\hline -Fracture - Lower Limb & $820-829$ & 0 & 0 & 1 & 3 \\
\hline -Dislocation & 830-839 & 0 & 0 & 0 & 3 \\
\hline -Sprains \& Strains - Back & 846-847 & 0 & 0 & 0 & 2 \\
\hline -Sprains \& Strains - Other & $840-845,848$ & 0 & 0 & 0 & 9 \\
\hline -Open Wound - Lower Limb & 890-897 & 0 & 0 & 0 & 1 \\
\hline -Late Effects of Accident & $905-909$ & 0 & 0 & 1 & 3 \\
\hline -Superficial Injury & $910-919$ & 0 & 0 & 1 & 1 \\
\hline -Contusion & $920-924$ & 0 & 0 & 0 & 2 \\
\hline -Complications of Surgical/Medical Care & 996-999 & 0 & 1 & 0 & 6 \\
\hline
\end{tabular}

(Continued)

*Only those diagnostic categories and gender/job category combinations with at least one occurrence appear in this table. 
Nevada National Security Site 2010

Absence Data

Appendix I. Number of Diagnoses in Each Diagnostic Category by Gender and Job Category*

\begin{tabular}{|c|c|c|c|c|}
\hline & & \multicolumn{3}{|c|}{ Women } \\
\hline & & \multicolumn{3}{|c|}{ Job Category } \\
\hline & & Professional & $\begin{array}{l}\text { Administrative } \\
\text { Support }\end{array}$ & Technical Support \\
\hline Diagnostic Category & ICD-9-CM Code & & & \\
\hline $\begin{array}{l}\text { HEALTH STATUS/HEALTH SERVICE } \\
\text { CONTACT }\end{array}$ & V01-V89 & 4 & 0 & 0 \\
\hline -Personal \& Family History & V10-V19 & 1 & 0 & 0 \\
\hline -Specific Procedures/Aftercare & V50-V59 & 3 & 0 & 0 \\
\hline
\end{tabular}

*Only those diagnostic categories and gender/job category combinations with at least one occurrence appear in this table. 
Nevada National Security Site 2010

Absence Data

Appendix I. Number of Diagnoses in Each Diagnostic Category by Gender and Job Category*

\begin{tabular}{|c|c|c|c|c|c|}
\hline & & \multicolumn{4}{|c|}{ Women } \\
\hline & & \multicolumn{3}{|c|}{ Job Category } & \multirow[b]{2}{*}{ TOTAL } \\
\hline & & Service & Security and Fire & Crafts & \\
\hline Diagnostic Category & ICD-9-CM Code & & & & \\
\hline $\begin{array}{l}\text { HEALTH STATUS/HEALTH SERVICE } \\
\text { CONTACT }\end{array}$ & V01-V89 & 0 & 0 & 0 & 4 \\
\hline -Personal \& Family History & V10-V19 & 0 & 0 & 0 & 1 \\
\hline -Specific Procedures/Aftercare & V50-V59 & 0 & 0 & 0 & 3 \\
\hline
\end{tabular}

\begin{tabular}{|c|c|c|c|c|c|c|c|}
\hline & \multicolumn{7}{|c|}{ Women } \\
\hline & \multicolumn{6}{|c|}{ Job Category } & \multirow[b]{2}{*}{ TOTAL } \\
\hline & Professional & $\begin{array}{c}\text { Administrative } \\
\text { Support }\end{array}$ & Technical Support & Service & Security and Fire & Crafts & \\
\hline Diagnostic Category & \multirow[b]{2}{*}{117} & \multirow[b]{2}{*}{67} & \multirow[b]{2}{*}{31} & \multirow[b]{2}{*}{19} & \multirow[b]{2}{*}{12} & \multirow[b]{2}{*}{21} & \multirow[b]{2}{*}{267} \\
\hline Total & & & & & & & \\
\hline
\end{tabular}

*Only those diagnostic categories and gender/job category combinations with at least one occurrence appear in this table. 
Nevada National Security Site 2010

Absence Data

Appendix I. Number of Diagnoses in Each Diagnostic Category by Gender and Job Category*

\begin{tabular}{|c|c|c|c|c|}
\hline & \multicolumn{3}{|c|}{ Men } \\
\hline & & \multicolumn{3}{|c|}{ Job Category } \\
\hline & & Professional & $\begin{array}{l}\text { Administrative } \\
\text { Support }\end{array}$ & Technical Support \\
\hline Diagnostic Category & ICD-9-CM Code & \multirow[b]{2}{*}{1} & \multirow[b]{2}{*}{0} & \multirow[b]{2}{*}{2} \\
\hline INFECTIOUS \& PARASITIC DISEASES (DIS) & 001-139 & & & \\
\hline -Intestinal Infectious Dis & \begin{tabular}{|c|}
$001-009$ \\
\end{tabular} & 0 & 0 & 0 \\
\hline -Other Bacterial Dis & $030-041$ & 0 & 0 & 1 \\
\hline -Viral Dis with Exanthem & $050-059$ & 0 & 0 & 0 \\
\hline -Other Viral Dis \& Chlamydiae & 070-079 & 0 & 0 & 1 \\
\hline -Other Infections \& Parasitic Dis & $130-136$ & 1 & 0 & 0 \\
\hline MALIGNANT NEOPLASMS & $\begin{array}{l}140-208, \\
209.0-209.3 \\
230-234\end{array}$ & 4 & 0 & 0 \\
\hline -Lip, Oral Cavity, Pharynx & $140-149$ & 0 & 0 & 0 \\
\hline -Digestive \& Peritoneal & \begin{tabular}{|c|}
$150-159$ \\
\end{tabular} & 0 & 0 & 0 \\
\hline -Genitourinary & 179-189 & 3 & 0 & $\overline{0}$ \\
\hline -Other \& Unspecified Sites & 195-199, 209.7 & 0 & 0 & 0 \\
\hline -Lymphatic \& Hematopoietic & $200-208$ & 1 & 0 & 0 \\
\hline BENIGN \& UNCERTAIN NEOPLASMS & $\begin{array}{l}209.4-209.6, \\
210-229,235-239\end{array}$ & 2 & 0 & 0 \\
\hline ENDOCRINE/METABOLIC/IMMUNITY & $240-279$ & 8 & 0 & 1 \\
\hline -Other Endocrine Gland Dis & 249-259 & 5 & 0 & 0 \\
\hline -Other Metabolic \& Immunity Disorders & $270-279$ & 3 & 0 & 1 \\
\hline BLOOD \& BLOOD-FORMING ORGANS & $280-289$ & 3 & 0 & 2 \\
\hline MENTAL DISORDERS & $290-319$ & 1 & 0 & 0 \\
\hline -Non-Psychotic Disorders & $300-302,306-316$ & 1 & 0 & 0 \\
\hline NERVOUS SYSTEM (NS) \& SENSE ORGANS & $320-389$ & 6 & 0 & 4 \\
\hline -Inflammatory Dis of Central NS & $320-326$ & 0 & 0 & 1 \\
\hline -Other Disorders of Central NS & $340-349$ & 0 & 0 & 1 \\
\hline -Disorders of Peripheral NS & 350-359 & 3 & 0 & 1 \\
\hline -Disorders of Eye & $360-379$ & 2 & 0 & 1 \\
\hline -Diseases of Ear \& Mastoid & $380-389$ & 1 & 0 & 0 \\
\hline CIRCULATORY SYSTEM & $390-459$ & 23 & 0 & 6 \\
\hline -Hypertensive Dis & $401-405$ & 7 & 0 & 0 \\
\hline -Ischemic Heart Dis & $410-414$ & 3 & 0 & 2 \\
\hline -Other Heart Dis & $420-429$ & 10 & 0 & 3 \\
\hline -Cerebrovascular Dis & $430-438$ & 3 & 0 & 0 \\
\hline -Dis of Arteries \& Capillaries & $440-449$ & 0 & 0 & 0 \\
\hline -Dis of Veins, Lymphatics, Other & $451-459$ & 0 & 0 & 1 \\
\hline RESPIRATORY SYSTEM & $460-519$ & 7 & 1 & 5 \\
\hline -Acute Respiratory Infections & $460-466$ & 1 & 0 & 1 \\
\hline
\end{tabular}

(Continued)

*Only those diagnostic categories and gender/job category combinations with at least one occurrence appear in this table. 
Nevada National Security Site 2010

Absence Data

Appendix I. Number of Diagnoses in Each Diagnostic Category by Gender and Job Category*

\begin{tabular}{|c|c|c|c|c|c|}
\hline & & \multicolumn{4}{|c|}{ Men } \\
\hline & & \multicolumn{3}{|c|}{ Job Category } & \multirow[b]{2}{*}{ TOTAL } \\
\hline & & Service & Security and Fire & Crafts & \\
\hline Diagnostic Category & ICD-9-CM Code & \multirow[b]{2}{*}{0} & \multirow[b]{2}{*}{5} & \multirow[b]{2}{*}{4} & \multirow[b]{2}{*}{12} \\
\hline INFECTIOUS \& PARASITIC DISEASES (DIS) & 001-139 & & & & \\
\hline -Intestinal Infectious Dis & 001-009 & 0 & 1 & 0 & 1 \\
\hline -Other Bacterial Dis & 030-041 & 0 & 2 & 1 & 4 \\
\hline -Viral Dis with Exanthem & $050-059$ & 0 & 0 & 1 & 1 \\
\hline -Other Viral Dis \& Chlamydiae & 070-079 & 0 & 2 & 2 & 5 \\
\hline -Other Infections \& Parasitic Dis & $130-136$ & 0 & 0 & 0 & 1 \\
\hline MALIGNANT NEOPLASMS & $\begin{array}{l}140-208, \\
209.0-209.3, \\
230-234\end{array}$ & 1 & 0 & 2 & 7 \\
\hline -Lip, Oral Cavity, Pharynx & $140-149$ & 0 & 0 & 1 & 1 \\
\hline -Digestive \& Peritoneal & $150-159$ & 1 & 0 & 0 & 1 \\
\hline -Genitourinary & 179-189 & 0 & 0 & 0 & 3 \\
\hline -Other \& Unspecified Sites & 195-199, 209.7 & 0 & 0 & 1 & 1 \\
\hline -Lymphatic \& Hematopoietic & $200-208$ & 0 & 0 & 0 & 1 \\
\hline BENIGN \& UNCERTAIN NEOPLASMS & $\begin{array}{l}209.4-209.6, \\
210-229,235-239\end{array}$ & 0 & 0 & 0 & 2 \\
\hline ENDOCRINE/METABOLIC/IMMUNITY & $240-279$ & 0 & 1 & 2 & 12 \\
\hline -Other Endocrine Gland Dis & 249-259 & 0 & 0 & 1 & 6 \\
\hline -Other Metabolic \& Immunity Disorders & 270-279 & 0 & 1 & 1 & 6 \\
\hline BLOOD \& BLOOD-FORMING ORGANS & $280-289$ & 0 & 0 & 1 & 6 \\
\hline MENTAL DISORDERS & $290-319$ & 1 & 0 & 0 & 2 \\
\hline -Non-Psychotic Disorders & $300-302,306-316$ & 1 & 0 & 0 & 2 \\
\hline NERVOUS SYSTEM (NS) \& SENSE ORGANS & $320-389$ & 0 & 3 & 0 & 13 \\
\hline -Inflammatory Dis of Central NS & $320-326$ & 0 & 0 & 0 & 1 \\
\hline -Other Disorders of Central NS & $340-349$ & 0 & 0 & 0 & 1 \\
\hline -Disorders of Peripheral NS & 350-359 & 0 & 0 & 0 & 4 \\
\hline -Disorders of Eye & $360-379$ & 0 & 3 & 0 & 6 \\
\hline -Diseases of Ear \& Mastoid & 380-389 & 0 & 0 & 0 & 1 \\
\hline CIRCULATORY SYSTEM & $390-459$ & 2 & 1 & 10 & 42 \\
\hline -Hypertensive Dis & $401-405$ & 1 & 0 & 7 & 15 \\
\hline -Ischemic Heart Dis & $410-414$ & 0 & 0 & 0 & 5 \\
\hline -Other Heart Dis & $420-429$ & 0 & 0 & 2 & 15 \\
\hline -Cerebrovascular Dis & $430-438$ & 1 & 0 & 0 & 4 \\
\hline -Dis of Arteries \& Capillaries & $440-449$ & 0 & 0 & 1 & 1 \\
\hline -Dis of Veins, Lymphatics, Other & 451-459 & 0 & 1 & 0 & 2 \\
\hline RESPIRATORY SYSTEM & $460-519$ & 2 & 14 & 7 & 36 \\
\hline -Acute Respiratory Infections & $460-466$ & 0 & 5 & 1 & 8 \\
\hline
\end{tabular}

(Continued)

*Only those diagnostic categories and gender/job category combinations with at least one occurrence appear in this table. 
Nevada National Security Site 2010

Absence Data

Appendix I. Number of Diagnoses in Each Diagnostic Category by Gender and Job Category*

\begin{tabular}{|c|c|c|c|c|}
\hline & \multicolumn{3}{|c|}{ Men } \\
\hline & & \multicolumn{3}{|c|}{ Job Category } \\
\hline & & Professional & $\begin{array}{c}\text { Administrative } \\
\text { Support }\end{array}$ & Technical Support \\
\hline Diagnostic Category & ICD-9-CM Code & \multirow[b]{2}{*}{2} & \multirow[b]{2}{*}{1} & \multirow[b]{2}{*}{4} \\
\hline -Other Dis Upper Respiratory Tract & $470-478$ & & & \\
\hline -Pneumonia \& Influenza & $480-488$ & 2 & 0 & $\overline{0}$ \\
\hline -Chronic Obstructive Dis & $490-496$ & 1 & 0 & 0 \\
\hline -Other Respiratory Dis & $510-519$ & 1 & 0 & 0 \\
\hline DIGESTIVE SYSTEM & $520-579$ & 6 & 1 & 10 \\
\hline -Oral Cavity, Saliva Glands, Jaw & $520-529$ & 1 & 0 & 1 \\
\hline -Esophagus, Stomach, Duodenum & $530-538$ & 0 & 0 & 1 \\
\hline -Appendicitis & $540-543$ & 2 & 0 & 0 \\
\hline -Hernias & $550-553$ & 1 & 1 & 3 \\
\hline -Other Intestinal Dis & $560-569$ & 1 & 0 & 4 \\
\hline -Other Digestive Dis & $570-579$ & 1 & 0 & 1 \\
\hline GENITOURINARY SYSTEM & $580-629$ & 8 & 0 & 0 \\
\hline -Other Urinary Dis & \begin{tabular}{|l|}
$590-599$ \\
\end{tabular} & 6 & 0 & $\overline{0}$ \\
\hline -Male Genital Organ Dis & $600-608$ & 2 & 0 & $\overline{0}$ \\
\hline -Breast Disorders & $610-612$ & 0 & 0 & 0 \\
\hline SKIN AND SUBCUTANEOUS TISSUE & $680-709$ & 4 & 0 & 1 \\
\hline -Infections & $680-686$ & 2 & 0 & 1 \\
\hline -Other Inflammatory Conditions & $690-698$ & 0 & 0 & 0 \\
\hline -Other & 700-709 & 2 & 0 & 0 \\
\hline $\begin{array}{l}\text { MUSCULOSKELETAL \& CONNECTIVE } \\
\text { TISSUE }\end{array}$ & $710-739$ & 25 & 0 & 4 \\
\hline -Arthropathies & $710-719$ & 13 & 0 & 0 \\
\hline -Dorsopathies & $720-724$ & 7 & 0 & 3 \\
\hline -Rheumatism, Excluding Back & 725-729 & 5 & 0 & 1 \\
\hline CONGENITAL ANOMALIES & 740-759 & 0 & 0 & 0 \\
\hline $\begin{array}{l}\text { SYMPTOMS, SIGNS, \& ILL-DEFINED } \\
\text { CONDITIONS }\end{array}$ & 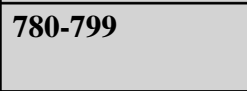 & 39 & 3 & 2 \\
\hline -Symptoms & $780-789$ & 37 & 2 & 2 \\
\hline -Non-Specific Abnormal Findings & $790-796$ & 2 & 1 & 0 \\
\hline INJURY \& POISONING & $800-999$ & 30 & 0 & 13 \\
\hline -Fracture - Neck, Trunk & $805-809$ & 1 & 0 & 4 \\
\hline -Fracture - Upper Limb & 810-819 & 1 & 0 & 0 \\
\hline -Fracture - Lower Limb & $820-829$ & 2 & 0 & 0 \\
\hline -Dislocation & $830-839$ & 7 & 0 & 1 \\
\hline -Sprains \& Strains - Back & 846-847 & 0 & 0 & 1 \\
\hline -Sprains \& Strains - Other & $840-845,848$ & 8 & 0 & 5 \\
\hline -Internal Injury - Thorax, Abdomen, Pelvis & $860-869$ & 2 & 0 & 0 \\
\hline
\end{tabular}

(Continued)

*Only those diagnostic categories and gender/job category combinations with at least one occurrence appear in this table. 
Nevada National Security Site 2010

Absence Data

Appendix I. Number of Diagnoses in Each Diagnostic Category by Gender and Job Category*

\begin{tabular}{|c|c|c|c|c|c|}
\hline & & \multicolumn{4}{|c|}{ Men } \\
\hline & & \multicolumn{3}{|c|}{ Job Category } & \multirow[b]{2}{*}{ TOTAL } \\
\hline & & Service & Security and Fire & Crafts & \\
\hline Diagnostic Category & ICD-9-CM Code & \multirow[b]{2}{*}{1} & \multirow[b]{2}{*}{5} & \multirow[b]{2}{*}{1} & \multirow[b]{2}{*}{14} \\
\hline -Other Dis Upper Respiratory Tract & $470-478$ & & & & \\
\hline -Pneumonia \& Influenza & $480-488$ & 0 & 1 & 0 & 3 \\
\hline -Chronic Obstructive Dis & $490-496$ & 1 & 2 & 4 & 8 \\
\hline -Other Respiratory Dis & $510-519$ & 0 & 1 & 1 & 3 \\
\hline DIGESTIVE SYSTEM & $520-579$ & 4 & 6 & 5 & 32 \\
\hline -Oral Cavity, Saliva Glands, Jaw & $520-529$ & 1 & 1 & 0 & 4 \\
\hline -Esophagus, Stomach, Duodenum & $530-538$ & 0 & 2 & 3 & 6 \\
\hline -Appendicitis & $540-543$ & 0 & 2 & 1 & 5 \\
\hline -Hernias & $550-553$ & 0 & 0 & 0 & 5 \\
\hline -Other Intestinal Dis & $560-569$ & 0 & 0 & 1 & 6 \\
\hline -Other Digestive Dis & $570-579$ & 3 & 1 & 0 & 6 \\
\hline GENITOURINARY SYSTEM & $580-629$ & 0 & 2 & 2 & 12 \\
\hline -Other Urinary Dis & $590-599$ & 0 & 0 & 2 & 8 \\
\hline -Male Genital Organ Dis & $600-608$ & 0 & 1 & 0 & 3 \\
\hline -Breast Disorders & $610-612$ & 0 & 1 & 0 & 1 \\
\hline SKIN AND SUBCUTANEOUS TISSUE & $680-709$ & 0 & 2 & 2 & 9 \\
\hline -Infections & $680-686$ & 0 & 1 & 2 & 6 \\
\hline -Other Inflammatory Conditions & $690-698$ & 0 & 1 & 0 & 1 \\
\hline -Other & 700-709 & 0 & 0 & 0 & 2 \\
\hline $\begin{array}{l}\text { MUSCULOSKELETAL \& CONNECTIVE } \\
\text { TISSUE }\end{array}$ & $710-739$ & 0 & 17 & 25 & 71 \\
\hline -Arthropathies & $710-719$ & 0 & 7 & 9 & 29 \\
\hline -Dorsopathies & $720-724$ & 0 & 7 & 12 & 29 \\
\hline -Rheumatism, Excluding Back & $725-729$ & 0 & 3 & 4 & 13 \\
\hline CONGENITAL ANOMALIES & 740-759 & 0 & 0 & 2 & 2 \\
\hline $\begin{array}{l}\text { SYMPTOMS, SIGNS, \& ILL-DEFINED } \\
\text { CONDITIONS }\end{array}$ & $780-799$ & 2 & 20 & 25 & 91 \\
\hline -Symptoms & $780-789$ & 2 & 17 & 21 & 81 \\
\hline -Non-Specific Abnormal Findings & $790-796$ & 0 & 3 & 4 & 10 \\
\hline INJURY \& POISONING & $800-999$ & 3 & 21 & 23 & 90 \\
\hline -Fracture - Neck, Trunk & 805-809 & 0 & 0 & 0 & 5 \\
\hline -Fracture - Upper Limb & 810-819 & 0 & 1 & 2 & 4 \\
\hline -Fracture - Lower Limb & 820-829 & 0 & 2 & 3 & 7 \\
\hline -Dislocation & 830-839 & 0 & 1 & 6 & 15 \\
\hline -Sprains \& Strains - Back & 846-847 & 2 & 4 & 2 & 9 \\
\hline -Sprains \& Strains - Other & $840-845,848$ & 0 & 9 & 2 & 24 \\
\hline -Internal Injury - Thorax, Abdomen, Pelvis & $860-869$ & 0 & 0 & 0 & 2 \\
\hline
\end{tabular}

(Continued)

*Only those diagnostic categories and gender/job category combinations with at least one occurrence appear in this table. 
Nevada National Security Site 2010

Absence Data

Appendix I. Number of Diagnoses in Each Diagnostic Category by Gender and Job Category*

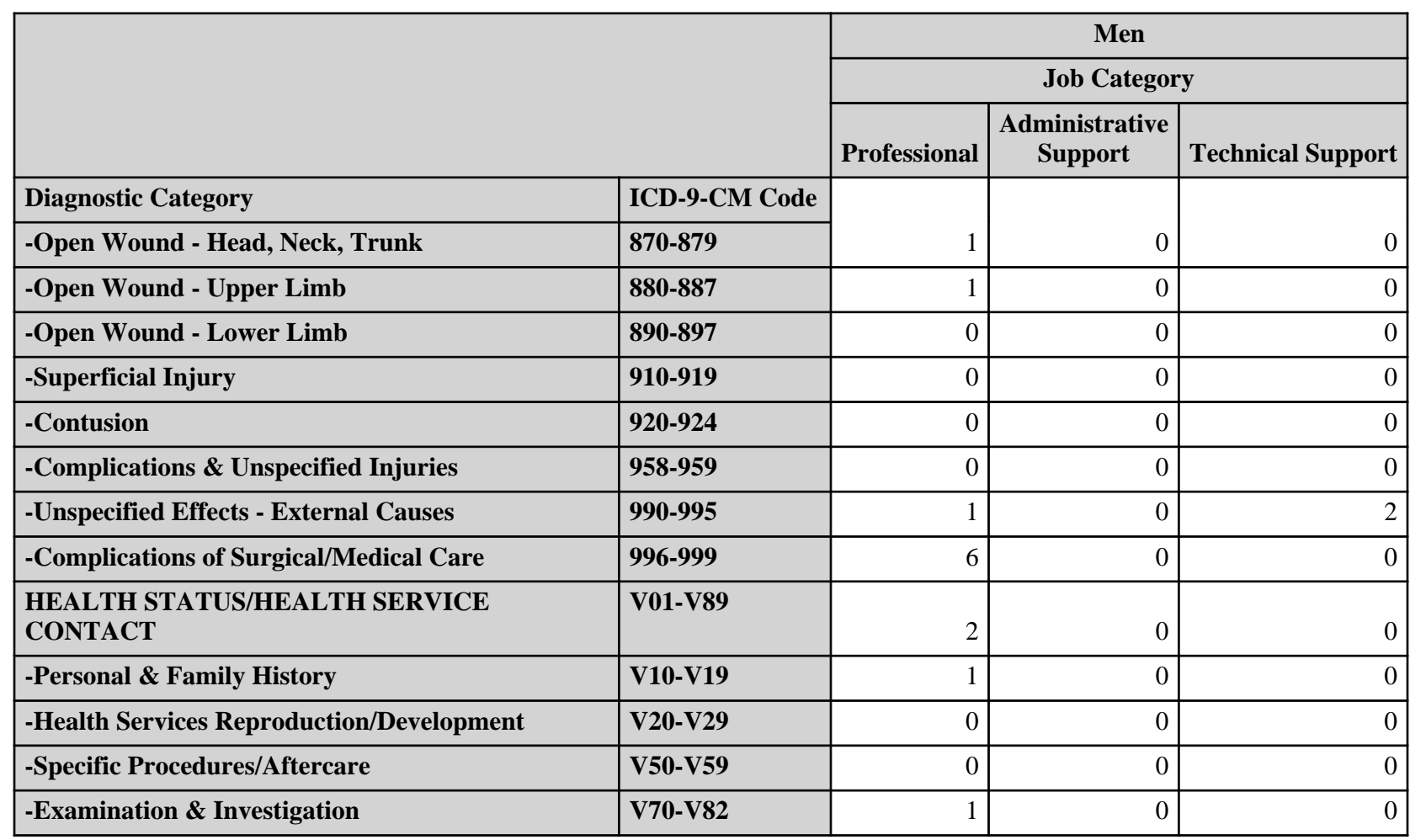

*Only those diagnostic categories and gender/job category combinations with at least one occurrence appear in this table. 
Nevada National Security Site 2010

Absence Data

Appendix I. Number of Diagnoses in Each Diagnostic Category by Gender and Job Category*

\begin{tabular}{|c|c|c|c|c|c|}
\hline & & \multicolumn{4}{|c|}{ Men } \\
\hline & & \multicolumn{3}{|c|}{ Job Category } & \multirow[b]{2}{*}{ TOTAL } \\
\hline & & Service & Security and Fire & Crafts & \\
\hline Diagnostic Category & ICD-9-CM Code & \multirow[b]{2}{*}{0} & \multirow[b]{2}{*}{0} & \multirow[b]{2}{*}{2} & \\
\hline -Open Wound - Head, Neck, Trunk & 870-879 & & & & 3 \\
\hline -Open Wound - Upper Limb & 880-887 & 0 & 0 & 0 & 1 \\
\hline -Open Wound - Lower Limb & $890-897$ & 0 & 1 & 0 & 1 \\
\hline -Superficial Injury & $910-919$ & 1 & 0 & 0 & 1 \\
\hline -Contusion & $920-924$ & 0 & 3 & 4 & 7 \\
\hline -Complications \& Unspecified Injuries & 958-959 & 0 & 0 & 1 & 1 \\
\hline -Unspecified Effects - External Causes & $990-995$ & 0 & 0 & 1 & 4 \\
\hline -Complications of Surgical/Medical Care & 996-999 & 0 & 0 & 0 & 6 \\
\hline $\begin{array}{l}\text { HEALTH STATUS/HEALTH SERVICE } \\
\text { CONTACT }\end{array}$ & V01-V89 & 0 & 8 & 0 & 10 \\
\hline -Personal \& Family History & V10-V19 & 0 & 2 & 0 & 3 \\
\hline -Health Services Reproduction/Development & V20-V29 & 0 & 5 & 0 & 5 \\
\hline -Specific Procedures/Aftercare & V50-V59 & 0 & 1 & 0 & 1 \\
\hline -Examination \& Investigation & V70-V82 & 0 & 0 & 0 & 1 \\
\hline
\end{tabular}

\begin{tabular}{|c|c|c|c|c|c|c|c|}
\hline & \multicolumn{7}{|c|}{ Men } \\
\hline & \multicolumn{6}{|c|}{ Job Category } & \multirow[b]{2}{*}{ TOTAI } \\
\hline & Professional & $\begin{array}{c}\text { Administrative } \\
\text { Support }\end{array}$ & Technical Support & Service & Security and Fire & Crafts & \\
\hline Diagnostic Category & \multirow[b]{2}{*}{169} & \multirow[b]{2}{*}{5} & \multirow[b]{2}{*}{50} & \multirow[b]{2}{*}{15} & \multirow[b]{2}{*}{100} & \multirow[b]{2}{*}{110} & \multirow[b]{2}{*}{449} \\
\hline Total & & & & & & & \\
\hline
\end{tabular}

*Only those diagnostic categories and gender/job category combinations with at least one occurrence appear in this table. 
Nevada National Security Site 2010

Absence Data

Appendix J. Total Number of Calendar Days Absent in Each Diagnostic Category by Gender and Job Category*

\begin{tabular}{|c|c|c|c|c|}
\hline & \multicolumn{3}{|c|}{ Women } \\
\hline & & \multicolumn{3}{|c|}{ Job Category } \\
\hline & & Professional & \begin{tabular}{|c|} 
Administrative \\
Support
\end{tabular} & Technical Support \\
\hline Diagnostic Category & ICD-9-CM Code & \multirow[b]{2}{*}{7} & \multirow[b]{2}{*}{108} & \multirow[b]{2}{*}{11} \\
\hline INFECTIOUS \& PARASITIC DISEASES (DIS) & 001-139 & & & \\
\hline MALIGNANT NEOPLASMS & $\begin{array}{l}140-208, \\
209.0-209.3 \\
230-234\end{array}$ & 0 & 88 & 0 \\
\hline BENIGN \& UNCERTAIN NEOPLASMS & $\begin{array}{l}209.4-209.6 \\
210-229,235-239\end{array}$ & 118 & 0 & 21 \\
\hline ENDOCRINE/METABOLIC/IMMUNITY & 240-279 & 74 & 59 & 14 \\
\hline MENTAL DISORDERS & $290-319$ & 25 & 5 & 0 \\
\hline NERVOUS SYSTEM (NS) \& SENSE ORGANS & 320-389 & 32 & 120 & 21 \\
\hline CIRCULATORY SYSTEM & $390-459$ & 50 & 136 & 0 \\
\hline RESPIRATORY SYSTEM & $460-519$ & 83 & 34 & 0 \\
\hline DIGESTIVE SYSTEM & 520-579 & 100 & 109 & 23 \\
\hline GENITOURINARY SYSTEM & $580-629$ & 140 & 37 & 121 \\
\hline PREGNANCY \& CHILDBIRTH & 630-679 & 21 & 0 & 0 \\
\hline SKIN AND SUBCUTANEOUS TISSUE & $680-709$ & 4 & 12 & 0 \\
\hline $\begin{array}{l}\text { MUSCULOSKELETAL \& CONNECTIVE } \\
\text { TISSUE }\end{array}$ & 710-739 & 497 & 252 & 147 \\
\hline $\begin{array}{l}\text { SYMPTOMS, SIGNS, \& ILL-DEFINED } \\
\text { CONDITIONS }\end{array}$ & 780-799 & 111 & 126 & 11 \\
\hline INJURY \& POISONING & $800-999$ & 206 & 181 & 137 \\
\hline
\end{tabular}

\footnotetext{
*Absences with >1 ICD-9-CM code in the same diagnostic category were counted only once. Only those diagnostic categories and gender/job
} category combinations with at least one occurrence appear in this table. 
Nevada National Security Site 2010

Absence Data

Appendix J. Total Number of Calendar Days Absent in Each Diagnostic Category by Gender and Job Category*

\begin{tabular}{|c|c|c|c|c|c|}
\hline & & \multicolumn{4}{|c|}{ Women } \\
\hline & & \multicolumn{3}{|c|}{ Job Category } & \multirow[b]{2}{*}{ TOTAI } \\
\hline & & Service & Security and Fire & Crafts & \\
\hline Diagnostic Category & ICD-9-CM Code & \multirow[b]{2}{*}{0} & \multirow[b]{2}{*}{2} & \multirow[b]{2}{*}{0} & \multirow[b]{2}{*}{128} \\
\hline INFECTIOUS \& PARASITIC DISEASES (DIS) & 001-139 & & & & \\
\hline MALIGNANT NEOPLASMS & $\begin{array}{l}140-208, \\
209.0-209.3 \\
230-234\end{array}$ & 0 & 0 & 28 & 116 \\
\hline BENIGN \& UNCERTAIN NEOPLASMS & $\begin{array}{l}209.4-209.6 \\
210-229,235-239\end{array}$ & 0 & 42 & 0 & 181 \\
\hline ENDOCRINE/METABOLIC/IMMUNITY & $240-279$ & 0 & 0 & 0 & 147 \\
\hline MENTAL DISORDERS & $290-319$ & 6 & 1 & 0 & 37 \\
\hline NERVOUS SYSTEM (NS) \& SENSE ORGANS & $320-389$ & 0 & 0 & 90 & 263 \\
\hline CIRCULATORY SYSTEM & $390-459$ & 0 & 0 & 259 & 445 \\
\hline RESPIRATORY SYSTEM & $460-519$ & 6 & 15 & 48 & 186 \\
\hline DIGESTIVE SYSTEM & $520-579$ & 7 & 0 & 0 & 239 \\
\hline GENITOURINARY SYSTEM & $580-629$ & 21 & 0 & 0 & 319 \\
\hline PREGNANCY \& CHILDBIRTH & $630-679$ & 0 & 0 & 0 & 21 \\
\hline SKIN AND SUBCUTANEOUS TISSUE & $680-709$ & 0 & 0 & 0 & 16 \\
\hline $\begin{array}{l}\text { MUSCULOSKELETAL \& CONNECTIVE } \\
\text { TISSUE }\end{array}$ & $710-739$ & 36 & 88 & 14 & 1,034 \\
\hline $\begin{array}{l}\text { SYMPTOMS, SIGNS, \& ILL-DEFINED } \\
\text { CONDITIONS }\end{array}$ & $780-799$ & 13 & 3 & 254 & 518 \\
\hline INJURY \& POISONING & 800-999 & 0 & 88 & 76 & 688 \\
\hline
\end{tabular}

\footnotetext{
*Absences with >1 ICD-9-CM code in the same diagnostic category were counted only once. Only those diagnostic categories and gender/job category combinations with at least one occurrence appear in this table.
} 
Nevada National Security Site 2010

Absence Data

Appendix J. Total Number of Calendar Days Absent in Each Diagnostic Category by Gender and Job Category*

\begin{tabular}{|c|c|c|c|c|}
\hline & \multicolumn{3}{|c|}{ Men } \\
\hline & & \multicolumn{3}{|c|}{ Job Category } \\
\hline & & Professional & \begin{tabular}{|c|} 
Administrative \\
Support
\end{tabular} & Technical Support \\
\hline Diagnostic Category & ICD-9-CM Code & \multirow[b]{2}{*}{11} & \multirow[b]{2}{*}{0} & \multirow[b]{2}{*}{30} \\
\hline INFECTIOUS \& PARASITIC DISEASES (DIS) & 001-139 & & & \\
\hline MALIGNANT NEOPLASMS & $\begin{array}{l}\text { 140-208, } \\
209.0-209.3 \\
230-234\end{array}$ & 215 & 0 & 0 \\
\hline BENIGN \& UNCERTAIN NEOPLASMS & $\begin{array}{l}\text { 209.4-209.6, } \\
210-229,235-239\end{array}$ & 76 & 0 & 0 \\
\hline ENDOCRINE/METABOLIC/IMMUNITY & 240-279 & 141 & 0 & 105 \\
\hline BLOOD \& BLOOD-FORMING ORGANS & 280-289 & 47 & 0 & 16 \\
\hline MENTAL DISORDERS & $290-319$ & 4 & 0 & 0 \\
\hline NERVOUS SYSTEM (NS) \& SENSE ORGANS & 320-389 & 51 & 0 & 153 \\
\hline CIRCULATORY SYSTEM & 390-459 & 239 & 0 & 176 \\
\hline RESPIRATORY SYSTEM & $460-519$ & 65 & 1 & 21 \\
\hline DIGESTIVE SYSTEM & 520-579 & 63 & 21 & 182 \\
\hline GENITOURINARY SYSTEM & 580-629 & 56 & 0 & 0 \\
\hline SKIN AND SUBCUTANEOUS TISSUE & $680-709$ & 56 & 0 & 23 \\
\hline $\begin{array}{l}\text { MUSCULOSKELETAL \& CONNECTIVE } \\
\text { TISSUE }\end{array}$ & 710-739 & 504 & 0 & 55 \\
\hline CONGENITAL ANOMALIES & 740-759 & 0 & 0 & 0 \\
\hline $\begin{array}{l}\text { SYMPTOMS, SIGNS, \& ILL-DEFINED } \\
\text { CONDITIONS }\end{array}$ & 780-799 & 335 & 22 & 28 \\
\hline INJURY \& POISONING & $800-999$ & 517 & 0 & 315 \\
\hline
\end{tabular}

\footnotetext{
*Absences with >1 ICD-9-CM code in the same diagnostic category were counted only once. Only those diagnostic categories and gender/job category combinations with at least one occurrence appear in this table.
} 
Nevada National Security Site 2010

Absence Data

Appendix J. Total Number of Calendar Days Absent in Each Diagnostic Category by Gender and Job Category*

\begin{tabular}{|c|c|c|c|c|c|}
\hline & & \multicolumn{4}{|c|}{ Men } \\
\hline & & \multicolumn{3}{|c|}{ Job Category } & \multirow[b]{2}{*}{ TOTAL } \\
\hline & & Service & Security and Fire & Crafts & \\
\hline Diagnostic Category & ICD-9-CM Code & \multirow[b]{2}{*}{0} & \multirow[b]{2}{*}{37} & \multirow[b]{2}{*}{31} & \multirow[b]{2}{*}{109} \\
\hline INFECTIOUS \& PARASITIC DISEASES (DIS) & 001-139 & & & & \\
\hline MALIGNANT NEOPLASMS & $\begin{array}{l}\text { 140-208, } \\
209.0-209.3 \\
230-234\end{array}$ & 61 & 0 & 3 & 279 \\
\hline BENIGN \& UNCERTAIN NEOPLASMS & \begin{tabular}{|l|}
$209.4-209.6$ \\
$210-229$, \\
\end{tabular} & 0 & 0 & 0 & 76 \\
\hline ENDOCRINE/METABOLIC/IMMUNITY & $240-279$ & 0 & 6 & 33 & 285 \\
\hline BLOOD \& BLOOD-FORMING ORGANS & $280-289$ & 0 & 0 & 4 & 67 \\
\hline MENTAL DISORDERS & 290-319 & 5 & 0 & 0 & 9 \\
\hline NERVOUS SYSTEM (NS) \& SENSE ORGANS & 320-389 & 0 & 62 & 0 & 266 \\
\hline CIRCULATORY SYSTEM & $390-459$ & 25 & 4 & 279 & 723 \\
\hline RESPIRATORY SYSTEM & $460-519$ & 5 & 51 & 41 & 184 \\
\hline DIGESTIVE SYSTEM & $520-579$ & 40 & 85 & 31 & 422 \\
\hline GENITOURINARY SYSTEM & $\begin{array}{l}580-629 \\
\end{array}$ & 0 & 18 & 6 & 80 \\
\hline SKIN AND SUBCUTANEOUS TISSUE & $680-709$ & 0 & 11 & 23 & 113 \\
\hline $\begin{array}{l}\text { MUSCULOSKELETAL \& CONNECTIVE } \\
\text { TISSUE }\end{array}$ & 710-739 & 0 & 568 & 533 & 1,660 \\
\hline CONGENITAL ANOMALIES & 740-759 & 0 & 0 & 138 & 138 \\
\hline $\begin{array}{l}\text { SYMPTOMS, SIGNS, \& ILL-DEFINED } \\
\text { CONDITIONS }\end{array}$ & 780-799 & 6 & 68 & 171 & 630 \\
\hline INJURY \& POISONING & $800-999$ & 1 & 378 & 911 & 2,122 \\
\hline
\end{tabular}

\footnotetext{
*Absences with >1 ICD-9-CM code in the same diagnostic category were counted only once. Only those diagnostic categories and gender/job
} category combinations with at least one occurrence appear in this table. 
Nevada National Security Site 2010

Absence Data

Appendix K. Age-Adjusted Illness and Injury Rates by Diagnostic Category*

Part 1. Men

\begin{tabular}{|c|c|c|c|c|c|}
\hline & & $\begin{array}{l}\text { Number of } \\
\text { Diagnoses }\end{array}$ & $\begin{array}{c}\text { Age-Adjusted } \\
\text { Rate per } 1,000 * *\end{array}$ & $\begin{array}{c}\text { Lower } 95 \% \\
\text { Confidence } \\
\text { Limit per 1,000 }\end{array}$ & $\begin{array}{c}\text { Upper } 95 \% \\
\text { Confidence } \\
\text { Limit per 1,000 }\end{array}$ \\
\hline Diagnostic Category & ICD-9-CM Code & & & & \\
\hline INFECTIOUS \& PARASITIC DISEASES (DIS) & 001-139 & 12 & 4.2 & 2.0 & 8.5 \\
\hline -Intestinal Infectious Dis & 001-009 & 1 & 0.2 & 0.0 & 1.2 \\
\hline -Other Bacterial Dis & 030-041 & 4 & 1.5 & 0.5 & 4.2 \\
\hline -Viral Dis with Exanthem & 050-059 & 1 & 0.2 & 0.0 & 1.3 \\
\hline -Other Viral Dis \& Chlamydiae & 070-079 & 5 & 2.1 & 0.7 & 6.8 \\
\hline -Other Infections \& Parasitic Dis & $130-136$ & 1 & 0.2 & 0.0 & 1.2 \\
\hline MALIGNANT NEOPLASMS & $\begin{array}{l}140-208, \\
209.0-209.3 \\
230-234\end{array}$ & 7 & 1.2 & 0.6 & 2.6 \\
\hline -Lip, Oral Cavity, Pharynx & $140-149$ & 1 & 0.2 & 0.0 & 1.2 \\
\hline -Digestive \& Peritoneal & $150-159$ & 1 & 0.2 & 0.0 & 1.3 \\
\hline -Genitourinary & 179-189 & 3 & 0.5 & 0.2 & 1.6 \\
\hline -Other \& Unspecified Sites & 195-199, 209.7 & 1 & 0.2 & 0.0 & 1.2 \\
\hline -Lymphatic \& Hematopoietic & $200-208$ & 1 & 0.2 & 0.0 & 1.2 \\
\hline BENIGN \& UNCERTAIN NEOPLASMS & $\begin{array}{l}209.4-209.6 \\
210-229,235-239\end{array}$ & 2 & 0.5 & 0.1 & 2.3 \\
\hline ENDOCRINE/METABOLIC/IMMUNITY & 240-279 & 12 & 2.6 & 1.4 & 4.8 \\
\hline -Other Endocrine Gland Dis & 249-259 & 6 & 1.2 & 0.5 & 2.8 \\
\hline -Other Metabolic \& Immunity Disorders & 270-279 & 6 & 1.4 & 0.6 & 3.4 \\
\hline BLOOD \& BLOOD-FORMING ORGANS & 280-289 & 6 & 1.2 & 0.5 & 2.8 \\
\hline MENTAL DISORDERS & 290-319 & 2 & 1.0 & 0.2 & 3.9 \\
\hline -Non-Psychotic Disorders & $300-302,306-316$ & 2 & 1.0 & 0.2 & 3.9 \\
\hline NERVOUS SYSTEM (NS) \& SENSE ORGANS & 320-389 & 13 & 3.1 & 1.7 & 5.6 \\
\hline -Inflammatory Dis of Central NS & $320-326$ & 1 & 0.2 & 0.0 & 1.3 \\
\hline -Other Disorders of Central NS & $340-349$ & 1 & 0.2 & 0.0 & 1.2 \\
\hline -Disorders of Peripheral NS & 350-359 & 4 & 0.9 & 0.3 & 2.5 \\
\hline -Disorders of Eye & $360-379$ & 6 & 1.4 & 0.6 & 3.3 \\
\hline -Diseases of Ear \& Mastoid & $380-389$ & 1 & 0.5 & 0.1 & 3.5 \\
\hline CIRCULATORY SYSTEM & $390-459$ & 42 & 8.0 & 5.9 & 11.0 \\
\hline -Hypertensive Dis & $401-405$ & 15 & 3.0 & 1.8 & 5.1 \\
\hline -Ischemic Heart Dis & $410-414$ & 5 & 0.9 & 0.4 & 2.1 \\
\hline -Other Heart Dis & $420-429$ & 15 & 2.6 & 1.6 & 4.4 \\
\hline -Cerebrovascular Dis & $430-438$ & 4 & 0.7 & 0.3 & 1.9 \\
\hline -Dis of Arteries \& Capillaries & $440-449$ & 1 & 0.2 & 0.0 & 1.2 \\
\hline -Dis of Veins, Lymphatics, Other & 451-459 & 2 & 0.7 & 0.1 & 3.1 \\
\hline RESPIRATORY SYSTEM & $460-519$ & 36 & 12.9 & 8.6 & 19.3 \\
\hline
\end{tabular}

(Continued)

* Only those diagnostic categories with at least one occurrence appear in this table.

**Standardized to age distribution of 2000 U.S. population. 
Nevada National Security Site 2010

Absence Data

Appendix K. Age-Adjusted Illness and Injury Rates by Diagnostic Category*

Part 1. Men

\begin{tabular}{|c|c|c|c|c|c|}
\hline & & $\begin{array}{l}\text { Number of } \\
\text { Diagnoses }\end{array}$ & \begin{tabular}{|} 
Age-Adjusted \\
Rate per $1,000^{* * *}$
\end{tabular} & \begin{tabular}{|c|} 
Lower 95\% \\
Confidence \\
Limit per 1,000
\end{tabular} & $\begin{array}{c}\text { Upper } 95 \% \\
\text { Confidence } \\
\text { Limit per 1,000 }\end{array}$ \\
\hline Diagnostic Category & ICD-9-CM Code & & & & \\
\hline -Acute Respiratory Infections & $460-466$ & 8 & 3.0 & 1.4 & 6.3 \\
\hline -Other Dis Upper Respiratory Tract & $470-478$ & 14 & 5.5 & 2.8 & 10.8 \\
\hline -Pneumonia \& Influenza & $480-488$ & 3 & 0.7 & 0.2 & 2.3 \\
\hline -Chronic Obstructive Dis & $490-496$ & 8 & 2.9 & 1.2 & 7.1 \\
\hline -Other Respiratory Dis & $510-519$ & 3 & 0.8 & 0.2 & 3.0 \\
\hline DIGESTIVE SYSTEM & 520-579 & 32 & 10.8 & 7.1 & 16.5 \\
\hline -Oral Cavity, Saliva Glands, Jaw & $520-529$ & 4 & 2.0 & 0.6 & 6.8 \\
\hline -Esophagus, Stomach, Duodenum & 530-538 & 6 & 2.8 & 1.1 & 7.3 \\
\hline -Appendicitis & $540-543$ & 5 & 1.8 & 0.7 & 4.7 \\
\hline -Hernias & $550-553$ & 5 & 1.1 & 0.4 & 2.7 \\
\hline -Other Intestinal Dis & 560-569 & 6 & 1.6 & 0.7 & 3.7 \\
\hline -Other Digestive Dis & 570-579 & 6 & 1.5 & 0.6 & 3.7 \\
\hline GENITOURINARY SYSTEM & $580-629$ & 12 & 3.0 & 1.4 & 6.8 \\
\hline -Other Urinary Dis & 590-599 & 8 & 1.4 & 0.7 & 2.8 \\
\hline -Male Genital Organ Dis & $600-608$ & 3 & 0.5 & 0.2 & 1.6 \\
\hline -Breast Disorders & $610-612$ & 1 & 1.1 & 0.2 & 7.9 \\
\hline SKIN AND SUBCUTANEOUS TISSUE & 680-709 & 9 & 3.1 & 1.3 & 7.2 \\
\hline -Infections & $680-686$ & 6 & 2.4 & 0.8 & 6.7 \\
\hline -Other Inflammatory Conditions & 690-698 & 1 & 0.4 & 0.0 & 2.5 \\
\hline -Other & 700-709 & 2 & 0.3 & 0.1 & 1.4 \\
\hline $\begin{array}{l}\text { MUSCULOSKELETAL \& CONNECTIVE } \\
\text { TISSUE }\end{array}$ & 710-739 & 71 & 20.0 & 15.2 & 26.4 \\
\hline -Arthropathies & 710-719 & 29 & 8.8 & 5.5 & 14.1 \\
\hline -Dorsopathies & 720-724 & 29 & 7.4 & 5.0 & 11.1 \\
\hline -Rheumatism, Excluding Back & 725-729 & 13 & 3.7 & 2.1 & 6.8 \\
\hline CONGENITAL ANOMALIES & 740-759 & 2 & 0.7 & 0.2 & 2.8 \\
\hline $\begin{array}{l}\text { SYMPTOMS, SIGNS, \& ILL-DEFINED } \\
\text { CONDITIONS }\end{array}$ & 780-799 & 91 & 28.9 & 22.4 & 37.2 \\
\hline -Symptoms & 780-789 & 81 & 26.3 & 20.1 & 34.4 \\
\hline -Non-Specific Abnormal Findings & 790-796 & 10 & 2.6 & 1.3 & 5.1 \\
\hline INJURY \& POISONING & $800-999$ & 90 & 30.9 & 23.9 & 39.9 \\
\hline -Fracture - Skull & $800-804$ & 5 & 1.4 & 0.6 & 3.5 \\
\hline -Fracture - Upper Limb & $810-819$ & 4 & 2.3 & 0.8 & 7.1 \\
\hline -Fracture - Lower Limb & $820-829$ & 7 & 2.5 & 0.9 & 6.8 \\
\hline -Dislocation & 830-839 & 15 & 4.3 & 2.5 & 7.4 \\
\hline -Sprains \& Strains - Back & 846-847 & 9 & 3.1 & 1.6 & 6.1 \\
\hline
\end{tabular}

(Continued)

* Only those diagnostic categories with at least one occurrence appear in this table.

**Standardized to age distribution of 2000 U.S. population. 
Nevada National Security Site 2010

Absence Data

Appendix K. Age-Adjusted IIIness and Injury Rates by Diagnostic Category*

Part 1. Men

\begin{tabular}{|c|c|c|c|c|c|}
\hline & & $\begin{array}{l}\text { Number of } \\
\text { Diagnoses }\end{array}$ & $\begin{array}{c}\text { Age-Adjusted } \\
\text { Rate per } 1,000 * *\end{array}$ & $\begin{array}{c}\text { Lower } 95 \% \\
\text { Confidence } \\
\text { Limit per } 1,000\end{array}$ & $\begin{array}{c}\text { Upper 95\% } \\
\text { Confidence } \\
\text { Limit per 1,000 }\end{array}$ \\
\hline Diagnostic Category & ICD-9-CM Code & \multirow[b]{2}{*}{24} & \multirow[b]{2}{*}{8.7} & \multirow[b]{2}{*}{5.2} & \multirow[b]{2}{*}{14.7} \\
\hline -Sprains \& Strains - Other & $840-845,848$ & & & & \\
\hline -Internal Injury - Thorax, Abdomen, Pelvis & $860-869$ & 2 & 0.3 & 0.1 & 1.4 \\
\hline -Open Wound - Head, Neck, Trunk & 870-879 & 5 & 2.3 & 0.8 & 6.9 \\
\hline -Superficial Injury & $910-919$ & 1 & 0.4 & 0.0 & 2.5 \\
\hline -Contusion & $920-924$ & 7 & 3.1 & 1.3 & 7.4 \\
\hline -Complications \& Unspecified Injuries & 958-959 & 1 & 0.4 & 0.0 & 2.5 \\
\hline -Unspecified Effects - External Causes & $990-995$ & 4 & 1.1 & 0.4 & 3.0 \\
\hline -Complications of Surgical/Medical Care & 996-999 & 6 & 1.1 & 0.5 & 2.4 \\
\hline Total & & 439 & 132.2 & 117.8 & 148.4 \\
\hline
\end{tabular}

*Only those diagnostic categories with at least one occurrence appear in this table.

**Standardized to age distribution of 2000 U.S. population. 
Nevada National Security Site 2010

Absence Data

Appendix K. Age-Adjusted Illness and Injury Rates by Diagnostic Category*

Part 2. Women

\begin{tabular}{|c|c|c|c|c|c|}
\hline & & $\begin{array}{l}\text { Number of } \\
\text { Diagnoses }\end{array}$ & $\begin{array}{c}\text { Age-Adjusted } \\
\text { Rate per 1,000** }\end{array}$ & $\begin{array}{c}\text { Lower } 95 \% \\
\text { Confidence } \\
\text { Limit per 1,000 }\end{array}$ & $\begin{array}{c}\text { Upper 95\% } \\
\text { Confidence } \\
\text { Limit per 1,000 }\end{array}$ \\
\hline Diagnostic Category & ICD-9-CM Code & & & & \\
\hline INFECTIOUS \& PARASITIC DISEASES (DIS) & 001-139 & 10 & 11.8 & 5.8 & 24.1 \\
\hline -Intestinal Infectious Dis & 001-009 & 2 & 2.5 & 0.6 & 10.6 \\
\hline -Other Bacterial Dis & 030-041 & 2 & 1.9 & 0.5 & 7.6 \\
\hline -Other Viral Dis \& Chlamydiae & 070-079 & 5 & 5.8 & 1.9 & 17.5 \\
\hline -Mycoses & 110-118 & 1 & 1.6 & 0.2 & 11.3 \\
\hline MALIGNANT NEOPLASMS & $\begin{array}{l}140-208, \\
209.0-209.3 \\
230-234\end{array}$ & 4 & 2.6 & 0.9 & 7.1 \\
\hline -Respiratory \& Intrathoracic & $160-165$ & 1 & 0.6 & 0.1 & 4.4 \\
\hline -Breast & 174-175 & 3 & 1.9 & 0.6 & 6.4 \\
\hline BENIGN \& UNCERTAIN NEOPLASMS & $\begin{array}{l}209.4-209.6 \\
210-229,235-239\end{array}$ & 5 & 2.9 & 1.2 & 7.3 \\
\hline ENDOCRINE/METABOLIC/IMMUNITY & 240-279 & 13 & 12.2 & 6.2 & 23.9 \\
\hline -Thyroid Gland Disorders & $240-246$ & 1 & 0.6 & 0.1 & 4.4 \\
\hline -Other Endocrine Gland Dis & 249-259 & 3 & 1.6 & 0.5 & 5.0 \\
\hline -Other Metabolic \& Immunity Disorders & 270-279 & 9 & 10.0 & 4.5 & 22.0 \\
\hline MENTAL DISORDERS & 290-319 & 5 & 9.1 & 3.7 & 22.4 \\
\hline -Psychoses & 290-299 & 1 & 1.6 & 0.2 & 11.3 \\
\hline -Non-Psychotic Disorders & $300-302,306-316$ & 3 & 5.9 & 1.8 & 19.0 \\
\hline -Drug Dependence & 304-305 & 1 & 1.6 & 0.2 & 11.3 \\
\hline NERVOUS SYSTEM (NS) \& SENSE ORGANS & $320-389$ & 14 & 10.6 & 6.0 & 18.6 \\
\hline -Disorders of Peripheral NS & 350-359 & 7 & 4.6 & 2.1 & 10.0 \\
\hline -Disorders of Eye & $360-379$ & 4 & 2.8 & 1.0 & 7.6 \\
\hline -Diseases of Ear \& Mastoid & 380-389 & 3 & 3.2 & 0.9 & 10.6 \\
\hline CIRCULATORY SYSTEM & $390-459$ & 14 & 9.0 & 5.3 & 15.5 \\
\hline -Hypertensive Dis & 401-405 & 5 & 3.4 & 1.3 & 8.5 \\
\hline -Ischemic Heart Dis & $410-414$ & 2 & 1.2 & 0.3 & 5.0 \\
\hline -Other Heart Dis & $420-429$ & 4 & 2.4 & 0.9 & 6.3 \\
\hline -Cerebrovascular Dis & $430-438$ & 1 & 0.6 & 0.1 & 4.4 \\
\hline -Dis of Arteries \& Capillaries & $440-449$ & 1 & 0.5 & 0.1 & 3.5 \\
\hline -Dis of Veins, Lymphatics, Other & $451-459$ & 1 & 0.9 & 0.1 & 6.7 \\
\hline RESPIRATORY SYSTEM & $460-519$ & 29 & 27.2 & 17.6 & 42.1 \\
\hline -Acute Respiratory Infections & $460-466$ & 6 & 10.1 & 4.1 & 24.5 \\
\hline -Other Dis Upper Respiratory Tract & $470-478$ & 11 & 7.2 & 3.9 & 13.3 \\
\hline -Pneumonia \& Influenza & $480-488$ & 3 & 2.7 & 0.7 & 9.8 \\
\hline -Chronic Obstructive Dis & $490-496$ & 8 & 6.7 & 3.1 & 14.8 \\
\hline
\end{tabular}

(Continued)

* Only those diagnostic categories with at least one occurrence appear in this table.

**Standardized to age distribution of 2000 U.S. population. 
Nevada National Security Site 2010

Absence Data

Appendix K. Age-Adjusted Illness and Injury Rates by Diagnostic Category*

Part 2. Women

\begin{tabular}{|c|c|c|c|c|c|}
\hline & & $\begin{array}{l}\text { Number of } \\
\text { Diagnoses }\end{array}$ & $\begin{array}{l}\text { Age-Adjusted } \\
\text { Rate per } 1,000 * *\end{array}$ & $\begin{array}{c}\text { Lower } 95 \% \\
\text { Confidence } \\
\text { Limit per 1,000 }\end{array}$ & $\begin{array}{c}\text { Upper } 95 \% \\
\text { Confidence } \\
\text { Limit per 1,000 }\end{array}$ \\
\hline Diagnostic Category & ICD-9-CM Code & \multirow[b]{2}{*}{1} & \multirow[b]{2}{*}{0.5} & \multirow[b]{2}{*}{0.1} & \multirow[b]{2}{*}{3.5} \\
\hline -Other Respiratory Dis & $510-519$ & & & & \\
\hline DIGESTIVE SYSTEM & $520-579$ & 25 & 19.0 & 12.5 & 28.7 \\
\hline -Oral Cavity, Saliva Glands, Jaw & $520-529$ & 3 & 2.8 & 0.9 & 8.8 \\
\hline -Esophagus, Stomach, Duodenum & \begin{tabular}{|l|}
$530-538$ \\
\end{tabular} & 1 & 0.9 & 0.1 & 6.7 \\
\hline -Appendicitis & $540-543$ & 3 & 2.7 & 0.7 & 9.8 \\
\hline -Hernias & \begin{tabular}{|l|}
$550-553$ \\
\end{tabular} & 2 & 1.6 & 0.4 & 6.5 \\
\hline -Enteritis, Colitis & $555-558$ & 3 & 1.9 & 0.6 & 6.4 \\
\hline -Other Intestinal Dis & $560-569$ & 3 & 1.9 & 0.6 & 6.4 \\
\hline -Other Digestive Dis & $570-579$ & 10 & 7.0 & 3.7 & 13.4 \\
\hline GENITOURINARY SYSTEM & $580-629$ & 24 & 24.6 & 16.2 & 37.5 \\
\hline -Other Urinary Dis & $590-599$ & 8 & 8.2 & 4.1 & 16.7 \\
\hline -Other Female Disorders & $617-629$ & 16 & 16.4 & 9.7 & 27.6 \\
\hline PREGNANCY \& CHILDBIRTH & $630-679$ & 1 & 1.6 & 0.2 & 11.3 \\
\hline -Ectopic \& Molar Pregnancy & $630-633$ & 1 & 1.6 & 0.2 & 11.3 \\
\hline SKIN AND SUBCUTANEOUS TISSUE & 680-709 & 2 & 1.6 & 0.4 & 6.5 \\
\hline -Other Inflammatory Conditions & $690-698$ & 1 & 0.6 & 0.1 & 4.4 \\
\hline -Other & 700-709 & 1 & 0.9 & 0.1 & 6.7 \\
\hline $\begin{array}{l}\text { MUSCULOSKELETAL \& CONNECTIVE } \\
\text { TISSUE }\end{array}$ & 710-739 & 44 & 29.9 & 21.5 & 41.5 \\
\hline -Arthropathies & 710-719 & 17 & 11.5 & 6.7 & 19.6 \\
\hline -Dorsopathies & $720-724$ & 17 & 13.0 & 7.7 & 22.0 \\
\hline -Rheumatism, Excluding Back & 725-729 & 10 & 5.4 & 2.9 & 10.3 \\
\hline $\begin{array}{l}\text { SYMPTOMS, SIGNS, \& ILL-DEFINED } \\
\text { CONDITIONS }\end{array}$ & \begin{tabular}{|l|}
$780-799$ \\
\end{tabular} & 41 & 47.7 & 33.0 & 68.9 \\
\hline -Symptoms & $780-789$ & 37 & 44.4 & 30.2 & 65.3 \\
\hline -Non-Specific Abnormal Findings & $790-796$ & 4 & 3.3 & 1.1 & 10.1 \\
\hline INJURY \& POISONING & $800-999$ & 32 & 34.5 & 23.1 & 51.5 \\
\hline -Fracture - Upper Limb & $810-819$ & 2 & 4.3 & 1.0 & 18.0 \\
\hline -Fracture - Lower Limb & $820-829$ & 3 & 2.6 & 0.7 & 9.7 \\
\hline -Dislocation & $830-839$ & 3 & 1.9 & 0.6 & 6.4 \\
\hline -Sprains \& Strains - Back & $846-847$ & 2 & 3.2 & 0.8 & 12.8 \\
\hline -Sprains \& Strains - Other & $840-845,848$ & 9 & 8.1 & 4.0 & 16.7 \\
\hline -Open Wound - Head, Neck, Trunk & $870-879$ & 1 & 0.6 & 0.1 & 4.4 \\
\hline -Late Effects of Accident & $905-909$ & 3 & 1.7 & 0.6 & 5.4 \\
\hline -Superficial Injury & 910-919 & 1 & 2.7 & 0.4 & 19.2 \\
\hline -Contusion & $920-924$ & 2 & 2.5 & 0.6 & $\overline{10.6}$ \\
\hline
\end{tabular}

(Continued)

* Only those diagnostic categories with at least one occurrence appear in this table.

**Standardized to age distribution of 2000 U.S. population. 
Nevada National Security Site 2010

Absence Data

Appendix K. Age-Adjusted IIIness and Injury Rates by Diagnostic Category*

Part 2. Women

\begin{tabular}{|c|c|c|c|c|c|}
\hline & & $\begin{array}{c}\text { Number of } \\
\text { Diagnoses }\end{array}$ & $\begin{array}{c}\text { Age-Adjusted } \\
\text { Rate per } 1,000 * *\end{array}$ & $\begin{array}{c}\text { Lower } 95 \% \\
\text { Confidence } \\
\text { Limit per 1,000 }\end{array}$ & $\begin{array}{c}\text { Upper } 95 \% \\
\text { Confidence } \\
\text { Limit per 1,000 }\end{array}$ \\
\hline Diagnostic Category & ICD-9-CM Code & \multirow[b]{2}{*}{6} & \multirow[b]{2}{*}{6.7} & \multirow[b]{2}{*}{2.8} & \multirow[b]{2}{*}{16.1} \\
\hline -Complications of Surgical/Medical Care & 996-999 & & & & \\
\hline Total & & 263 & 244.3 & 212.0 & 281.5 \\
\hline
\end{tabular}

*Only those diagnostic categories with at least one occurrence appear in this table.

**Standardized to age distribution of 2000 U.S. population. 
Nevada National Security Site 2010

Absence Data

Appendix K. Age-Adjusted Illness and Injury Rates by Diagnostic Category*

Part 3. Men and Women

\begin{tabular}{|c|c|c|c|c|c|}
\hline & & $\begin{array}{c}\text { Number of } \\
\text { Diagnoses }\end{array}$ & $\begin{array}{c}\text { Age-Adjusted } \\
\text { Rate per 1,000** }\end{array}$ & $\begin{array}{c}\text { Lower } 95 \% \\
\text { Confidence } \\
\text { Limit per 1,000 }\end{array}$ & $\begin{array}{c}\text { Upper 95\% } \\
\text { Confidence } \\
\text { Limit per 1,000 }\end{array}$ \\
\hline Diagnostic Category & ICD-9-CM Code & & & & \\
\hline INFECTIOUS \& PARASITIC DISEASES (DIS) & 001-139 & 22 & 6.2 & 3.7 & 10.2 \\
\hline -Intestinal Infectious Dis & 001-009 & 3 & 0.8 & 0.2 & 2.6 \\
\hline -Other Bacterial Dis & 030-041 & 6 & 1.7 & 0.7 & 3.8 \\
\hline -Viral Dis with Exanthem & 050-059 & 1 & 0.1 & 0.0 & 1.0 \\
\hline -Other Viral Dis \& Chlamydiae & 070-079 & 10 & 3.1 & 1.4 & 6.9 \\
\hline- Mycoses & 110-118 & 1 & 0.4 & 0.1 & 2.6 \\
\hline -Other Infections \& Parasitic Dis & $130-136$ & 1 & 0.1 & 0.0 & 0.9 \\
\hline MALIGNANT NEOPLASMS & $\begin{array}{l}140-208, \\
209.0-209.3 \\
230-234\end{array}$ & 11 & 1.6 & 0.9 & 3.0 \\
\hline -Lip, Oral Cavity, Pharynx & 140-149 & 1 & 0.1 & 0.0 & 0.9 \\
\hline -Digestive \& Peritoneal & $150-159$ & 1 & 0.1 & 0.0 & 1.0 \\
\hline -Respiratory \& Intrathoracic & $160-165$ & 1 & 0.1 & 0.0 & 1.0 \\
\hline -Breast & 174-175 & 3 & 0.5 & 0.2 & 1.7 \\
\hline -Genitourinary & 179-189 & 3 & 0.4 & 0.1 & 1.3 \\
\hline -Other \& Unspecified Sites & $195-199,209.7$ & 1 & 0.1 & 0.0 & 0.9 \\
\hline -Lymphatic \& Hematopoietic & $200-208$ & 1 & 0.1 & 0.0 & 0.9 \\
\hline BENIGN \& UNCERTAIN NEOPLASMS & $\begin{array}{l}209.4-209.6 \\
210-229,235-239\end{array}$ & 7 & 1.2 & 0.5 & 2.6 \\
\hline ENDOCRINE/METABOLIC/IMMUNITY & $240-279$ & 25 & 5.1 & 3.1 & 8.2 \\
\hline -Thyroid Gland Disorders & $240-246$ & 1 & 0.1 & 0.0 & 1.0 \\
\hline -Other Endocrine Gland Dis & 249-259 & 9 & 1.3 & 0.7 & 2.6 \\
\hline -Other Metabolic \& Immunity Disorders & $270-279$ & 15 & 3.6 & 1.9 & 6.8 \\
\hline BLOOD \& BLOOD-FORMING ORGANS & $280-289$ & 6 & 0.9 & 0.4 & 2.1 \\
\hline MENTAL DISORDERS & 290-319 & 7 & 3.0 & 1.4 & 6.6 \\
\hline -Psychoses & 290-299 & 1 & 0.4 & 0.1 & 2.6 \\
\hline -Non-Psychotic Disorders & $300-302,306-316$ & 5 & 2.3 & 0.9 & 5.8 \\
\hline -Drug Dependence & $304-305$ & 1 & 0.4 & 0.1 & 2.6 \\
\hline NERVOUS SYSTEM (NS) \& SENSE ORGANS & 320-389 & 27 & 5.0 & 3.3 & 7.5 \\
\hline -Inflammatory Dis of Central NS & $320-326$ & 1 & 0.1 & 0.0 & 1.0 \\
\hline -Other Disorders of Central NS & $340-349$ & 1 & 0.1 & 0.0 & 0.9 \\
\hline -Disorders of Peripheral NS & 350-359 & 11 & 1.8 & 1.0 & 3.4 \\
\hline -Disorders of Eye & $360-379$ & 10 & 1.7 & 0.9 & 3.3 \\
\hline -Diseases of Ear \& Mastoid & $380-389$ & 4 & 1.1 & 0.4 & 3.2 \\
\hline CIRCULATORY SYSTEM & $390-459$ & 56 & 8.4 & 6.4 & 11.1 \\
\hline -Hypertensive Dis & $401-405$ & 20 & 3.2 & 2.0 & 5.0 \\
\hline
\end{tabular}

(Continued)

* Only those diagnostic categories with at least one occurrence appear in this table.

**Standardized to age distribution of 2000 U.S. population. 
Nevada National Security Site 2010

Absence Data

Appendix K. Age-Adjusted Illness and Injury Rates by Diagnostic Category*

Part 3. Men and Women

\begin{tabular}{|c|c|c|c|c|c|}
\hline & & $\begin{array}{l}\text { Number of } \\
\text { Diagnoses }\end{array}$ & $\begin{array}{c}\text { Age-Adjusted } \\
\text { Rate per } 1,000 * *\end{array}$ & $\begin{array}{c}\text { Lower } 95 \% \\
\text { Confidence } \\
\text { Limit per 1,000 }\end{array}$ & $\begin{array}{c}\text { Upper } 95 \% \\
\text { Confidence } \\
\text { Limit per 1,000 }\end{array}$ \\
\hline Diagnostic Category & ICD-9-CM Code & \multirow[b]{2}{*}{7} & \multirow[b]{2}{*}{1.0} & \multirow[b]{2}{*}{0.5} & \multirow[b]{2}{*}{2.1} \\
\hline -Ischemic Heart Dis & $410-414$ & & & & \\
\hline -Other Heart Dis & $420-429$ & 19 & 2.5 & 1.6 & 4.0 \\
\hline -Cerebrovascular Dis & $430-438$ & 5 & 0.7 & 0.3 & 1.7 \\
\hline -Dis of Arteries \& Capillaries & $440-449$ & 2 & 0.3 & 0.1 & 1.0 \\
\hline -Dis of Veins, Lymphatics, Other & 451-459 & 3 & 0.8 & 0.2 & 2.6 \\
\hline RESPIRATORY SYSTEM & $460-519$ & 65 & 16.5 & 12.3 & 22.2 \\
\hline -Acute Respiratory Infections & $460-466$ & 14 & 5.0 & 2.7 & 9.0 \\
\hline -Other Dis Upper Respiratory Tract & $470-478$ & 25 & 5.9 & 3.6 & 9.6 \\
\hline -Pneumonia \& Influenza & $480-488$ & 6 & 1.2 & 0.5 & 2.8 \\
\hline -Chronic Obstructive Dis & $490-496$ & 16 & 3.7 & 2.1 & 6.8 \\
\hline -Other Respiratory Dis & $510-519$ & 4 & 0.8 & 0.3 & 2.3 \\
\hline DIGESTIVE SYSTEM & $520-579$ & 57 & 13.0 & 9.6 & 17.5 \\
\hline -Oral Cavity, Saliva Glands, Jaw & $520-529$ & 7 & 2.2 & 0.9 & 5.3 \\
\hline -Esophagus, Stomach, Duodenum & $530-538$ & 7 & 2.3 & 1.0 & 5.5 \\
\hline -Appendicitis & $540-543$ & 8 & 2.0 & 0.9 & 4.4 \\
\hline -Hernias & $550-553$ & 7 & 1.2 & 0.6 & 2.6 \\
\hline -Enteritis, Colitis & $555-558$ & 3 & 0.5 & 0.2 & 1.7 \\
\hline -Other Intestinal Dis & $560-569$ & 9 & 1.7 & 0.8 & 3.3 \\
\hline -Other Digestive Dis & $570-579$ & 16 & 3.0 & 1.8 & 5.1 \\
\hline GENITOURINARY SYSTEM & $580-629$ & 36 & 8.6 & 6.0 & 12.4 \\
\hline -Other Urinary Dis & $590-599$ & 16 & 3.2 & 1.9 & 5.4 \\
\hline -Male Genital Organ Dis & $600-608$ & 3 & 0.4 & 0.1 & 1.2 \\
\hline -Breast Disorders & $610-612$ & 1 & 0.8 & 0.1 & 5.6 \\
\hline -Other Female Disorders & $617-629$ & 16 & 4.2 & 2.5 & 7.0 \\
\hline PREGNANCY \& CHILDBIRTH & $630-679$ & 1 & 0.4 & 0.1 & 2.6 \\
\hline -Ectopic \& Molar Pregnancy & $630-633$ & 1 & 0.4 & 0.1 & 2.6 \\
\hline SKIN AND SUBCUTANEOUS TISSUE & 680-709 & 11 & 2.6 & 1.3 & 5.5 \\
\hline -Infections & $680-686$ & 6 & 1.7 & 0.6 & 4.8 \\
\hline -Other Inflammatory Conditions & 690-698 & 2 & 0.4 & 0.1 & 1.7 \\
\hline -Other & 700-709 & 3 & 0.5 & 0.2 & 1.7 \\
\hline $\begin{array}{l}\text { MUSCULOSKELETAL \& CONNECTIVE } \\
\text { TISSUE }\end{array}$ & $710-739$ & 115 & 22.6 & 18.3 & 27.9 \\
\hline -Arthropathies & $710-719$ & 46 & 9.4 & 6.6 & 13.4 \\
\hline -Dorsopathies & $720-724$ & 46 & 8.9 & 6.5 & 12.2 \\
\hline -Rheumatism, Excluding Back & 725-729 & 23 & 4.3 & 2.7 & 6.7 \\
\hline CONGENITAL ANOMALIES & $740-759$ & 2 & 0.5 & 0.1 & 2.1 \\
\hline
\end{tabular}

(Continued)

* Only those diagnostic categories with at least one occurrence appear in this table.

**Standardized to age distribution of 2000 U.S. population. 
Nevada National Security Site 2010

Absence Data

Appendix K. Age-Adjusted Illness and Injury Rates by Diagnostic Category*

Part 3. Men and Women

\begin{tabular}{|c|c|c|c|c|c|}
\hline & & $\begin{array}{l}\text { Number of } \\
\text { Diagnoses }\end{array}$ & $\begin{array}{c}\text { Age-Adjusted } \\
\text { Rate per 1,000** }\end{array}$ & $\begin{array}{c}\text { Lower } 95 \% \\
\text { Confidence } \\
\text { Limit per 1,000 }\end{array}$ & $\begin{array}{c}\text { Upper } 95 \% \\
\text { Confidence } \\
\text { Limit per 1,000 }\end{array}$ \\
\hline Diagnostic Category & ICD-9-CM Code & & & & \\
\hline $\begin{array}{l}\text { SYMPTOMS, SIGNS, \& ILL-DEFINED } \\
\text { CONDITIONS }\end{array}$ & $780-799$ & 132 & 33.9 & 27.5 & 41.8 \\
\hline -Symptoms & $780-789$ & 118 & 31.2 & 25.0 & 39.0 \\
\hline -Non-Specific Abnormal Findings & $790-796$ & 14 & 2.7 & 1.5 & 4.9 \\
\hline INJURY \& POISONING & $800-999$ & 122 & 31.4 & 25.4 & 38.8 \\
\hline -Fracture - Skull & $800-804$ & 5 & 1.0 & 0.4 & 2.6 \\
\hline -Fracture - Upper Limb & 810-819 & 6 & 2.8 & 1.2 & 6.9 \\
\hline -Fracture - Lower Limb & $820-829$ & 10 & 2.4 & 1.1 & 5.4 \\
\hline -Dislocation & 830-839 & 18 & 3.7 & 2.2 & 6.2 \\
\hline -Sprains \& Strains - Back & \begin{tabular}{|l|}
$846-847$ \\
\end{tabular} & 11 & 3.0 & 1.7 & 5.6 \\
\hline -Sprains \& Strains - Other & $840-845,848$ & 33 & 8.4 & 5.5 & 12.9 \\
\hline -Internal Injury - Thorax, Abdomen, Pelvis & $860-869$ & 2 & 0.3 & 0.1 & 1.0 \\
\hline -Open Wound - Head, Neck, Trunk & 870-879 & 6 & 1.8 & 0.7 & 5.0 \\
\hline -Late Effects of Accident & $905-909$ & 3 & 0.4 & 0.1 & 1.3 \\
\hline -Superficial Injury & $910-919$ & 2 & 1.0 & 0.2 & 4.9 \\
\hline -Contusion & $920-924$ & 9 & 2.8 & 1.3 & 6.0 \\
\hline -Complications \& Unspecified Injuries & 958-959 & 1 & 0.3 & 0.0 & 1.8 \\
\hline -Unspecified Effects - External Causes & $990-995$ & 4 & 0.8 & 0.3 & 2.2 \\
\hline -Complications of Surgical/Medical Care & 996-999 & 12 & 2.5 & 1.3 & 4.6 \\
\hline Total & & 702 & 160.9 & 147.2 & 175.9 \\
\hline
\end{tabular}

* Only those diagnostic categories with at least one occurrence appear in this table.

**Standardized to age distribution of 2000 U.S. population. 
Nevada National Security Site 2010

OSHA Data

Appendix L. Number of Workers with at Least One OSHA Event by Gender, Age, and Job Category*

\begin{tabular}{|c|c|c|c|c|c|c|c|c|c|c|c|}
\hline \multirow{3}{*}{ Job Category } & \multicolumn{5}{|c|}{ Women } & \multicolumn{5}{|c|}{ Men } & \multirow[b]{3}{*}{ TOTAL } \\
\hline & \multicolumn{4}{|c|}{ Age Group } & \multirow[b]{2}{*}{ TOTAL } & \multicolumn{4}{|c|}{ Age Group } & \multirow[b]{2}{*}{ TOTAL } & \\
\hline & $16-29$ & $30-39$ & $40-49$ & $50+$ & & $16-29$ & $30-39$ & $40-49$ & $50+$ & & \\
\hline Professional & 0 & 1 & 0 & 2 & 3 & 1 & 2 & 2 & 4 & 9 & 12 \\
\hline Administrative Support & 0 & 0 & 0 & 2 & 2 & 0 & 0 & 0 & 0 & 0 & 2 \\
\hline Technical Support & 1 & 1 & 0 & 3 & 5 & 3 & 4 & 5 & 5 & 17 & 22 \\
\hline Service & 0 & 0 & 0 & 2 & 2 & 0 & 0 & 0 & 1 & 1 & 3 \\
\hline Crafts & 0 & 0 & 1 & 0 & 1 & 0 & 0 & 5 & 6 & 11 & 12 \\
\hline TOTAL & 1 & 2 & 1 & 9 & 13 & 4 & 6 & 12 & 16 & 38 & 51 \\
\hline
\end{tabular}

*Only those job categories and gender/age combinations with at least one OSHA event appear in this table.

Appendix M. Total Number of Workdays Lost or with Restricted Activity from OSHA Events by Gender and Age

\begin{tabular}{|c|c|c|c|c|c|c|c|c|c|}
\hline \multirow[b]{2}{*}{ Age Group } & \multicolumn{3}{|c|}{ Women } & \multicolumn{3}{|c|}{ Men } & \multicolumn{3}{|c|}{ TOTAL } \\
\hline & $\begin{array}{l}\text { Number of } \\
\text { Events }\end{array}$ & $\begin{array}{c}\text { Days } \\
\text { Restricted }\end{array}$ & Days Lost & $\begin{array}{l}\text { Number of } \\
\text { Events }\end{array}$ & $\begin{array}{c}\text { Days } \\
\text { Restricted }\end{array}$ & Days Lost & $\begin{array}{l}\text { Number of } \\
\text { Events }\end{array}$ & $\begin{array}{c}\text { Days } \\
\text { Restricted }\end{array}$ & Days Lost \\
\hline $16-29$ & 1 & 16 & 24 & 4 & 7 & 3 & 5 & 23 & 27 \\
\hline $30-39$ & 2 & 0 & 0 & 6 & 2 & 81 & 8 & 2 & 81 \\
\hline $40-49$ & 1 & 0 & 63 & 12 & 93 & 218 & 13 & 93 & 281 \\
\hline $50+$ & 9 & 152 & 6 & 16 & 32 & 288 & 25 & 184 & 294 \\
\hline TOTAL & 13 & 168 & 93 & 38 & 134 & 590 & 51 & 302 & 683 \\
\hline
\end{tabular}

Appendix N. Total Number of Workdays Lost or with Restricted Activity from OSHA Events by Gender and Job Category*

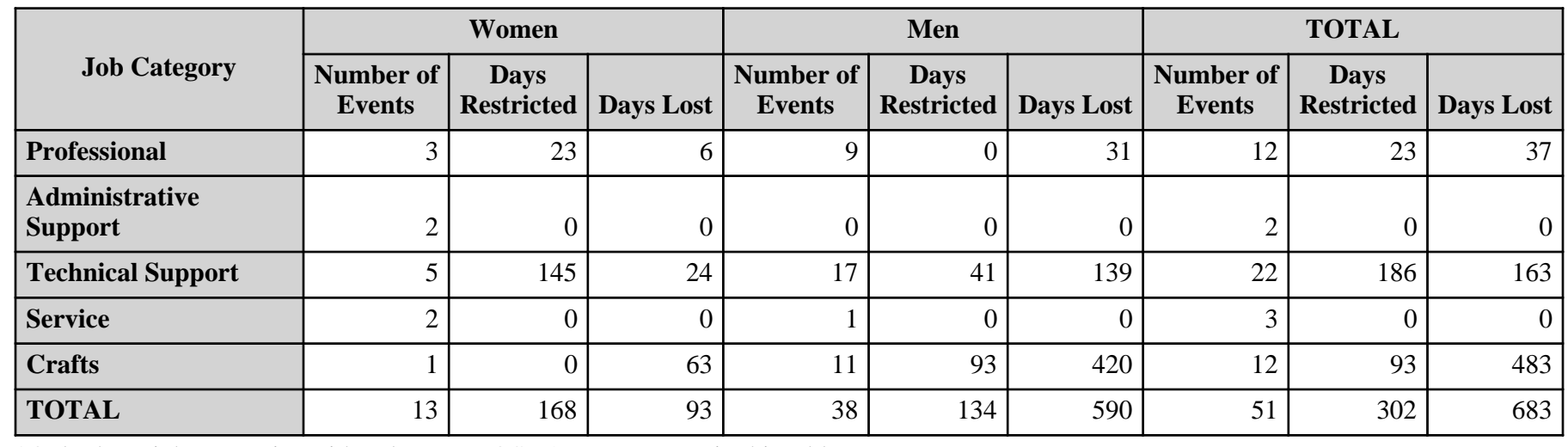

*Only those job categories with at least one OSHA event appear in this table. 
Nevada National Security Site 2010

OSHA Data

Appendix O. Number of Diagnoses in Each Diagnostic Category by Gender and Age*

\begin{tabular}{|c|c|c|c|c|c|c|}
\hline & & \multicolumn{5}{|c|}{ Women } \\
\hline & & \multicolumn{4}{|c|}{ Age Group } & \multirow[b]{2}{*}{ TOTAL } \\
\hline & & $16-29$ & 30 - 39 & $40-49$ & $50+$ & \\
\hline Diagnostic Category & ICD-9-CM code & \multirow[b]{2}{*}{0} & \multirow[b]{2}{*}{0} & \multirow[b]{2}{*}{0} & \multirow[b]{2}{*}{0} & \multirow[b]{2}{*}{0} \\
\hline NERVOUS SYSTEM (NS) \& SENSE ORGANS & $320-389$ & & & & & \\
\hline -Diseases of Ear \& Mastoid & $380-389$ & 0 & 0 & 0 & 0 & 0 \\
\hline RESPIRATORY SYSTEM & $460-519$ & 0 & 0 & 0 & 0 & 0 \\
\hline -Lung Dis from External Agents & $500-508$ & 0 & 0 & 0 & 0 & $\overline{0}$ \\
\hline $\begin{array}{l}\text { MUSCULOSKELETAL \& CONNECTIVE } \\
\text { TISSUE }\end{array}$ & $710-739$ & 0 & 4 & 0 & 5 & 9 \\
\hline -Arthropathies & $710-719$ & 0 & 2 & 0 & 4 & 6 \\
\hline -Dorsopathies & $720-724$ & 0 & 1 & 0 & 1 & 2 \\
\hline -Rheumatism, Excluding Back & $725-729$ & 0 & 1 & 0 & 0 & 1 \\
\hline $\begin{array}{l}\text { SYMPTOMS, SIGNS, \& ILL-DEFINED } \\
\text { CONDITIONS }\end{array}$ & 780-799 & 0 & 0 & 0 & 0 & 0 \\
\hline -Symptoms & 780-789 & 0 & 0 & 0 & 0 & 0 \\
\hline INJURY \& POISONING & 800-999 & 1 & 1 & 1 & 13 & 16 \\
\hline -Fracture - Upper Limb & 810-819 & 0 & 0 & 0 & 0 & 0 \\
\hline -Fracture - Lower Limb & $820-829$ & 0 & 0 & 0 & 2 & 2 \\
\hline -Dislocation & 830-839 & 0 & 0 & 0 & 1 & 1 \\
\hline -Sprains \& Strains - Back & 846-847 & 0 & 1 & 0 & 1 & 2 \\
\hline -Sprains \& Strains - Other & $840-845,848$ & 1 & 0 & 0 & 2 & 3 \\
\hline -Intracranial Injury & $850-854$ & 0 & 0 & 0 & 0 & 0 \\
\hline -Open Wound - Head, Neck, Trunk & 870-879 & 0 & 0 & 0 & 0 & 0 \\
\hline -Open Wound - Upper Limb & $880-887$ & 0 & 0 & 0 & 0 & 0 \\
\hline -Open Wound - Lower Limb & 890-897 & 0 & 0 & 0 & 1 & 1 \\
\hline -Superficial Injury & 910-919 & 0 & 0 & 0 & 1 & 1 \\
\hline -Contusion & \begin{tabular}{|l|}
$920-924$ \\
\end{tabular} & 0 & 0 & 0 & 0 & 0 \\
\hline -Burns & 940-949 & 0 & 0 & 0 & 0 & 0 \\
\hline -Complications \& Unspecified Injuries & 958-959 & 0 & 0 & 1 & 5 & 6 \\
\hline
\end{tabular}

*Only those diagnostic categories and gender/age combinations with at least one OSHA event appear in this table. 
Nevada National Security Site 2010

OSHA Data

Appendix O. Number of Diagnoses in Each Diagnostic Category by Gender and Age*

\begin{tabular}{|c|c|c|c|c|c|c|c|}
\hline & & \multicolumn{5}{|c|}{ Men } & \multirow[b]{3}{*}{ TOTAL } \\
\hline & & \multicolumn{4}{|c|}{ Age Group } & \multirow[b]{2}{*}{ TOTAL } & \\
\hline & & $16-29$ & 30 - 39 & $40-49$ & $50+$ & & \\
\hline Diagnostic Category & ICD-9-CM code & & & & & & \\
\hline NERVOUS SYSTEM (NS) \& SENSE ORGANS & $320-389$ & 0 & 0 & 0 & 1 & 1 & 1 \\
\hline -Diseases of Ear \& Mastoid & $380-389$ & 0 & 0 & 0 & 1 & 1 & 1 \\
\hline RESPIRATORY SYSTEM & $460-519$ & 0 & 0 & 1 & 0 & 1 & 1 \\
\hline -Lung Dis from External Agents & $500-508$ & 0 & 0 & 1 & 0 & 1 & 1 \\
\hline $\begin{array}{l}\text { MUSCULOSKELETAL \& CONNECTIVE } \\
\text { TISSUE }\end{array}$ & 710-739 & 4 & 1 & 8 & 3 & 16 & 25 \\
\hline -Arthropathies & $710-719$ & 2 & 0 & 4 & 2 & 8 & 14 \\
\hline -Dorsopathies & $720-724$ & 2 & 1 & 2 & 1 & 6 & 8 \\
\hline -Rheumatism, Excluding Back & $725-729$ & 0 & 0 & 2 & 0 & 2 & 3 \\
\hline $\begin{array}{l}\text { SYMPTOMS, SIGNS, \& ILL-DEFINED } \\
\text { CONDITIONS }\end{array}$ & $780-799$ & 0 & 0 & 0 & 1 & 1 & 1 \\
\hline -Symptoms & 780-789 & 0 & 0 & 0 & 1 & 1 & 1 \\
\hline INJURY \& POISONING & $800-999$ & 4 & 6 & 11 & 18 & 39 & 55 \\
\hline -Fracture - Upper Limb & 810-819 & 0 & 0 & 1 & 1 & 2 & 2 \\
\hline -Fracture - Lower Limb & $820-829$ & 0 & 0 & 0 & 0 & 0 & 2 \\
\hline -Dislocation & 830-839 & 0 & 0 & 0 & 0 & 0 & 1 \\
\hline -Sprains \& Strains - Back & 846-847 & 2 & 1 & 3 & 3 & 9 & 11 \\
\hline -Sprains \& Strains - Other & $840-845,848$ & 0 & 2 & 3 & 5 & 10 & 13 \\
\hline -Intracranial Injury & $850-854$ & 0 & 0 & 0 & 1 & 1 & 1 \\
\hline -Open Wound - Head, Neck, Trunk & 870-879 & 0 & 1 & 0 & 3 & 4 & 4 \\
\hline -Open Wound - Upper Limb & $880-887$ & 1 & 1 & 1 & 0 & 3 & 3 \\
\hline -Open Wound - Lower Limb & \begin{tabular}{|l|}
$890-897$ \\
\end{tabular} & 0 & 0 & 0 & 0 & 0 & 1 \\
\hline -Superficial Injury & 910-919 & 0 & 0 & 0 & 2 & 2 & 3 \\
\hline -Contusion & \begin{tabular}{|l|}
$920-924$ \\
\end{tabular} & 0 & 0 & 1 & 1 & 2 & 2 \\
\hline -Burns & $940-949$ & 0 & 1 & 0 & 0 & 1 & 1 \\
\hline -Complications \& Unspecified Injuries & 958-959 & 1 & 0 & 2 & 2 & 5 & 11 \\
\hline
\end{tabular}

\begin{tabular}{|c|c|c|c|c|c|c|c|c|c|c|c|}
\hline & \multicolumn{5}{|c|}{ Women } & \multicolumn{5}{|c|}{ Men } & \multirow[b]{3}{*}{ TOTAL } \\
\hline & \multicolumn{4}{|c|}{ Age Group } & \multirow[b]{2}{*}{ TOTAL } & \multicolumn{4}{|c|}{ Age Group } & \multirow[b]{2}{*}{ TOTAL } & \\
\hline & $16-29$ & $30-39$ & $40-49$ & $50+$ & & $16-29$ & $30-39$ & $40-49$ & $50+$ & & \\
\hline Diagnostic Category & \multirow[b]{2}{*}{1} & \multirow[b]{2}{*}{5} & \multirow[b]{2}{*}{1} & \multirow[b]{2}{*}{18} & \multirow[b]{2}{*}{25} & \multirow[b]{2}{*}{8} & \multirow[b]{2}{*}{7} & \multirow[b]{2}{*}{20} & \multirow[b]{2}{*}{23} & \multirow[b]{2}{*}{58} & \multirow[b]{2}{*}{83} \\
\hline Total & & & & & & & & & & & \\
\hline
\end{tabular}

*Only those diagnostic categories and gender/age combinations with at least one OSHA event appear in this table. 
Nevada National Security Site 2010

OSHA Data

Appendix P. Number of Workdays Lost or with Restricted Activity in Each Diagnostic Category by Gender and Age*

\begin{tabular}{|c|c|c|c|c|c|c|c|c|c|}
\hline & & \multicolumn{8}{|c|}{ Women } \\
\hline & & \multicolumn{8}{|c|}{ Age Group } \\
\hline & & \multicolumn{2}{|c|}{$16-29$} & \multicolumn{2}{|c|}{30 - 39} & \multicolumn{2}{|c|}{$40-49$} & \multicolumn{2}{|c|}{$50+$} \\
\hline & & $\begin{array}{c}\text { Days } \\
\text { Restricted }\end{array}$ & $\begin{array}{l}\text { Days } \\
\text { Lost }\end{array}$ & $\begin{array}{c}\text { Days } \\
\text { Restricted }\end{array}$ & $\begin{array}{l}\text { Days } \\
\text { Lost }\end{array}$ & $\begin{array}{c}\text { Days } \\
\text { Restricted }\end{array}$ & $\begin{array}{l}\text { Days } \\
\text { Lost }\end{array}$ & $\begin{array}{c}\text { Days } \\
\text { Restricted }\end{array}$ & $\begin{array}{c}\text { Days } \\
\text { Lost }\end{array}$ \\
\hline Diagnostic Category & ICD-9-CM Codes & & & & & & & & \\
\hline -Arthropathies & $710-719$ & 0 & 0 & 0 & 0 & 0 & 0 & 152 & 6 \\
\hline -Dorsopathies & $720-724$ & 0 & 0 & 0 & 0 & 0 & 0 & 0 & 0 \\
\hline -Rheumatism, Excluding Back & $725-729$ & 0 & 0 & 0 & 0 & 0 & 0 & 0 & $\overline{0}$ \\
\hline -Fracture - Lower Limb & $820-829$ & 0 & 0 & 0 & 0 & 0 & 0 & 0 & 0 \\
\hline -Dislocation & $830-839$ & 0 & 0 & 0 & 0 & 0 & 0 & 23 & 6 \\
\hline -Sprains \& Strains - Back & 846-847 & 0 & 0 & 0 & 0 & 0 & 0 & 0 & $\overline{0}$ \\
\hline -Sprains \& Strains - Other & $840-845,848$ & 16 & 24 & 0 & 0 & 0 & 0 & 23 & $\overline{6}$ \\
\hline -Open Wound - Lower Limb & 890-897 & 0 & 0 & 0 & 0 & 0 & 0 & 0 & 0 \\
\hline -Superficial Injury & \begin{tabular}{|c|}
$910-919$ \\
\end{tabular} & 0 & 0 & 0 & 0 & 0 & 0 & 0 & 0 \\
\hline $\begin{array}{l}\text {-Complications \& Unspecified } \\
\text { Injuries }\end{array}$ & \begin{tabular}{|c|}
$958-959$ \\
\end{tabular} & 0 & 0 & 0 & 0 & 0 & 63 & 129 & 0 \\
\hline
\end{tabular}

\begin{tabular}{|c|c|c|c|c|c|c|c|c|c|}
\hline & & \multicolumn{8}{|c|}{ Men } \\
\hline & & \multicolumn{8}{|c|}{ Age Group } \\
\hline & & \multicolumn{2}{|c|}{$16-29$} & \multicolumn{2}{|c|}{30 - 39} & \multicolumn{2}{|c|}{$40-49$} & \multicolumn{2}{|c|}{$50+$} \\
\hline & & $\begin{array}{c}\text { Days } \\
\text { Restricted }\end{array}$ & $\begin{array}{l}\text { Days } \\
\text { Lost }\end{array}$ & $\begin{array}{c}\text { Days } \\
\text { Restricted }\end{array}$ & $\begin{array}{l}\text { Days } \\
\text { Lost }\end{array}$ & $\begin{array}{c}\text { Days } \\
\text { Restricted }\end{array}$ & $\begin{array}{l}\text { Days } \\
\text { Lost }\end{array}$ & $\begin{array}{c}\text { Days } \\
\text { Restricted }\end{array}$ & $\begin{array}{l}\text { Days } \\
\text { Lost }\end{array}$ \\
\hline Diagnostic Category & ICD-9-CM Codes & & & & & & & & \\
\hline -Diseases of Ear \& Mastoid & $380-389$ & 0 & 0 & 0 & 0 & 0 & 0 & 0 & 0 \\
\hline -Lung Dis from External Agents & $500-508$ & 0 & 0 & 0 & 0 & 0 & 0 & 0 & 0 \\
\hline -Arthropathies & $710-719$ & 0 & 3 & 0 & 0 & 0 & 53 & 20 & 6 \\
\hline -Dorsopathies & $720-724$ & 7 & 0 & 2 & 59 & 19 & 106 & 12 & 0 \\
\hline -Rheumatism, Excluding Back & 725-729 & 0 & 0 & 0 & 0 & 74 & 16 & 0 & 0 \\
\hline -Symptoms & 780-789 & 0 & 0 & 0 & 0 & 0 & 0 & 0 & 0 \\
\hline -Fracture - Upper Limb & $810-819$ & 0 & 0 & 0 & 0 & 0 & 0 & 0 & 31 \\
\hline -Sprains \& Strains - Back & 846-847 & 7 & 0 & 2 & 59 & 19 & 121 & 12 & 30 \\
\hline -Sprains \& Strains - Other & $840-845,848$ & 0 & 0 & 0 & 22 & 0 & 34 & 0 & 41 \\
\hline -Intracranial Injury & $850-854$ & 0 & 0 & 0 & 0 & 0 & 0 & 0 & 0 \\
\hline -Open Wound - Head, Neck, Trunk & 870-879 & 0 & 0 & 0 & 0 & 0 & 0 & 0 & 0 \\
\hline -Open Wound - Upper Limb & 880-887 & 0 & 0 & 0 & 0 & 0 & 0 & 0 & 0 \\
\hline -Superficial Injury & $910-919$ & 0 & 0 & 0 & 0 & 0 & 0 & 20 & 6 \\
\hline -Contusion & $920-924$ & 0 & 0 & 0 & 0 & 0 & 47 & 20 & 6 \\
\hline -Burns & $940-949$ & 0 & 0 & 0 & 0 & 0 & 0 & 0 & 0 \\
\hline $\begin{array}{l}\text {-Complications \& Unspecified } \\
\text { Injuries }\end{array}$ & 958-959 & 0 & 3 & 0 & 0 & 74 & 16 & 0 & 180 \\
\hline
\end{tabular}

*OSHA events with >1 ICD-9-CM code in the same diagnostic category were counted only once. Only those diagnostic categories and gender/age combinations with at least one occurrence appear in this table. 
Nevada National Security Site 2010

OSHA Data

Appendix Q. Number of Occurrences in Each Accident Category by Gender and Age*

\begin{tabular}{|c|c|c|c|c|c|c|}
\hline & & \multicolumn{5}{|c|}{ Women } \\
\hline & & \multicolumn{4}{|c|}{ Age Group } & \multirow[b]{2}{*}{ TOTAL } \\
\hline & & $16-29$ & $30-39$ & $40-49$ & $50+$ & \\
\hline Type of Accident & E Codes & \multirow[b]{2}{*}{1} & \multirow[b]{2}{*}{1} & \multirow[b]{2}{*}{0} & \multirow[b]{2}{*}{0} & \multirow[b]{2}{*}{2} \\
\hline Motor Vehicle Traffic & E810-E819 & & & & & \\
\hline Motor Vehicle Nontraffic & E820-E825 & 0 & 0 & 0 & 0 & 0 \\
\hline Falls & E880-E888 & 0 & 0 & 1 & 4 & 5 \\
\hline Fire & E890-E899 & 0 & 0 & 0 & 0 & 0 \\
\hline Natural/Environmental Factors & E900-E909 & 0 & 0 & 0 & 0 & 0 \\
\hline Other Accidents & E916-E928 & 0 & 1 & 0 & 5 & 6 \\
\hline Homicide / Not Self-Inflicted & E960-E969 & 0 & 0 & 0 & 0 & 0 \\
\hline
\end{tabular}

\begin{tabular}{|c|c|c|c|c|c|c|c|}
\hline & & \multicolumn{5}{|c|}{ Men } & \multirow[b]{3}{*}{ TOTAL } \\
\hline & & \multicolumn{4}{|c|}{ Age Group } & \multirow[b]{2}{*}{ TOTAL } & \\
\hline & & $16-29$ & 30 - 39 & 40 - 49 & $50+$ & & \\
\hline Type of Accident & E Codes & & & & & & \\
\hline Motor Vehicle Traffic & E810-E819 & 0 & 0 & 1 & 2 & 3 & 5 \\
\hline Motor Vehicle Nontraffic & E820-E825 & 0 & 0 & 0 & 1 & 1 & 1 \\
\hline Falls & E880-E888 & 0 & 2 & 1 & 2 & 5 & 10 \\
\hline Fire & E890-E899 & 0 & 1 & 0 & 0 & 1 & 1 \\
\hline Natural/Environmental Factors & E900-E909 & 0 & 0 & 0 & 1 & 1 & 1 \\
\hline Other Accidents & E916-E928 & 4 & 3 & 9 & 7 & 23 & 29 \\
\hline Homicide / Not Self-Inflicted & E960-E969 & 0 & 0 & 0 & 2 & 2 & 2 \\
\hline
\end{tabular}

*Only those accident types and gender/age combinations with at least one occurrence appear in this table. 
Nevada National Security Site 2010

OSHA Data

Appendix R. Number of Workdays Lost or with Restricted Activity in Each Accident Category by Gender and Age*

\begin{tabular}{|c|c|c|c|c|c|c|c|c|c|}
\hline & & \multicolumn{8}{|c|}{ Women } \\
\hline & & \multicolumn{8}{|c|}{ Age Group } \\
\hline & & \multicolumn{2}{|c|}{$16-29$} & \multicolumn{2}{|c|}{$30-39$} & \multicolumn{2}{|c|}{$40-49$} & \multicolumn{2}{|c|}{$\mathbf{5 0}+$} \\
\hline & & $\begin{array}{c}\text { Days } \\
\text { Restricted }\end{array}$ & $\begin{array}{l}\text { Days } \\
\text { Lost }\end{array}$ & $\begin{array}{c}\text { Days } \\
\text { Restricted }\end{array}$ & $\begin{array}{l}\text { Days } \\
\text { Lost }\end{array}$ & $\begin{array}{c}\text { Days } \\
\text { Restricted }\end{array}$ & $\begin{array}{l}\text { Days } \\
\text { Lost }\end{array}$ & $\begin{array}{c}\text { Days } \\
\text { Restricted }\end{array}$ & $\begin{array}{l}\text { Days } \\
\text { Lost }\end{array}$ \\
\hline Type of Accident & E Codes & \multirow[b]{2}{*}{16} & \multirow[b]{2}{*}{24} & \multirow[b]{2}{*}{0} & \multirow[b]{2}{*}{0} & \multirow[b]{2}{*}{0} & \multirow[b]{2}{*}{0} & \multirow[b]{2}{*}{0} & \multirow[b]{2}{*}{0} \\
\hline Motor Vehicle Traffic & E810-E819 & & & & & & & & \\
\hline Falls & E880-E888 & 0 & 0 & 0 & 0 & 0 & 63 & 0 & 0 \\
\hline Other Accidents & E916-E928 & 0 & 0 & 0 & 0 & 0 & 0 & 152 & 6 \\
\hline
\end{tabular}

\begin{tabular}{|c|c|c|c|c|c|c|c|c|c|}
\hline & & \multicolumn{8}{|c|}{ Men } \\
\hline & & \multicolumn{8}{|c|}{ Age Group } \\
\hline & & \multicolumn{2}{|c|}{$16-29$} & \multicolumn{2}{|c|}{$30-39$} & \multicolumn{2}{|c|}{$40-49$} & \multicolumn{2}{|c|}{$50+$} \\
\hline & & $\begin{array}{c}\text { Days } \\
\text { Restricted }\end{array}$ & $\begin{array}{l}\text { Days } \\
\text { Lost }\end{array}$ & $\begin{array}{c}\text { Days } \\
\text { Restricted }\end{array}$ & $\begin{array}{l}\text { Days } \\
\text { Lost }\end{array}$ & $\begin{array}{c}\text { Days } \\
\text { Restricted }\end{array}$ & $\begin{array}{l}\text { Days } \\
\text { Lost }\end{array}$ & $\begin{array}{c}\text { Days } \\
\text { Restricted }\end{array}$ & $\begin{array}{l}\text { Days } \\
\text { Lost }\end{array}$ \\
\hline Type of Accident & E Codes & \multirow[b]{2}{*}{0} & \multirow[b]{2}{*}{0} & \multirow[b]{2}{*}{0} & \multirow[b]{2}{*}{0} & \multirow[b]{2}{*}{0} & \multirow[b]{2}{*}{10} & \multirow[b]{2}{*}{0} & \multirow[b]{2}{*}{180} \\
\hline Motor Vehicle Traffic & E810-E819 & & & & & & & & \\
\hline Motor Vehicle Nontraffic & E820-E825 & 0 & 0 & 0 & 0 & 0 & 0 & 20 & 6 \\
\hline Falls & E880-E888 & 0 & 0 & 0 & 22 & 0 & 18 & 0 & 31 \\
\hline Fire & E890-E899 & 0 & 0 & 0 & 0 & 0 & 0 & 0 & 0 \\
\hline Natural/Environmental Factors & E900-E909 & 0 & 0 & 0 & 0 & 0 & 0 & 0 & 0 \\
\hline Other Accidents & E916-E928 & 7 & 3 & 2 & 59 & 93 & 190 & 12 & 41 \\
\hline Homicide / Not Self-Inflicted & E960-E969 & 0 & 0 & 0 & 0 & 0 & 0 & 0 & 30 \\
\hline
\end{tabular}

*OSHA events with $>1 \mathrm{E}$ code in the same accident type were counted only once. Only those accident types and gender/age combinations with at least one occurrence appear in this table. 
Nevada National Security Site 2010

OSHA Data

Appendix S. Number of Diagnoses in Each Diagnostic Category by Gender and Job Category*

\begin{tabular}{|c|c|c|c|c|c|c|c|}
\hline & & \multicolumn{6}{|c|}{ Women } \\
\hline & & \multicolumn{5}{|c|}{ Job Category } & \multirow[b]{2}{*}{ TOTAL } \\
\hline & & Professional & \begin{tabular}{|} 
Administrative \\
Support
\end{tabular} & Technical Support & Service & Crafts & \\
\hline Diagnostic Category & ICD-9-CM Code & & & & & & \\
\hline $\begin{array}{l}\text { MUSCULOSKELETAL } \\
\text { \& CONNECTIVE } \\
\text { TISSUE }\end{array}$ & $710-739$ & 3 & 0 & 6 & 0 & 0 & 9 \\
\hline -Arthropathies & $710-719$ & 2 & 0 & 4 & 0 & 0 & 6 \\
\hline -Dorsopathies & $720-724$ & 1 & 0 & 1 & 0 & 0 & 2 \\
\hline $\begin{array}{l}\text {-Rheumatism, Excluding } \\
\text { Back }\end{array}$ & $725-729$ & 0 & 0 & 1 & 0 & 0 & 1 \\
\hline INJURY \& POISONING & $800-999$ & 4 & 4 & 4 & 3 & 1 & 16 \\
\hline -Fracture - Lower Limb & $820-829$ & 0 & 2 & 0 & 0 & 0 & 2 \\
\hline -Dislocation & 830-839 & 1 & 0 & 0 & 0 & 0 & 1 \\
\hline -Sprains \& Strains - Back & 846-847 & 1 & 0 & 1 & 0 & 0 & 2 \\
\hline $\begin{array}{l}\text {-Sprains \& Strains - } \\
\text { Other }\end{array}$ & $840-845,848$ & 1 & 0 & 2 & 0 & 0 & 3 \\
\hline $\begin{array}{l}\text {-Open Wound - Lower } \\
\text { Limb }\end{array}$ & $890-897$ & 1 & 0 & 0 & 0 & 0 & 1 \\
\hline -Superficial Injury & $910-919$ & 0 & 1 & 0 & 0 & 0 & 1 \\
\hline $\begin{array}{l}\text {-Complications \& } \\
\text { Unspecified Injuries }\end{array}$ & \begin{tabular}{|l|}
$958-959$ \\
\end{tabular} & 0 & 1 & 1 & 3 & 1 & 6 \\
\hline
\end{tabular}

\begin{tabular}{|c|c|c|c|c|c|c|}
\hline & \multicolumn{6}{|c|}{ Women } \\
\hline & \multicolumn{5}{|c|}{ Job Category } & \multirow[b]{2}{*}{ TOTAL } \\
\hline & Professional & $\begin{array}{c}\text { Administrative } \\
\text { Support }\end{array}$ & Technical Support & Service & Crafts & \\
\hline Diagnostic Category & \multirow[b]{2}{*}{7} & \multirow[b]{2}{*}{4} & \multirow[b]{2}{*}{10} & \multirow[b]{2}{*}{3} & \multirow[b]{2}{*}{1} & \multirow[b]{2}{*}{25} \\
\hline Total & & & & & & \\
\hline
\end{tabular}

\begin{tabular}{|c|c|c|c|c|c|c|}
\hline & & \multicolumn{5}{|c|}{ Men } \\
\hline & & \multicolumn{4}{|c|}{ Job Category } & \multirow[b]{2}{*}{ TOTAL } \\
\hline & & Professional & Technical Support & Service & Crafts & \\
\hline Diagnostic Category & ICD-9-CM Code & & & & & \\
\hline $\begin{array}{l}\text { NERVOUS SYSTEM } \\
\text { (NS) \& SENSE ORGANS }\end{array}$ & 320-389 & 0 & 0 & 0 & 1 & 1 \\
\hline $\begin{array}{l}\text {-Diseases of } \\
\text { Ear \& Mastoid }\end{array}$ & 380-389 & 0 & 0 & 0 & 1 & 1 \\
\hline $\begin{array}{l}\text { RESPIRATORY } \\
\text { SYSTEM }\end{array}$ & $460-519$ & 0 & 1 & 0 & 0 & 1 \\
\hline $\begin{array}{l}\text {-Lung Dis from External } \\
\text { Agents }\end{array}$ & $500-508$ & 0 & 1 & 0 & 0 & 1 \\
\hline $\begin{array}{l}\text { MUSCULOSKELETAL } \\
\text { \& CONNECTIVE } \\
\text { TISSUE }\end{array}$ & \begin{tabular}{|l|}
$710-739$ \\
\end{tabular} & 2 & 9 & 0 & 5 & 16 \\
\hline
\end{tabular}

(Continued)

*Only those diagnostic categories and gender/job category combinations with at least one occurrence appear in this table. 
Nevada National Security Site 2010

OSHA Data

Appendix S. Number of Diagnoses in Each Diagnostic Category by Gender and Job Category*

\begin{tabular}{|c|c|c|c|c|c|c|}
\hline & & \multicolumn{5}{|c|}{ Men } \\
\hline & & \multicolumn{4}{|c|}{ Job Category } & \multirow[b]{2}{*}{ TOTAL } \\
\hline & & Professional & Technical Support & Service & Crafts & \\
\hline Diagnostic Category & ICD-9-CM Code & & & & & \\
\hline -Arthropathies & $710-719$ & 1 & 5 & 0 & 2 & 8 \\
\hline -Dorsopathies & $720-724$ & 1 & 4 & 0 & 1 & 6 \\
\hline $\begin{array}{l}\text {-Rheumatism, Excluding } \\
\text { Back }\end{array}$ & 725-729 & 0 & 0 & 0 & 2 & 2 \\
\hline $\begin{array}{l}\text { SYMPTOMS, } \\
\text { SIGNS, \& ILL-DEFINED } \\
\text { CONDITIONS }\end{array}$ & $780-799$ & 1 & 0 & 0 & 0 & 1 \\
\hline -Symptoms & $780-789$ & 1 & 0 & 0 & 0 & 1 \\
\hline INJURY \& POISONING & 800-999 & 9 & 18 & 2 & 10 & 39 \\
\hline -Fracture - Upper Limb & 810-819 & 1 & 0 & 0 & 1 & 2 \\
\hline -Sprains \& Strains - Back & 846-847 & 1 & 5 & 0 & 3 & 9 \\
\hline $\begin{array}{l}\text {-Sprains \& Strains - } \\
\text { Other }\end{array}$ & $840-845,848$ & 2 & 6 & 0 & 2 & 10 \\
\hline -Intracranial Injury & $850-854$ & 0 & 0 & 1 & 0 & 1 \\
\hline $\begin{array}{l}\text {-Open Wound - Head, } \\
\text { Neck, Trunk }\end{array}$ & $870-879$ & 1 & 2 & 1 & 0 & 4 \\
\hline $\begin{array}{l}\text {-Open Wound - Upper } \\
\text { Limb }\end{array}$ & 880-887 & 3 & 0 & 0 & 0 & 3 \\
\hline -Superficial Injury & 910-919 & 0 & 2 & 0 & 0 & 2 \\
\hline -Contusion & $920-924$ & 0 & 1 & 0 & 1 & 2 \\
\hline -Burns & 940-949 & 1 & 0 & 0 & 0 & 1 \\
\hline $\begin{array}{l}\text {-Complications \& } \\
\text { Unspecified Injuries }\end{array}$ & 958-959 & 0 & 2 & 0 & 3 & 5 \\
\hline
\end{tabular}

\begin{tabular}{|l|r|r|r|r|r|}
\hline & \multicolumn{4}{|c|}{ Men } & \\
\cline { 2 - 5 } & \multicolumn{3}{|c|}{ Job Category } & \\
\cline { 2 - 6 } & Professional & Technical Support & Service & Crafts & TOTAL \\
\hline Diagnostic Category & & 28 & 2 & 16 & 58 \\
\hline Total & 12 & & & & \\
\hline
\end{tabular}

*Only those diagnostic categories and gender/job category combinations with at least one occurrence appear in this table. 
Nevada National Security Site 2010

OSHA Data

Appendix T. Number of Workdays Lost or with Restricted Activity in Each Diagnostic Category by Gender and Job Category*

\begin{tabular}{|c|c|c|c|c|c|}
\hline & & \multicolumn{4}{|c|}{ Women } \\
\hline & & \multicolumn{4}{|c|}{ Job Category } \\
\hline & & \multicolumn{2}{|c|}{ Professional } & \multicolumn{2}{|c|}{$\begin{array}{l}\text { Administrative } \\
\text { Support }\end{array}$} \\
\hline & & $\begin{array}{c}\text { Days } \\
\text { Restricted }\end{array}$ & Days Lost & $\begin{array}{c}\text { Days } \\
\text { Restricted }\end{array}$ & Days Lost \\
\hline Diagnostic Category & ICD-9-CM Codes & \multirow[b]{2}{*}{23} & \multirow[b]{2}{*}{6} & \multirow[b]{2}{*}{0} & \multirow[b]{2}{*}{0} \\
\hline -Arthropathies & $710-719$ & & & & \\
\hline -Dorsopathies & $720-724$ & 0 & 0 & 0 & 0 \\
\hline -Rheumatism, Excluding Back & 725-729 & 0 & 0 & 0 & 0 \\
\hline -Fracture - Lower Limb & 820-829 & 0 & 0 & 0 & 0 \\
\hline -Dislocation & 830-839 & 23 & 6 & 0 & 0 \\
\hline -Sprains \& Strains - Back & 846-847 & 0 & 0 & 0 & 0 \\
\hline -Sprains \& Strains - Other & $840-845,848$ & 23 & 6 & 0 & $\overline{0}$ \\
\hline -Open Wound - Lower Limb & $890-897$ & 0 & 0 & 0 & 0 \\
\hline -Superficial Injury & 910-919 & 0 & 0 & 0 & 0 \\
\hline $\begin{array}{l}\text {-Complications \& Unspecified } \\
\text { Injuries }\end{array}$ & 958-959 & 0 & 0 & 0 & 0 \\
\hline
\end{tabular}

\begin{tabular}{|c|c|c|c|c|c|c|c|}
\hline & & \multicolumn{6}{|c|}{ Women } \\
\hline & & \multicolumn{6}{|c|}{ Job Category } \\
\hline & & \multicolumn{2}{|c|}{ Technical Support } & \multicolumn{2}{|c|}{ Service } & \multicolumn{2}{|c|}{ Crafts } \\
\hline & & $\begin{array}{c}\text { Days } \\
\text { Restricted }\end{array}$ & Days Lost & $\begin{array}{c}\text { Days } \\
\text { Restricted }\end{array}$ & Days Lost & $\begin{array}{c}\text { Days } \\
\text { Restricted }\end{array}$ & Days Lost \\
\hline Diagnostic Category & ICD-9-CM Codes & & & & & & \\
\hline -Arthropathies & $710-719$ & 129 & 0 & 0 & 0 & 0 & 0 \\
\hline -Dorsopathies & $720-724$ & 0 & 0 & 0 & 0 & 0 & 0 \\
\hline -Rheumatism, Excluding Back & 725-729 & 0 & 0 & 0 & 0 & 0 & 0 \\
\hline -Fracture - Lower Limb & $820-829$ & 0 & 0 & 0 & 0 & 0 & 0 \\
\hline -Dislocation & 830-839 & 0 & 0 & 0 & 0 & 0 & 0 \\
\hline -Sprains \& Strains - Back & $846-847$ & 0 & 0 & 0 & 0 & 0 & 0 \\
\hline -Sprains \& Strains - Other & $840-845,848$ & 16 & 24 & 0 & 0 & 0 & 0 \\
\hline -Open Wound - Lower Limb & $890-897$ & 0 & 0 & 0 & 0 & 0 & 0 \\
\hline -Superficial Injury & 910-919 & 0 & 0 & 0 & 0 & 0 & 0 \\
\hline $\begin{array}{l}\text {-Complications \& Unspecified } \\
\text { Injuries }\end{array}$ & 958-959 & 129 & 0 & 0 & 0 & 0 & 63 \\
\hline
\end{tabular}

*OSHA events with >1 ICD-9-CM code in the same diagnostic category were counted only once. Only those diagnostic categories and gender/job category combinations with at least one occurrence appear in this table. 
Nevada National Security Site 2010

OSHA Data

Appendix T. Number of Workdays Lost or with Restricted Activity in Each Diagnostic Category by Gender and Job Category*

\begin{tabular}{|c|c|c|c|c|c|c|c|c|c|}
\hline & & \multicolumn{8}{|c|}{ Men } \\
\hline & & \multicolumn{8}{|c|}{ Job Category } \\
\hline & & \multicolumn{2}{|c|}{ Professional } & \multicolumn{2}{|c|}{ Technical Support } & \multicolumn{2}{|c|}{ Service } & \multicolumn{2}{|c|}{ Crafts } \\
\hline & & $\begin{array}{c}\text { Days } \\
\text { Restricted }\end{array}$ & $\begin{array}{l}\text { Days } \\
\text { Lost }\end{array}$ & \begin{tabular}{c|} 
Days \\
Restricted
\end{tabular} & $\begin{array}{l}\text { Days } \\
\text { Lost }\end{array}$ & $\begin{array}{c}\text { Days } \\
\text { Restricted }\end{array}$ & $\begin{array}{l}\text { Days } \\
\text { Lost }\end{array}$ & $\begin{array}{c}\text { Days } \\
\text { Restricted }\end{array}$ & $\begin{array}{l}\text { Days } \\
\text { Lost }\end{array}$ \\
\hline Diagnostic Category & ICD-9-CM Codes & & & & & & & & \\
\hline -Diseases of Ear \& Mastoid & 380-389 & 0 & 0 & 0 & 0 & 0 & 0 & 0 & 0 \\
\hline -Lung Dis from External Agents & $500-508$ & 0 & 0 & 0 & 0 & 0 & 0 & 0 & 0 \\
\hline -Arthropathies & $710-719$ & 0 & 0 & 20 & 15 & 0 & 0 & 0 & 47 \\
\hline -Dorsopathies & $720-724$ & 0 & 0 & 21 & 59 & 0 & 0 & 19 & 106 \\
\hline -Rheumatism, Excluding Back & $725-729$ & 0 & 0 & 0 & 0 & 0 & 0 & 74 & 16 \\
\hline -Symptoms & 780-789 & 0 & 0 & 0 & 0 & 0 & 0 & 0 & 0 \\
\hline -Fracture - Upper Limb & 810-819 & 0 & 31 & 0 & 0 & 0 & 0 & 0 & 0 \\
\hline -Sprains \& Strains - Back & $846-847$ & 0 & 0 & 21 & 74 & 0 & 0 & 19 & 136 \\
\hline -Sprains \& Strains - Other & $840-845,848$ & 0 & 0 & 0 & 56 & 0 & 0 & 0 & 41 \\
\hline -Intracranial Injury & $850-854$ & 0 & 0 & 0 & 0 & 0 & 0 & 0 & $\overline{0}$ \\
\hline -Open Wound - Head, Neck, Trunk & 870-879 & 0 & 0 & 0 & 0 & 0 & 0 & 0 & 0 \\
\hline -Open Wound - Upper Limb & $880-887$ & 0 & 0 & 0 & 0 & 0 & 0 & 0 & 0 \\
\hline -Superficial Injury & $910-919$ & 0 & 0 & 20 & 6 & 0 & 0 & 0 & 0 \\
\hline -Contusion & $920-924$ & 0 & 0 & 20 & 6 & 0 & 0 & 0 & 47 \\
\hline -Burns & $940-949$ & 0 & 0 & 0 & 0 & 0 & 0 & 0 & 0 \\
\hline $\begin{array}{l}\text {-Complications \& Unspecified } \\
\text { Injuries }\end{array}$ & 958-959 & 0 & 0 & 0 & 3 & 0 & 0 & 74 & 196 \\
\hline
\end{tabular}

*OSHA events with >1 ICD-9-CM code in the same diagnostic category were counted only once. Only those diagnostic categories and gender/job category combinations with at least one occurrence appear in this table. 
Nevada National Security Site 2010

OSHA Data

Appendix U. Number of Occurrences in Each Accident Category by Gender and Job Category*

\begin{tabular}{|c|c|c|c|c|c|c|c|}
\hline & & \multicolumn{6}{|c|}{ Women } \\
\hline & & \multicolumn{5}{|c|}{ Job Category } & \multirow[b]{2}{*}{ TOTAL } \\
\hline & & Professional & $\begin{array}{c}\text { Administrative } \\
\text { Support }\end{array}$ & \begin{tabular}{|l|} 
Technical \\
Support
\end{tabular} & Service & Crafts & \\
\hline Type of Accident & E CODES & \multirow[b]{2}{*}{1} & \multirow[b]{2}{*}{0} & \multirow[b]{2}{*}{1} & \multirow[b]{2}{*}{0} & \multirow[b]{2}{*}{0} & \multirow[b]{2}{*}{2} \\
\hline Motor Vehicle Traffic & E810-E819 & & & & & & \\
\hline Falls & E880-E888 & 0 & 2 & 0 & 2 & 1 & 5 \\
\hline Other Accidents & \begin{tabular}{|l|} 
E916-E928 \\
\end{tabular} & 2 & 0 & 4 & 0 & 0 & 6 \\
\hline
\end{tabular}

\begin{tabular}{|c|c|c|c|c|c|c|}
\hline & & \multicolumn{5}{|c|}{ Men } \\
\hline & & \multicolumn{4}{|c|}{ Job Category } & \multirow[b]{2}{*}{ TOTAL } \\
\hline & & Professional & $\begin{array}{l}\text { Technical } \\
\text { Support }\end{array}$ & Service & Crafts & \\
\hline Type of Accident & E CODES & \multirow[b]{2}{*}{0} & \multirow[b]{2}{*}{2} & \multirow[b]{2}{*}{0} & \multirow[b]{2}{*}{1} & \multirow[b]{2}{*}{3} \\
\hline Motor Vehicle Traffic & E810-E819 & & & & & \\
\hline Motor Vehicle Nontraffic & E820-E825 & 0 & 1 & 0 & 0 & 1 \\
\hline Falls & E880-E888 & 1 & 4 & 0 & 0 & 5 \\
\hline Fire & E890-E899 & 1 & 0 & 0 & 0 & 1 \\
\hline Natural/Environmental Factors & E900-E909 & 1 & 0 & 0 & 0 & 1 \\
\hline Other Accidents & E916-E928 & 6 & 9 & 1 & 7 & 23 \\
\hline Homicide / Not Self-Inflicted & E960-E969 & 0 & 0 & 0 & 2 & 2 \\
\hline
\end{tabular}

*Only those accident types and gender/job category combinations with at least one occurrence appear in this table. 
Nevada National Security Site 2010

OSHA Data

Appendix V. Number of Workdays Lost or with Restricted Activity in Each Accident Category by Gender and Job Category*

\begin{tabular}{|c|c|c|c|c|c|}
\hline & & \multicolumn{4}{|c|}{ Women } \\
\hline & & \multicolumn{4}{|c|}{ Job Category } \\
\hline & & \multicolumn{2}{|c|}{ Professional } & \multicolumn{2}{|c|}{$\begin{array}{l}\text { Administrative } \\
\text { Support }\end{array}$} \\
\hline & & $\begin{array}{c}\text { Days } \\
\text { Restricted }\end{array}$ & Days Lost & $\begin{array}{c}\text { Days } \\
\text { Restricted }\end{array}$ & Days Lost \\
\hline Type of Accident & E Codes & \multirow[b]{2}{*}{0} & \multirow[b]{2}{*}{0} & \multirow[b]{2}{*}{0} & \multirow[b]{2}{*}{0} \\
\hline Motor Vehicle Traffic & E810-E819 & & & & \\
\hline Falls & E880-E888 & 0 & 0 & 0 & 0 \\
\hline Other Accidents & E916-E928 & 23 & 6 & 0 & 0 \\
\hline
\end{tabular}

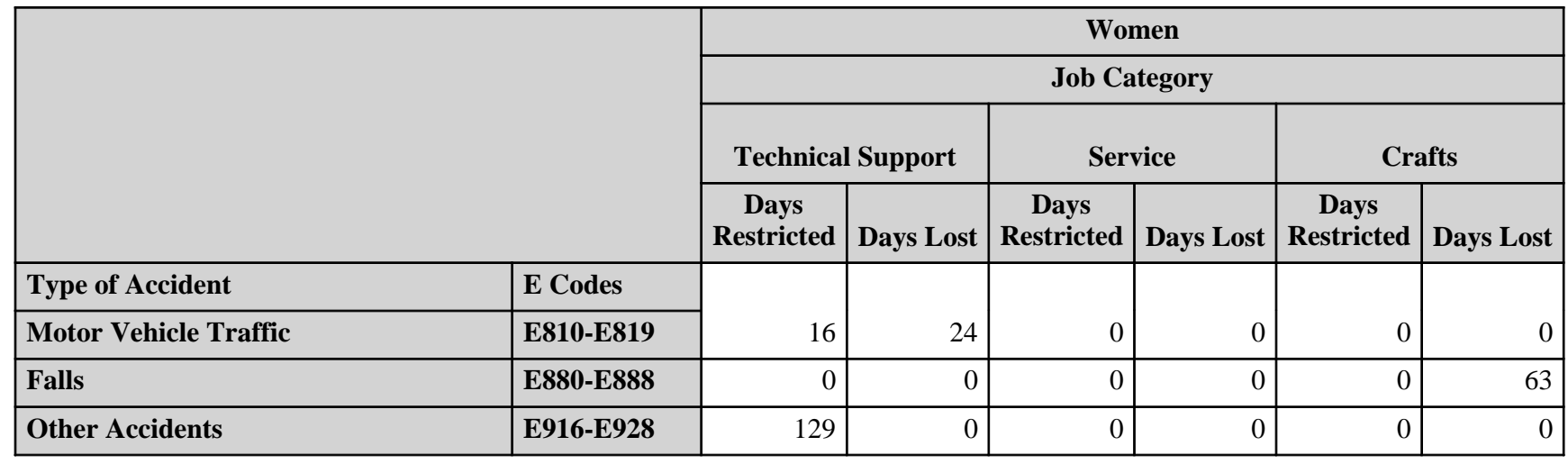

\begin{tabular}{|c|c|c|c|c|c|c|c|c|c|}
\hline & & \multicolumn{8}{|c|}{ Men } \\
\hline & & \multicolumn{8}{|c|}{ Job Category } \\
\hline & & \multicolumn{2}{|c|}{ Professional } & \multicolumn{2}{|c|}{ Technical Support } & \multicolumn{2}{|c|}{ Service } & \multicolumn{2}{|c|}{ Crafts } \\
\hline & & $\begin{array}{c}\text { Days } \\
\text { Restricted }\end{array}$ & $\begin{array}{l}\text { Days } \\
\text { Lost }\end{array}$ & $\begin{array}{c}\text { Days } \\
\text { Restricted }\end{array}$ & $\begin{array}{l}\text { Days } \\
\text { Lost }\end{array}$ & $\begin{array}{c}\text { Days } \\
\text { Restricted }\end{array}$ & $\begin{array}{l}\text { Days } \\
\text { Lost }\end{array}$ & $\begin{array}{c}\text { Days } \\
\text { Restricted }\end{array}$ & $\begin{array}{l}\text { Days } \\
\text { Lost }\end{array}$ \\
\hline Type of Accident & E Codes & \multirow[b]{2}{*}{0} & \multirow[b]{2}{*}{0} & \multirow[b]{2}{*}{0} & \multirow[b]{2}{*}{10} & \multirow[b]{2}{*}{0} & \multirow[b]{2}{*}{0} & \multirow[b]{2}{*}{0} & \multirow[b]{2}{*}{180} \\
\hline Motor Vehicle Traffic & E810-E819 & & & & & & & & \\
\hline Motor Vehicle Nontraffic & E820-E825 & 0 & 0 & 20 & 6 & 0 & 0 & 0 & 0 \\
\hline Falls & E880-E888 & 0 & 31 & 0 & 40 & 0 & 0 & 0 & 0 \\
\hline Fire & E890-E899 & 0 & 0 & 0 & 0 & 0 & 0 & 0 & 0 \\
\hline Natural/Environmental Factors & E900-E909 & 0 & 0 & 0 & 0 & 0 & 0 & 0 & 0 \\
\hline Other Accidents & E916-E928 & 0 & 0 & 21 & 83 & 0 & 0 & 93 & 210 \\
\hline Homicide / Not Self-Inflicted & E960-E969 & 0 & 0 & 0 & 0 & 0 & 0 & 0 & 30 \\
\hline
\end{tabular}

*OSHA events with $>1 \mathrm{E}$ code in the same accident type were counted only once. Only those accident types and gender/job category combinations with at least one occurrence appear in this table. 
Nevada National Security Site 2010

OSHA Data

Appendix W. Age-Adjusted OSHA Illness and Injury Rates by Diagnostic Category*

Part 1. Men

\begin{tabular}{|c|c|c|c|c|c|}
\hline & & $\begin{array}{l}\text { Number of } \\
\text { Diagnoses }\end{array}$ & $\begin{array}{l}\text { Age-Adjusted } \\
\text { Rate per } 1,000 * *\end{array}$ & $\begin{array}{c}\text { Lower } 95 \% \\
\text { Confidence } \\
\text { Limit per } 1,000\end{array}$ & $\begin{array}{c}\text { Upper } 95 \% \\
\text { Confidence } \\
\text { Limit per 1,000 }\end{array}$ \\
\hline Diagnostic Category & ICD-9-CM Code & & & & \\
\hline NERVOUS SYSTEM (NS) \& SENSE ORGANS & 320-389 & 1 & 0.2 & 0.0 & 1.3 \\
\hline -Diseases of Ear \& Mastoid & 380-389 & 1 & 0.2 & 0.0 & 1.3 \\
\hline RESPIRATORY SYSTEM & $460-519$ & 1 & 0.4 & 0.0 & 2.5 \\
\hline -Lung Dis from External Agents & $500-508$ & 1 & 0.4 & 0.0 & 2.5 \\
\hline $\begin{array}{l}\text { MUSCULOSKELETAL \& CONNECTIVE } \\
\text { TISSUE }\end{array}$ & $710-739$ & 16 & 8.3 & 4.6 & 15.0 \\
\hline -Arthropathies & 710-719 & 8 & 4.0 & 1.7 & 9.4 \\
\hline -Dorsopathies & $720-724$ & 6 & 3.6 & 1.4 & 9.3 \\
\hline -Rheumatism, Excluding Back & 725-729 & 2 & 0.7 & 0.2 & 2.8 \\
\hline $\begin{array}{l}\text { SYMPTOMS, SIGNS, \& ILL-DEFINED } \\
\text { CONDITIONS }\end{array}$ & \begin{tabular}{|c|}
$780-799$ \\
\end{tabular} & 1 & 0.2 & 0.0 & 1.2 \\
\hline -Symptoms & $780-789$ & 1 & 0.2 & 0.0 & 1.2 \\
\hline INJURY \& POISONING & $800-999$ & 39 & 14.4 & 9.7 & 21.4 \\
\hline -Fracture - Upper Limb & 810-819 & 2 & 0.5 & 0.1 & 2.3 \\
\hline -Sprains \& Strains - Back & 846-847 & 9 & 4.3 & 1.9 & 9.7 \\
\hline -Sprains \& Strains - Other & $840-845,848$ & 10 & 2.9 & 1.5 & 5.7 \\
\hline -Open Wound - Head, Neck, Trunk & 870-879 & 4 & 1.0 & 0.3 & 3.1 \\
\hline -Open Wound - Upper Limb & 880-887 & 3 & 2.0 & 0.5 & 7.0 \\
\hline -Superficial Injury & $910-919$ & 2 & 0.3 & 0.1 & 1.4 \\
\hline -Contusion & \begin{tabular}{|l|}
$920-924$ \\
\end{tabular} & 2 & 0.5 & 0.1 & 2.3 \\
\hline -Burns & \begin{tabular}{|c|}
$940-949$ \\
\end{tabular} & 1 & 0.5 & 0.1 & 3.5 \\
\hline -Complications of Surgical/Medical Care & 996-999 & 6 & 2.3 & 0.8 & 6.7 \\
\hline Total & & 58 & 23.4 & 17.0 & 32.3 \\
\hline
\end{tabular}

* Only those diagnostic categories with at least one occurrence appear in this table.

**Standardized to age distribution of 2000 U.S. population. 
Nevada National Security Site 2010

OSHA Data

Appendix W. Age-Adjusted OSHA Illness and Injury Rates by Diagnostic Category*

Part 2. Women

\begin{tabular}{|c|c|c|c|c|c|}
\hline & & $\begin{array}{l}\text { Number of } \\
\text { Diagnoses }\end{array}$ & $\begin{array}{c}\text { Age-Adjusted } \\
\text { Rate per } 1,000 * *\end{array}$ & $\begin{array}{c}\text { Lower } 95 \% \\
\text { Confidence } \\
\text { Limit per } 1,000\end{array}$ & $\begin{array}{c}\text { Upper } 95 \% \\
\text { Confidence } \\
\text { Limit per 1,000 }\end{array}$ \\
\hline Diagnostic Category & ICD-9-CM Code & & & & \\
\hline $\begin{array}{l}\text { MUSCULOSKELETAL \& CONNECTIVE } \\
\text { TISSUE }\end{array}$ & $710-739$ & 9 & 9.1 & 4.4 & 19.0 \\
\hline -Arthropathies & $710-719$ & 6 & 5.4 & 2.2 & 13.5 \\
\hline -Dorsopathies & $720-724$ & 2 & 2.1 & 0.4 & 10.0 \\
\hline -Rheumatism, Excluding Back & 725-729 & 1 & 1.6 & 0.2 & 11.3 \\
\hline INJURY \& POISONING & $800-999$ & 16 & 12.3 & 6.7 & 22.7 \\
\hline -Fracture - Lower Limb & 820-829 & 2 & 1.2 & 0.3 & 5.0 \\
\hline -Dislocation & $830-839$ & 1 & 0.5 & 0.1 & 3.5 \\
\hline -Sprains \& Strains - Back & 846-847 & 2 & 2.1 & 0.4 & 10.0 \\
\hline -Sprains \& Strains - Other & $840-845,848$ & 3 & 3.7 & 0.8 & 16.3 \\
\hline -Open Wound - Lower Limb & 890-897 & 1 & 0.5 & 0.1 & 3.5 \\
\hline -Superficial Injury & $910-919$ & 1 & 0.6 & 0.1 & 4.4 \\
\hline -Complications of Surgical/Medical Care & 996-999 & 6 & 3.7 & 1.6 & 8.4 \\
\hline Total & & 25 & 21.4 & 13.4 & 34.3 \\
\hline
\end{tabular}

* Only those diagnostic categories with at least one occurrence appear in this table.

**Standardized to age distribution of 2000 U.S. population. 
Nevada National Security Site 2010

OSHA Data

Appendix W. Age-Adjusted OSHA Illness and Injury Rates by Diagnostic Category*

Part 3. Men and Women

\begin{tabular}{|c|c|c|c|c|c|}
\hline & & $\begin{array}{l}\text { Number of } \\
\text { Diagnoses }\end{array}$ & $\begin{array}{c}\text { Age-Adjusted } \\
\text { Rate per } 1,000 * *\end{array}$ & $\begin{array}{c}\text { Lower } 95 \% \\
\text { Confidence } \\
\text { Limit per } 1,000\end{array}$ & $\begin{array}{c}\text { Upper 95\% } \\
\text { Confidence } \\
\text { Limit per 1,000 }\end{array}$ \\
\hline Diagnostic Category & ICD-9-CM Code & \multirow[b]{2}{*}{1} & \multirow[b]{2}{*}{0.1} & \multirow[b]{2}{*}{0.0} & \multirow[b]{2}{*}{1.0} \\
\hline NERVOUS SYSTEM (NS) \& SENSE ORGANS & 320-389 & & & & \\
\hline -Diseases of Ear \& Mastoid & 380-389 & 1 & 0.1 & 0.0 & 1.0 \\
\hline RESPIRATORY SYSTEM & $460-519$ & 1 & 0.3 & 0.0 & 1.8 \\
\hline -Lung Dis from External Agents & $500-508$ & 1 & 0.3 & 0.0 & 1.8 \\
\hline $\begin{array}{l}\text { MUSCULOSKELETAL \& CONNECTIVE } \\
\text { TISSUE }\end{array}$ & $710-739$ & 25 & 8.1 & 5.1 & 13.0 \\
\hline -Arthropathies & $710-719$ & 14 & 4.2 & 2.2 & 7.9 \\
\hline -Dorsopathies & $720-724$ & 8 & 3.1 & 1.4 & 7.0 \\
\hline -Rheumatism, Excluding Back & $725-729$ & 3 & 0.9 & 0.3 & 2.8 \\
\hline $\begin{array}{l}\text { SYMPTOMS, SIGNS, \& ILL-DEFINED } \\
\text { CONDITIONS }\end{array}$ & $780-799$ & 1 & 0.1 & 0.0 & 0.9 \\
\hline -Symptoms & $780-789$ & 1 & 0.1 & 0.0 & 0.9 \\
\hline INJURY \& POISONING & $800-999$ & 55 & 13.7 & 9.9 & 19.1 \\
\hline -Fracture - Upper Limb & 810-819 & 2 & 0.4 & 0.1 & 1.7 \\
\hline -Fracture - Lower Limb & $820-829$ & 2 & 0.3 & 0.1 & 1.1 \\
\hline -Dislocation & $830-839$ & 1 & 0.1 & 0.0 & 0.9 \\
\hline -Sprains \& Strains - Back & 846-847 & 11 & 3.6 & 1.8 & 7.4 \\
\hline -Sprains \& Strains - Other & $840-845,848$ & 13 & 3.2 & 1.6 & 6.3 \\
\hline -Open Wound - Head, Neck, Trunk & $870-879$ & 4 & 0.8 & 0.2 & 2.3 \\
\hline -Open Wound - Upper Limb & 880-887 & 3 & 1.4 & 0.4 & 4.9 \\
\hline -Open Wound - Lower Limb & $890-897$ & 1 & 0.1 & 0.0 & 0.9 \\
\hline -Superficial Injury & $910-919$ & 3 & 0.4 & 0.1 & 1.2 \\
\hline -Contusion & $920-924$ & 2 & 0.4 & 0.1 & 1.7 \\
\hline -Burns & $940-949$ & 1 & 0.4 & 0.1 & 2.6 \\
\hline -Complications of Surgical/Medical Care & 996-999 & 12 & 2.6 & 1.3 & 5.4 \\
\hline Total & & 83 & 22.4 & 17.1 & 29.2 \\
\hline
\end{tabular}

* Only those diagnostic categories with at least one occurrence appear in this table.

**Standardized to age distribution of 2000 U.S. population. 